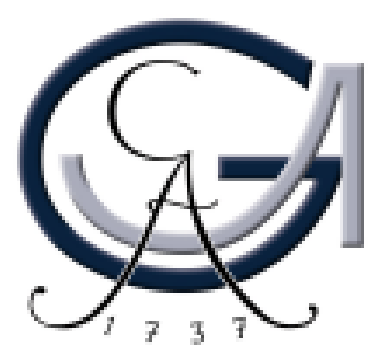

Regulation and communication between the NDR kinase COT1, the MAK2 MAP kinase cascade and the Striatin complex in Neurospora crassa

\author{
Dissertation \\ for the award of the degree \\ "Doctor rerum naturalium" \\ Division of Mathematics and Natural Sciences \\ of the Georg-August-University Göttingen
}

submitted by

Anne Dettmann

from Neubrandenburg

Göttingen June 2011 

"Es verwirren uns nicht die Dinge, die wir nicht wissen, sondern die wir wissen und die nicht so sind." 
Member of the Thesis Committee: PD Dr. Stephan Seiler (Reviewer)

Department of Molecular Microbiology and Genetics, Institute of Microbiology and Genetics Georg-August-University of Göttingen

Member of the Thesis Committee: Prof. Dr. Stefanie Pöggeler (Reviewer)

Department of Genetics of Eukaryotic Cells, Institute of Microbiology and Genetics Georg-August-University of Göttingen

Member of the Thesis Committee: Prof. Dr. Reinhard Jahn

Max Planck Institute for Biophysical Chemistry, Karl Friedrich Bonhoeffer Institute Göttingen 
I hereby confirm that this thesis has been written independently and with no other sources and aids than quoted.

Göttingen, 30.06.2011

Anne Dettmann 
Parts of this work have been or will be published:

\section{Script in preparation:}

Sabine Maerz, Anne Dettmann, and Stephan Seiler. Hydrophobic motif phosphorylation of the NDR kinase COT1 by the germinal centre kinase POD6 coordinates activity and localization of COT1 in Neurospora crassa.

Anne Dettmann, Sabine Maerz, Timo Schürg, Fleißner, A., and Stephan Seiler. HYM1/MO25 functions as a dual scaffold that connects the NDR kinase COT1 with the MAK2 MAP kinase pathway in Neurospora crassa.

Sandra Bloemendal, Yasmine Bernhards, Kathrin Bartho, Anne Dettmann, Ines Teichert, Stephan Seiler, Dirk Andreas Wolters, Stefanie Pöggeler and Ulrich Kück. A homologue of the human STRIPAK complex controls fungal sexual development.

\section{Publication:}

Maerz, S., Dettmann, A., Ziv, C., Liu, Y., Valerius, O., Yarden, O. \& Seiler, S., (2009) Two NDR kinase - MOB complexes function as distinct modules during septum formation and tip extension in Neurospora crassa. Mol. Microbiol. 



\section{Table of contents}

1. Summary .1

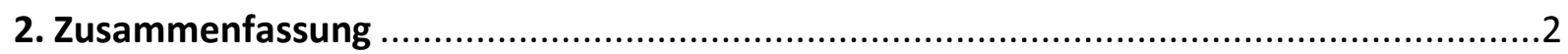

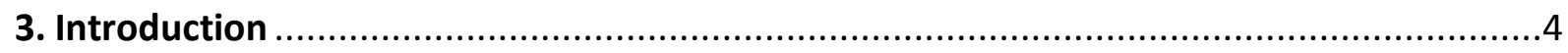

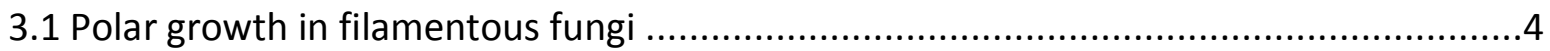

3.2 General signaling cascades that regulate polar growth (NDR, MAPK) ..........................5

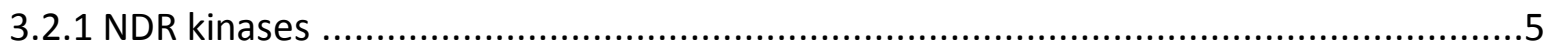

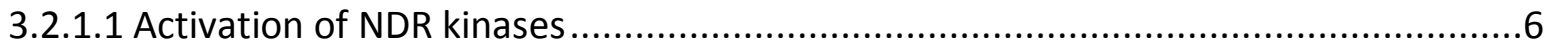

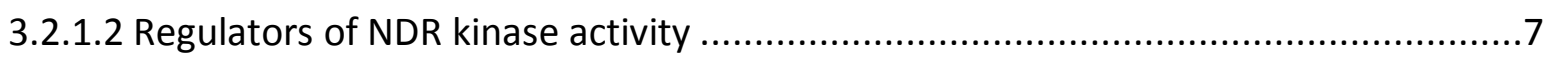

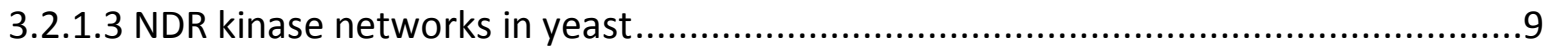

3.2.1.4 NDR kinase networks in filamentous fungi and higher eukaryotes......................11

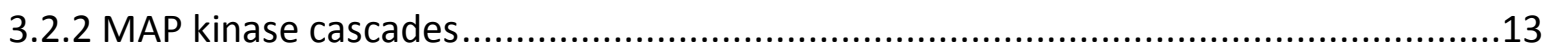

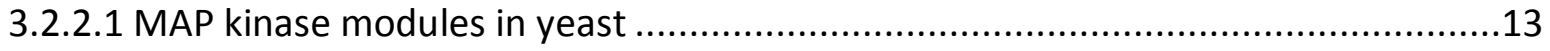

3.2.2.2 MAP kinase cascades in filamentous fungi ......................................................14

3.2.2.3 MAP kinase modules in higher eukaryotes ...................................................16

3.3 Cross-communication between NDR kinase signaling, PKA and MAP kinases .............17

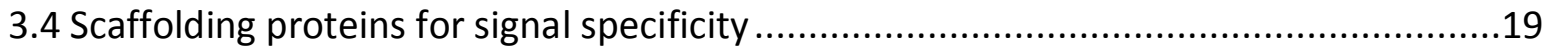

3.4.1 The scaffold protein Ste5p controls mating in budding yeast..............................19

3.4.2 Members of the Mo25 protein family acting as master regulators of Ste20 kinases .21

3.4.3 Striatin proteins acting as scaffold proteins and thereby establishing a platform for various protein protein interactions in eukaryotes ......................................................22

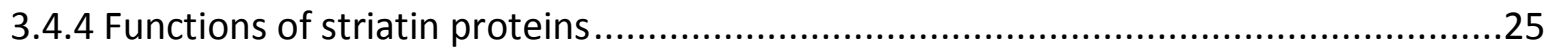

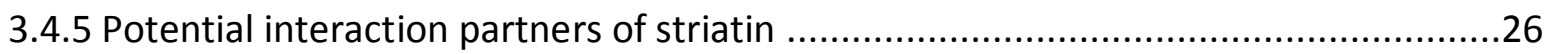

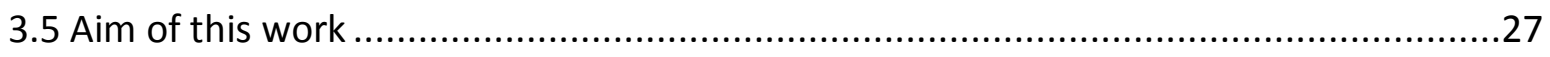

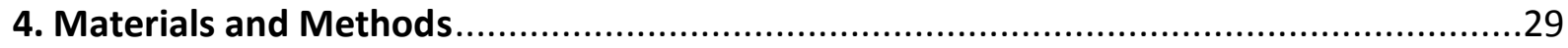

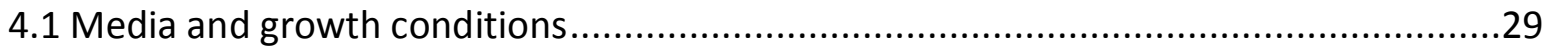

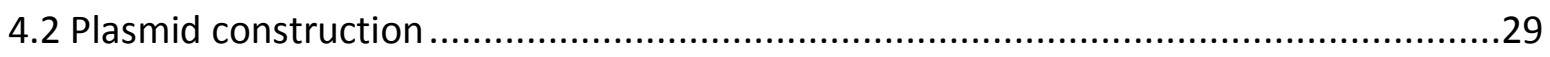

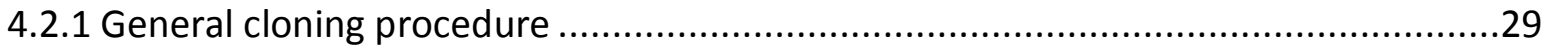

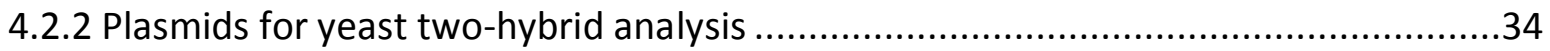

4.2.3 Constructs for overexpression of epitope-tagged fusion proteins for co-

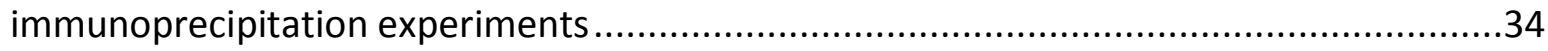

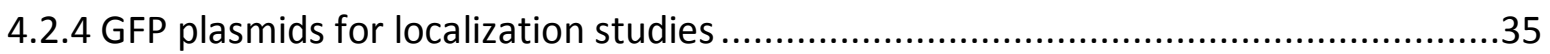


4.2.5 Dominant-active constructs of MAPKKK NRC1 and MAPKK MEK2 and a kinase-dead

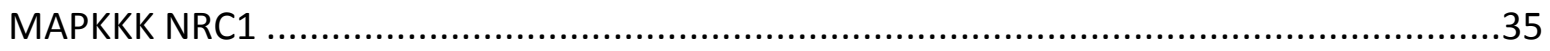

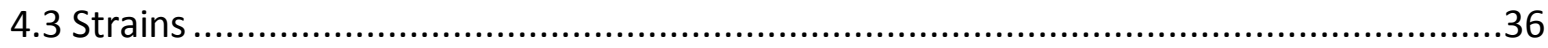

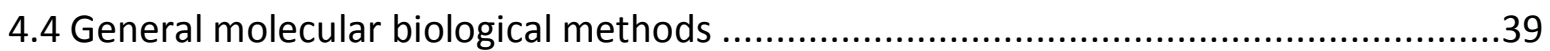

4.4.1 Genomic DNA preparation and Southern hybridization .......................................39

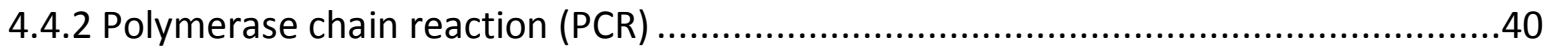

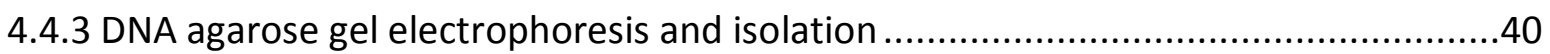

4.4.4 Enzymatic digestion of DNA and modification ...............................................41

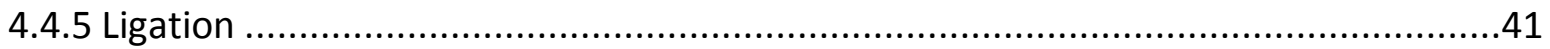

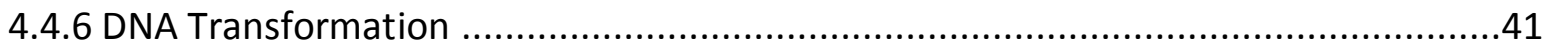

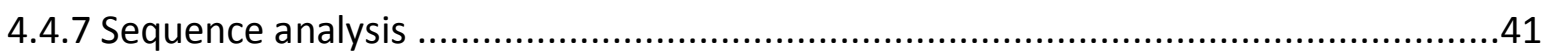

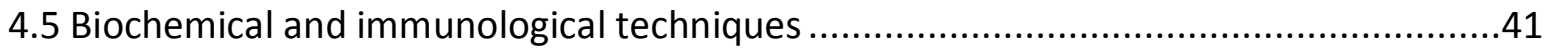

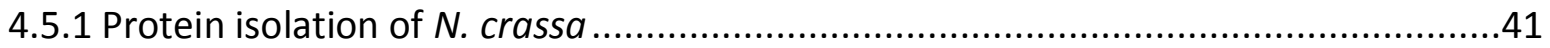

4.5.2 Separation of proteins by SDS polyacrylamide gel electrophoresis (SDS-PAGE) and

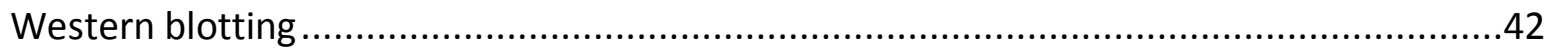

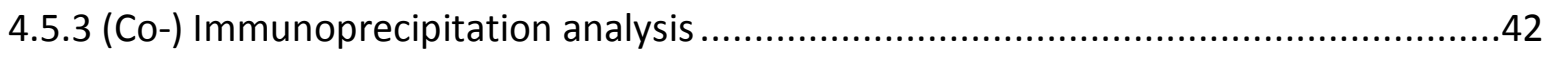

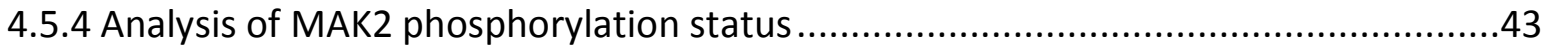

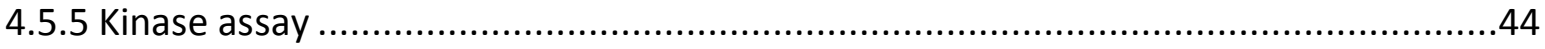

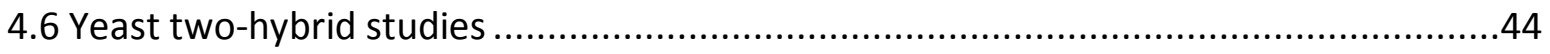

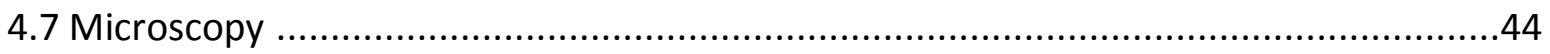

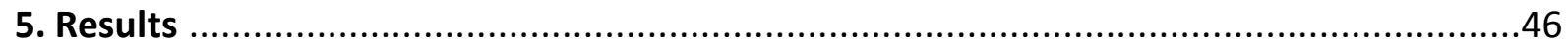

5.1 Analysis of the COT1 kinase network in the fungus $N$. crassa .................................46

5.1.1 The СОT1-МОВ2 complex is important for polar growth ..................................46

5.1.2 Activation of the NDR kinase COT1 by its upstream kinase POD6 regulates COT1

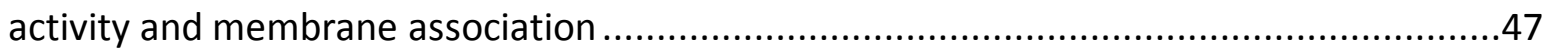

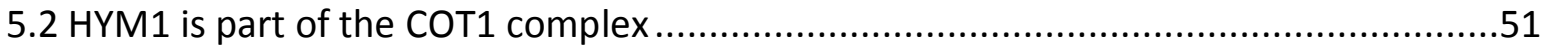

5.2.1 HYM1 acts as a scaffold protein of COT1-POD6 .................................................51

5.2.2 The HYM1-COT1 complex localizes to septa and the hyphal apex ..........................53

5.2.3 $\Delta$ hym-1 displays phenotypic defects of MAK2 pathway mutants ............................54

5.2.4 HYM1 functions as a linker protein for the MAK2 pathway ..................................58

5.2.5 Characterization of NDK1 and GCK1, potential HYM1 interaction partners..............60

5.3 Regulation of the MAK2 pathway by the Striatin complex .......................................68

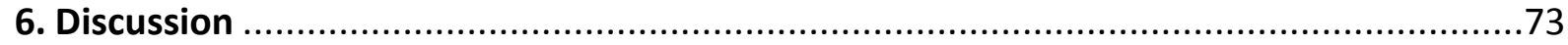

6.1 COT1 dimerization and interaction with $\mathrm{MOB}$ requires overlapping regions.................73 
6.2 Threonine phosphorylation of the NDR kinase COT1 by the upstream kinase POD6 is

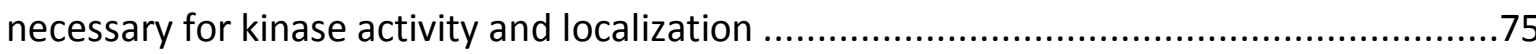

6.3 HYM1 acts as a dual scaffold for the COT1 complex and the MAK2 cascade ...............77

6.4 HYM1 functions as a master regulator of STE20 kinases .......................................79

6.5 HAM3, the $N$. crassa striatin homologue negatively regulates the MAK2 pathway ......81

6.6 Summarizing model of the cross-communication between NDR signaling, MAP kinase cascade and striatin complex that regulate sexual development and polar growth in $N$.

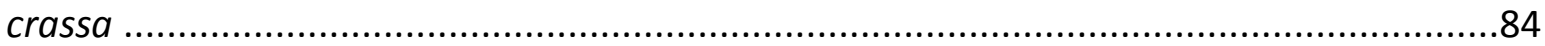

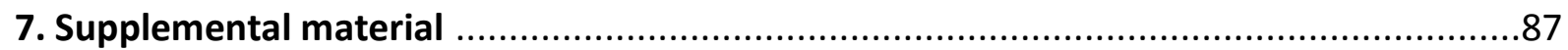

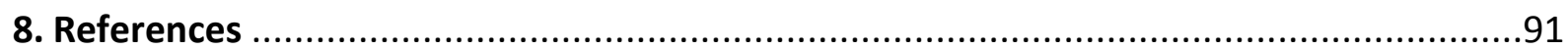

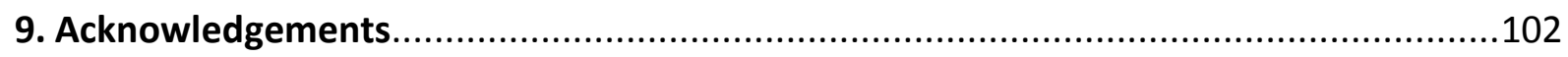

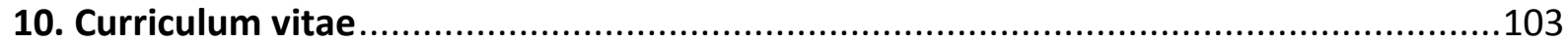




\section{List of Abbreviations}

\begin{tabular}{|c|c|}
\hline aа & amino acid \\
\hline$A D$ & activation domain \\
\hline APS & ammonium persulfate \\
\hline ATP & adenosine triphosphate \\
\hline $\mathrm{BD}$ & DNA-binding domain \\
\hline bp & base pair \\
\hline cDNA & complementary DNA \\
\hline DA & dominant-active \\
\hline DN & dominant-negative \\
\hline DNA & deoxyribonucleic acid \\
\hline dNTP & deoxyribonucleotide triphosphate \\
\hline DTT & dithiotreitol \\
\hline E. coli & Escherichia coli \\
\hline EDTA & ethylenediaminetetraacetic acid \\
\hline FGSC & Fungal Genetic Stock Center \\
\hline GCK & germinal centre kinase \\
\hline GFP & green fluorescent protein \\
\hline HA & hemagglutinin \\
\hline IP & immunoprecipitation \\
\hline kD & kiloDalton \\
\hline KD & kinase death \\
\hline LB & Luria Broth \\
\hline MAPK & mitogen-activated protein kinase \\
\hline Mst & mammalian Ste20-type \\
\hline $\mathrm{MW}$ & molecular weight \\
\hline $\mathrm{NCBI}$ & National Center for Biotechnology Information \\
\hline N. crassa & Neurospora crassa \\
\hline NDPK/NDK & nucleoside-diphosphate kinase \\
\hline NDR & nuclear Dbf2-related \\
\hline NLS & nuclear localization signal \\
\hline NP-40 & Nonidet P-40 \\
\hline OD & optical density \\
\hline PAGE & polyacrylamide gel electrophoresis \\
\hline PAK & p21-activated kinase \\
\hline PBS & phosphate buffered saline \\
\hline PCR & polymerase chain reaction \\
\hline PKA & protein kinase $A$ \\
\hline $\mathrm{pm}$ & palmitylation \\
\hline MOB & Mpsl one binder \\
\hline RAM & regulation of morphogenesis and Ace $2 p$ \\
\hline RFP & red fluorescent protein \\
\hline RNA & ribonucleic acid \\
\hline S. cerevisiae & Saccharomyces cerevisiae \\
\hline SD & Synthetic Defined \\
\hline SDS & sodium dodecyl sulfate \\
\hline
\end{tabular}


Ser

S. macrospora

S. pombe

TEMED

Thr

UTR

UV

VMM

WB

YEPD serine

Sordaria macrospora

Schizosaccharomyces pombe

tetramethylethylenediamine

threonine

untranslated region

ultraviolet

Vogel's Minimal Medium

Western blot

Yeast Extract Peptone Dextrose 


\section{Summary}

Establishment and maintenance of cellular polarity are important and fundamental processes in eukaryotes, which have to be coordinated with cell division, differentiation, and cell growth. Various signal transduction pathways like PKA (protein kinase A) signaling, NDR (nuclear Dbf2-related kinases) pathway or MAPK (mitogen-activated protein kinase) cascades regulate polarized growth to allow proper development of cells and organisms. In the filamentous fungus Neurospora crassa the NDR kinase COT1 is involved in the coordination of polar hyphal tip extension and loss of this kinase leads to cessation of hyphal tip extension and formation of a hyperbranched mycelium. NDR kinases gain their full kinase activity by phosphorylation at two conserved sites, the activation loop in the $\mathrm{N}$-terminus and the hydrophobic motif in the C-terminus. During this work I was able to show that COT1 forms homodimers via a conserved $\mathrm{N}$-terminal extension, which is also required for the interaction of the kinase with its adaptor proteins MOB2A and MOB2B. These exclusive interactions allow the formation of distinct homo- and heterocomplexes with different COT1 kinase activities. The phosphorylation of Thr589 in the hydrophobic motif of COT1 by the upstream kinase POD6 further stimulates COT1 by induction of a conformational change in the kinase. Further, Thr589 phosphorylation is not only important for maximal in vitro kinase activity, but also a critical signal for targeting the active COT1-MOB complex to the site of cell growth at the hyphal apex.

The MO25 homologue HYM1 acts as a potential scaffold protein between COT1 and POD6 verified by yeast two-hybrid and co-immunoprecipitation approaches. Moreover, GFP-fusion proteins of HYM1 and COT1 showed the same localization at septa and the hyphal apex. However, the $\Delta h y m-1$ phenotype is distinct from the typical "barbed wired" defect displayed by the central COT1 complex mutants but phenocopies the defects of mutants of the MAK2 kinase pathway. I could show interactions between HYM1 and proteins of the MAK2 cascade and further that the MAK2 activity is abolished in a $\triangle h y m-1$ background suggesting that HYM1 acts not only as the potential scaffold protein between COT1 and POD6 but is also important for the signal transduction between the MAP kinases in the MAK2 kinase cascade. By a HYM1 yeast two-hybrid screen two additional proteins, GCK1 and NDK1, could be determined as HYM1 interacting proteins which seem to act as modulators in the COT1MAK2 pathway.

Phenotypical characterization of the $\Delta h y m-1, \Delta m a k-2, \Delta n d k-1$ and $\Delta g c k-1$ deletion strains exhibited overlapping developmental defects suggesting a cross-communication between these proteins. Additionally, literature studies described a link between NDK1, GCK1, MAP kinases and the striatin complex which play important roles in endocytosis, signal transduction and cell adhesion. The striatin homologue HAM3 in N. crassa and its interaction partner МOB3 display the same developmental and morphological defects like observed for $\triangle$ hym-1 and MAP kinase mutants. In addition, the MAK2 activity is increased in ham-3 and mob-3 mutants indicating a negative regulation of the MAK2 cascade. Studies of GFP-tagged GCK1, NDK1, HAM3 and MOB3 constructs reveal partial overlapping, but also different localizations excepting $\mathrm{HAM} 3$ and MOB3 which display exactly the same localization at nuclear envelopes suggesting that $\mathrm{HAM} 3$ and $\mathrm{MOB} 3$ acting in the same pathway.

To sum up, interactions between the COT1 signaling, the MAK2 kinase pathway and the striatin complex could be determined suggesting the occurrence of different partial overlapping complexes which are important for sexual development and polar growth. 


\section{Zusammenfassung}

In Eukaryoten ist die Etablierung und Aufrechterhaltung von Zellpolarität ein wichtiger und grundlegender Prozess, welcher mit Zellteilung, Differenzierung und Zellwachstum koordiniert werden muss. Vielfältige Signaltransduktionswege wie z.B. der PKA Signalweg (Proteinkinase A), der NDR (nukleäre Dbf2-verwandte Kinase) Kinaseweg oder die MAPK Kaskade (mitogen aktivierte Proteinkinase) regulieren polares Wachstum um die korrekte Entwicklung von verschiedenen Zellen und Organismen zu ermöglichen.

In dem filamentösen Pilz Neurospora crassa ist die NDR Kinase COT1 an der Koordination des polaren Hyphenwachstums beteiligt und der Verlust dieser Kinase führt zur Einstellung des polaren Hyphenspitzenwachstums und der Ausbildung eines hyperverzweigten Mycels.

NDR Kinasen erlangen ihre vollständige Kinaseaktivität durch Phosphorylierung an zwei konservierten Resten; im Aktivierungssegment des N-Terminus und in dem hydrophoben Motiv des C-Terminus. Während dieser Arbeit konnte ich zeigen, dass COT1 Homodimere über die konservierte $\mathrm{N}$-terminale Verlängerung der regulatorischen Domäne bildet, die außerdem nötig für die Interaktion zwischen der Kinase und dessen Adaptorproteine MOB2A und MOB2B ist. Diese Interaktionen ermöglichen die Bildung verschiedener Homo- und Heterokomplexe mit verschiedenen Kinaseaktivitäten. Die Phosphorylierung von COT1 am Thr589 im hydrophoben Motiv durch die übergeordnete Kinase POD6, bewirkt eine Konformationsänderung und stimuliert die COT1 Kinaseaktivität. Desweiteren ist die Thr589 Phosphorylierung nicht nur für die maximale in vitro Kinaseaktivität wichtig, sondern auch für den Transport des aktiven СОT1-MOB Komplexes an die Hyphenspitze.

Das MO25 Homolog HYM1 fungiert als mögliches Gerüstprotein zwischen COT1 und POD6, was durch Hefe-Zwei-Hybrid- und Co-Aufreinigungsversuche verifiziert wurde.

Außerdem zeigen COT1 und HYM1 GFP-Fusionsproteine die gleiche Lokalisation an Septen und an der Hyphenspitze. Dennoch unterscheidet sich der $\Delta h y m-1$ Phänotyp von dem typischen "stachelartigen" Defekt der Mutanten des COT1 Komplexes, zeigt jedoch typische Deletionsdefekte der MAK2-Komplex Mutanten. Darüber hinaus konnte ich Interaktionen zwischen HYM1 und den Proteinen der MAK2 Kaskade zeigen und desweiteren, dass die MAK2 Aktivität in $\triangle h y m-1$ nicht mehr vorhanden ist. Dies führt zu der Annahme, dass HYM1 nicht nur als mögliches Gerüstprotein zwischen COT1 und POD6 fungiert, sondern auch für die Signalweiterleitung innerhalb der MAP Kinasekaskade wichtig ist.

Durch ein HYM1 Hefe-Zwei-Hybrid Experiment konnte ich zwei zusätzliche Proteine, GCK1 und NDK1, als HYM1 interagierende Proteine identifizieren, die anscheinend als Modulatoren des COT1-MAK2 Signalweges agieren. Eine phänotypische Charakterisierung von $\Delta h y m-1, \Delta m a k-2, \Delta n d k-1$ and $\Delta g c k-1$ zeigt überlappende Entwicklungsdefekte, die eine Verbindung zwischen diesen Proteinen annehmen lassen. Außerdem beschreiben Literaturstudien einen Zusammenhang zwischen NDK1, GCK1, der MAP Kinasekaskade sowie des Striatinkomplexes, welcher wichtige Rollen in der Endozytose, Signalweiterleitung und Zelladhäsion übernimmt. HAM3, das Striatinhomolog in $N$. crassa und dessen Interaktionspartner MOB3 zeigen dieselben Entwicklungs- und Morphologiedefekte, die man auch bei den $\Delta h y m-1$ und mak-2 Mutanten beobachten kann. Zusätzlich ist die Mak2 Aktivität in den Deletionsmutanten ham-3 und mob-3 erhöht, was zur Annahme führt, dass es sich hierbei um eine negative Regulation der MAK2 Kaskade handelt.

Lokalisationsstudien der GFP-Epitop markierten Konstrukte von GCK1, NDK1, HAM3 und MOB3 zeigen teilweise überlappende aber auch unterschiedliche Lokalisationsmuster, ausgenommen HAM3 und MOB3. Diese zeigen eine identische Lokalisation an 
Zellkernhüllen, was zur Annahme führt, dass HAM3 und MOB3 in demselben Signalweg agieren.

Zusammengefasst konnten Interaktionen zwischen dem COT1 Signalweg, der MAK2 Kinasekaskade und dem Striatinkomplex beschrieben werden. Diese Interaktionen weisen auf das Vorhandensein von partiell überlappenden Komplexen hin und sind wahrscheinlich essentiell für die sexuelle Entwicklung und das polare Wachstum in dem filamentösen Pilz Neurospora crassa. 


\section{Introduction}

\subsection{Polar growth in filamentous fungi}

The simple mode of growth of filamentous fungi has made them an outstanding system for the analysis of cellular processes. Due to the fast growth rate ( $\geq 1 \mu \mathrm{m} / \mathrm{sec}$ (Seiler \& Plamann, 2003)) and the highly elongated form, filamentous fungi represent a prime example to analyze polarized growth. This process is based on an interplay between the cytoskeleton, marker proteins and membrane domains. A continuous flow of secretory vesicles from the hyphal cell body to the growing tip is essential for cell wall and membrane extension (Fischer et al., 2008). Microtubules (MT) and actin, together with their corresponding motor proteins, are involved in this process and the arrangement of the cytoskeleton is crucial to establish and maintain polarity. The rapid rate of hyphal growth is driven by the Spitzenkörper which is closely connected with cell morphogenesis and polar growth, and is only present at actively growing sites (Harris, 2006, Harris \& Momany, 2004, Sudbery \& Gladfelter, 2008). The core components involved in generating cell polarity are conserved from yeast to mammals (Harris, 2006, Harris \& Momany, 2004).

A first model to understand the mechanism of polarized growth has been exhibited by studies in the ascomycete Saccharomyces cerevisiae. After a period of isotropic expansion unbudded yeast cells switch to polar growth which leads to bud emergence. After that cycle yeast again reverts to isotropic growth and the bud expands until cell separation occurs (Momany, 2002).

In filamentous fungi the process of polar growth starts with isotropic expansion of a spore. Then the side of germ tube emergence is selected, thereby delivering materials and proteins needed for establishing the new plasma membrane and cell wall. Finally, the germ tube emerges and extends apically. In contrast to yeast, hyphal compartments partitioned by septa, remain attached and a new site for branch emergence is selected. Moreover, the polar growth continues along the main hypha and begins to branch (Momany, 2002) (Figure 1).

Understanding the establishment and maintenance of polarity, hyphal elongation, septation, branching and differentiation are at the core of current researches (Riquelme et al., 2011). 


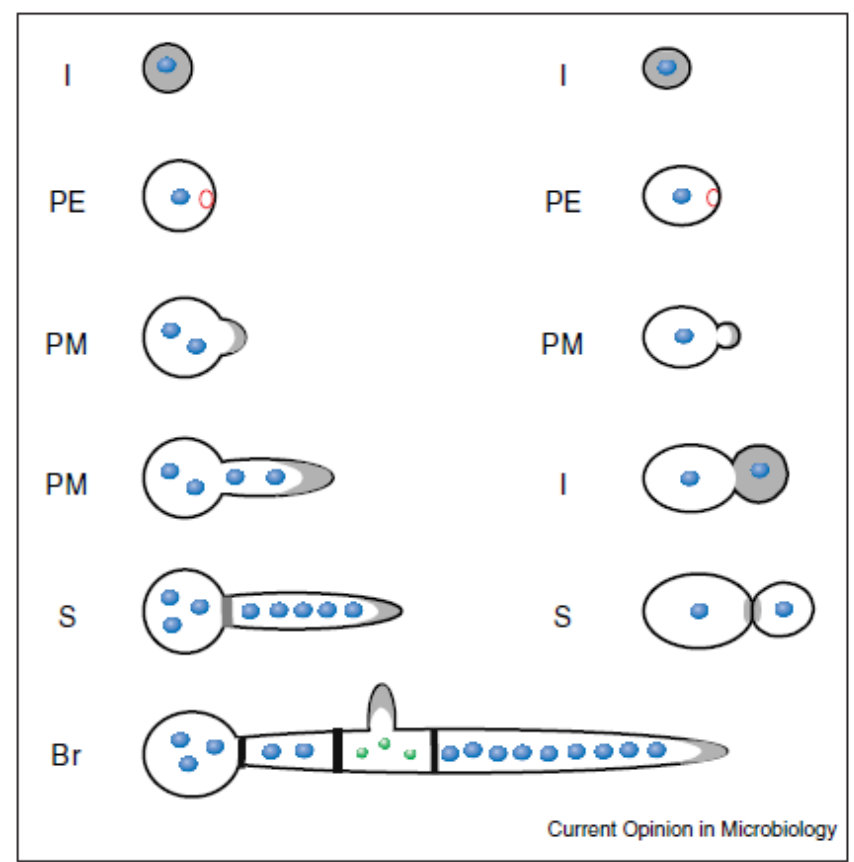

Figure 1: Comparison of morphogenesis between filamentous fungi and budding yeast. Gray shading shows areas of growth. I, isotropic expansion; PE, polarity establishment; PM, polarity maintenance; S, septation; $\mathrm{Br}$, branching. Blue ovals represent interphase nuclei; green dots, mitotic nuclei and the small open red circles, cortical markers (Momany, 2002).

\subsection{General signaling cascades that regulate polar growth (NDR, MAPK)}

The hallmark of filamentous fungi is the apical tip extension, leading to the formation of elongated hyphae. Along with neurons and pollen tubes, hyphae represent the most highly polarized cells (Borkovich et al., 2004, Harris, 2006, Palanivelu \& Preuss, 2000). Polarized growth is a complex multifactorial property, which is coordinated by numerous conserved signaling pathways, such as the mitogen-activated protein kinase (MAPK), cAMP-dependent protein kinase (PKA) or the nuclear Dbf2-related (NDR) kinase pathways (Hergovich et al., 2006b, Lengeler et al., 2000, Lewis et al., 1998). These pathways are also important for maintaining pathogenicity and development (D'Souza et al., 2001, Monge et al., 2006, Xu, 2000, Xu et al., 2007).

\subsubsection{NDR kinases}

The nuclear Dbf2-related (NDR) kinases play an important role in regulation of cellular polarity and cell division. These kinases belong to the AGC class (CAMP-dependent protein kinase $A$, cGMP-dependent protein kinase $G$ and phospholipid-dependent protein kinase $C$ ) 
of serine/threonine protein kinases based on their structural similarities within their kinase domains and are highly conserved from yeast to human (Hanks \& Hunter, 1995, Manning et al., 2002, Millward et al., 1995). Within their catalytic domain an insert of 30-60 amino acids exists (Bidlingmaier et al., 2001, Millward et al., 1995, Verde et al., 1998, Yarden et al., 1992) which thought to possess an auto-inhibitory function that is mediated through the high content of basic (positively charged) amino acids in its C-terminal region (Bichsel et al., 2004). The human NDR kinase NDR1 harbors a conserved basic region at the N-terminus of the kinase domain which was found to function as a dimerization domain and binding platform for other regulatory proteins like the MOBs (Mps1 one binder) (He et al., 2005, Hergovich et al., 2006a, Hergovich et al., 2006b, Hou et al., 2004, Millward et al., 1998, Ponchon et al., 2004) (Figure 2). NDR kinase complexes occur in the RAM (regulation of

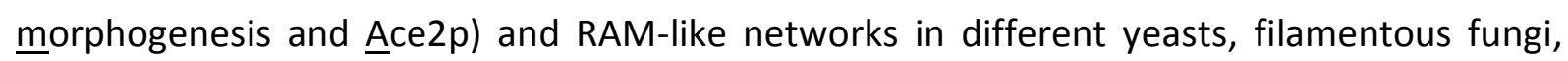
Drosophila's Hippo-pathway and the NDR pathway in mammals.

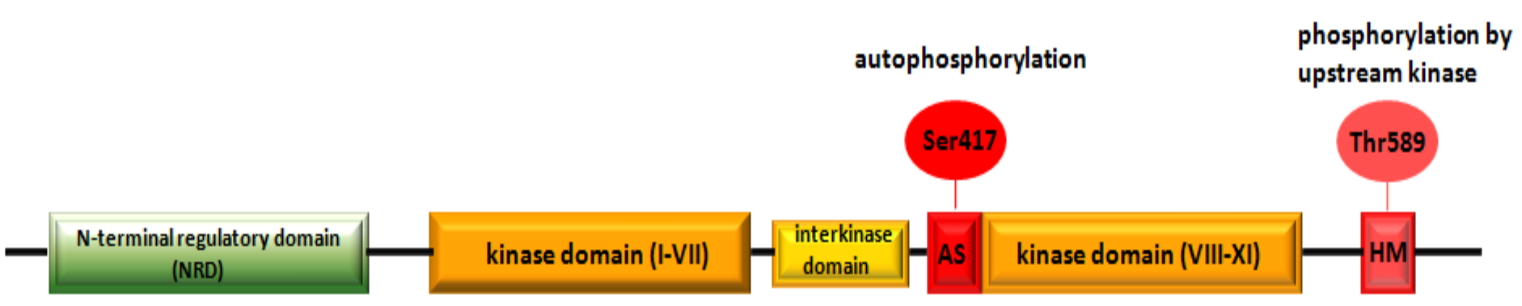

Figure 2: General domain structure of NDR kinases. At the N-terminus of the kinase domain a conserved basic region functions as dimerization domain and binding platform for other regulatory proteins (e.g. MOBs). Between the kinase domains VII and VIII an insert of 30-60 amino acids exists (interkinase domain). The activation segment (AS) containing a conserved serine residue is important for autophosphorylation. The NDR kinase becomes fully active by an additional phosphorylation of the threonine residue in the hydrophobic motif (HM) at the C-terminus through an upstream kinase.

\subsubsection{Activation of NDR kinases}

NDR kinases gain their full kinase activity by phosphorylation at two conserved sites, the activation loop and the hydrophobic motif. The activation segment in the $\mathrm{N}$-terminal lobe of the kinase domain carries a conserved serine or threonine residue which needs to be autophosphorylated to mediate full catalytic activity. In addition to the phosphorylation event in the activation segment, most NDR kinases require a second phosphorylation step at the C-terminal hydrophobic motif by an upstream kinase to gain full catalytic activity (Keranen et al., 1995, Stegert et al., 2005, Yang et al., 2002). Structural analyses demonstrated that the phosphorylated hydrophobic motif folds back and interacts with the 
hydrophobic pocket in the N-terminal lobe (Biondi \& Nebreda, 2003, Frodin et al., 2002, Kannan et al., 2007, Yang et al., 2002). Conclusively, the phosphorylated hydrophobic motif together with the phosphorylation of the activation loop results in conformational modifications of the $\mathrm{N}$ - and C-terminal lobes of the kinase. Subsequently, stabilization of the active conformation leads to a fully active kinase. For different NDR kinases like baker's yeast Cbk1p and human NDR1/2 it has been shown that the serine residue in the activation segment is modulated by autophosphorylation (Jansen et al., 2006, Stegert et al., 2004, Tamaskovic et al., 2003) and the threonine residue in the hydrophobic motif of several NDRs is targeted by Ste20-like kinases which belong to the subgroup of GCKs (Chan et al., 2005, Emoto et al., 2006, Stegert et al., 2005).

\subsubsection{Regulators of NDR kinase activity}

The function and localization of the NDR kinase COT1 in N. crassa is controlled by its binding partner MOB2A and МOB2B (Maerz et al., 2009) and through an upstream mammalian Ste20-type (Mst) kinase of the Ste20 superfamily POD6 (Seiler et al., 2006).

The MOB (ㅁps1 one binder) proteins are characterized by their mob/phocein domain and are highly conserved in all eukaryotes. The protein family can be divided into three subgroups due to sequence comparisons of the MOB core domain - the MOB1-like and the MOB2-like (according to their relationship with Saccharomyces cerevisiae Mob1p or Mob2p), and the MOB3/phocein like proteins (Mrkobrada et al., 2006). The MOB3/phocein subfamily as the most divergent group was described as a component of several complexes consisting of striatin, protein phosphatase $\underline{2 A}$ (PP2A) and germinal çentre kinases (GCK), but they also interact with a nucleoside-diphosphate kinase and dynamin (Baillat et al., 2002, Baillat et al., 2001, Benoist et al., 2006, Goudreault et al., 2009). The MOB1- and MOB2-like proteins bind to NDR kinases and are essential for stimulating the catalytic kinase activity (Bichsel et al., 2004, Hou et al., 2004, Weiss et al., 2002).

In addition to the MOB proteins, a second group of NDR kinase interacting proteins, the GCK family, is important for NDR kinase activity and function (Chan et al., 2005, Emoto et al., 2006, Nelson et al., 2003, Stegert et al., 2005, Walton et al., 2006). GCKs together with the PAKs (p21-activated kinases) belong to the group of Ste20-related kinases, which are involved in different cellular and developmental processes such as morphogenesis, cell cycle 
regulation, apoptosis and acting as potential regulators of the MAP kinase cascades. Both kinase subfamilies can be distinguished by the position of their kinase domains: the PAKs possess a C-terminal kinase domain, while the catalytic domain of GCKs resides at the $\mathrm{N}$ terminus (Dan et al., 2001) (Figure 3).

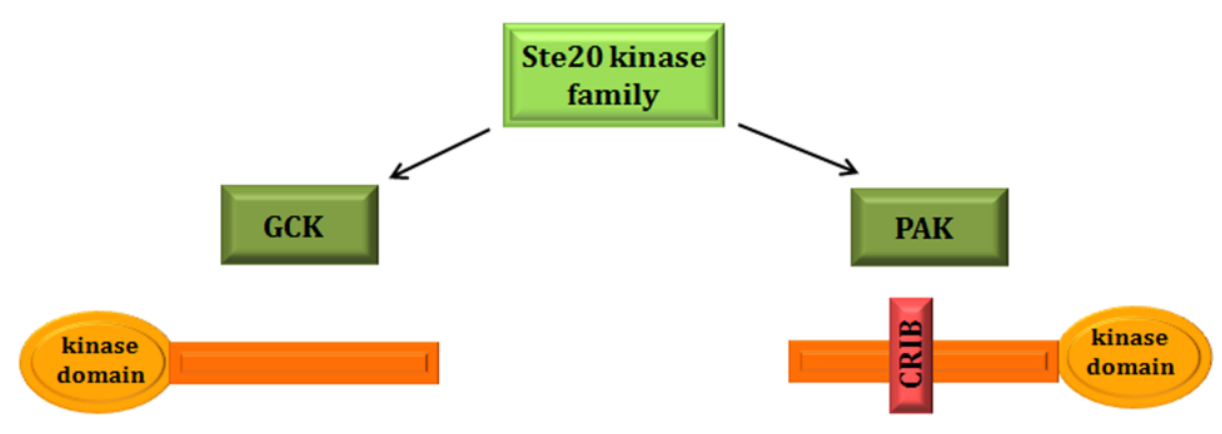

Figure 3: The Ste20 kinase family can be subdivided into GCKs and PAKs based on position of the kinase domain and the presence of the CRIB domain, which is restricted to the PAK kinases.

Moreover, the PAK family members contain a CRIB (Cdc42/ Rac binding) domain in the Nterminal non-catalytic domain which is important for binding to small GTPases. In contrast, the GCK family is highly diverse and can be subdivided into eight groups due to their domain structure within the regulatory C-terminus (Cybulsky et al., 2009). Only members of the subgroups II and III, e.g. Drosophila's Hippo (Hpo), mammalian Mst1, Mst2, Mst3 or fungal Kic1p (Sc), Nak1 (Sp) and POD6 (Nc) are described as interactors of NDR kinases and are involved in the phosphoregulation of these kinases (Table 1). 
Table 1: Overview of NDR kinases, the corresponding upstream kinases and their functions in different organisms.

\begin{tabular}{|c|c|c|c|}
\hline NDR kinase & upstream kinase & function & organism \\
\hline Cbk1p & Kic1p & $\begin{array}{c}\text { part of the RAM } \\
\text { network }\end{array}$ & S. cerevisiae \\
\hline Orb6 & Nak1/ Orb3 & $\begin{array}{c}\text { part of the MOR } \\
\text { network }\end{array}$ & S. pombe \\
\hline COT1 & POD6 & polar growth & N. crassa \\
\hline Trc & Hро & $\begin{array}{c}\text { dendritic tiling, } \\
\text { epidermal and } \\
\text { neurite outgrowth, } \\
\text { cell shape }\end{array}$ & D. melanogaster \\
\hline NDR1/2 & MST1/2/3 & $\begin{array}{l}\text { neurite outgrowth, } \\
\text { centrosome } \\
\text { duplication }\end{array}$ & H. sapiens \\
\hline
\end{tabular}

\subsubsection{NDR kinase networks in yeast}

NDR kinases are important for correct cell differentiation and polar morphogenesis in various organisms, but their specific functions are still elusive (Geng et al., 2000, Racki et al., 2000, Yarden et al., 1992, Zallen et al., 2000) summarized in Hergovich et al., 2006b.

The yeasts $S$. cerevisiae and S. pombe express two distinct NDR kinase networks, consisting of distinct NDR kinases, MOB proteins and upstream kinases.

One signaling cascade is involved in coupling the cell cycle with cell separation and termed mitotic exit network (MEN) in budding and septation initiation network (SIN) in fission yeast (Bardin \& Amon, 2001, de Bettignies \& Johnston, 2003, Krapp et al., 2004, Krapp \& Simanis, 2005, Krapp \& Simanis, 2008, Roberts-Galbraith \& Gould, 2008).

The second NDR kinase network is involved in the regulation of morphology and polar growth and is called RAM (regulation of morphogenesis and Ace2p) in baker's yeast and MOR ( morphogenesis of $\underline{\text { Orb6) }}$ in fission yeast and filamentous fungi (Bogomolnaya et al., 2006, Maerz \& Seiler, 2010, Nelson et al., 2003, Verde et al., 1998).

The RAM pathway in the budding yeast consists of the NDR kinase Cbk1p, the MOB protein Mob2p and Kic1p, the GC upstream kinase of Cbk1p. By yeast two-hybrid studies and copurification experiments additional network components such as Tao3p and Hym1p which are two potential scaffolding proteins, Sog2p, a protein of unknown function, and the transcription factor Ace2p, a downstream effector of Cbk1p, were identified (Ho et al., 2002, 
Ito et al., 2001, Kurischko et al., 2005, Nelson et al., 2003). Furthermore, interaction analysis revealed an interaction between Kic1p and Hym1p rather Sog $2 p$ and the scaffold Tao3p forms a complex together with Kic1p and Cbk1p linking the two kinases. Moreover, Cbk1p bound to Mob2p is able to associate with and phosphorylate the transcription factor Ace $2 p$. Dysfunction of one of these morphogenesis network components leads to a loss of cellular polarity accompanied by a cell separation defect visible by the round yeast cell morphology and clustered growth (Colman-Lerner et al., 2001, Nelson et al., 2003, Racki et al., 2000, Weiss et al., 2002).

In S. pombe the MOR network consists of the NDR kinase Orb6 and the kinase-associated protein Mob2 (Hou et al., 2003). The Kic1-homologue Nak1 acts upstream of Orb6 and the Orb6-Nak1 complex binds to the Tao3p homologue Mor2 (Kanai et al., 2005). A Hym1p homologue and Nak1 interacting and activating protein Pmo25 is also present, whereas a Sog2p-homologue could not be found in the MOR network of fission yeast. Deletions or conditional mutants of these components are characterized by apolarly growing cells or spherically germinating spores, which lyse after a few rounds of cell division (Hirata et al., 2002, Hou et al., 2003, Kanai et al., 2005, Verde et al., 1998) (Figure 4).

In conclusion, most components of the RAM/MOR networks are conserved with some exceptions (e.g. Sog2p) in both yeasts. 

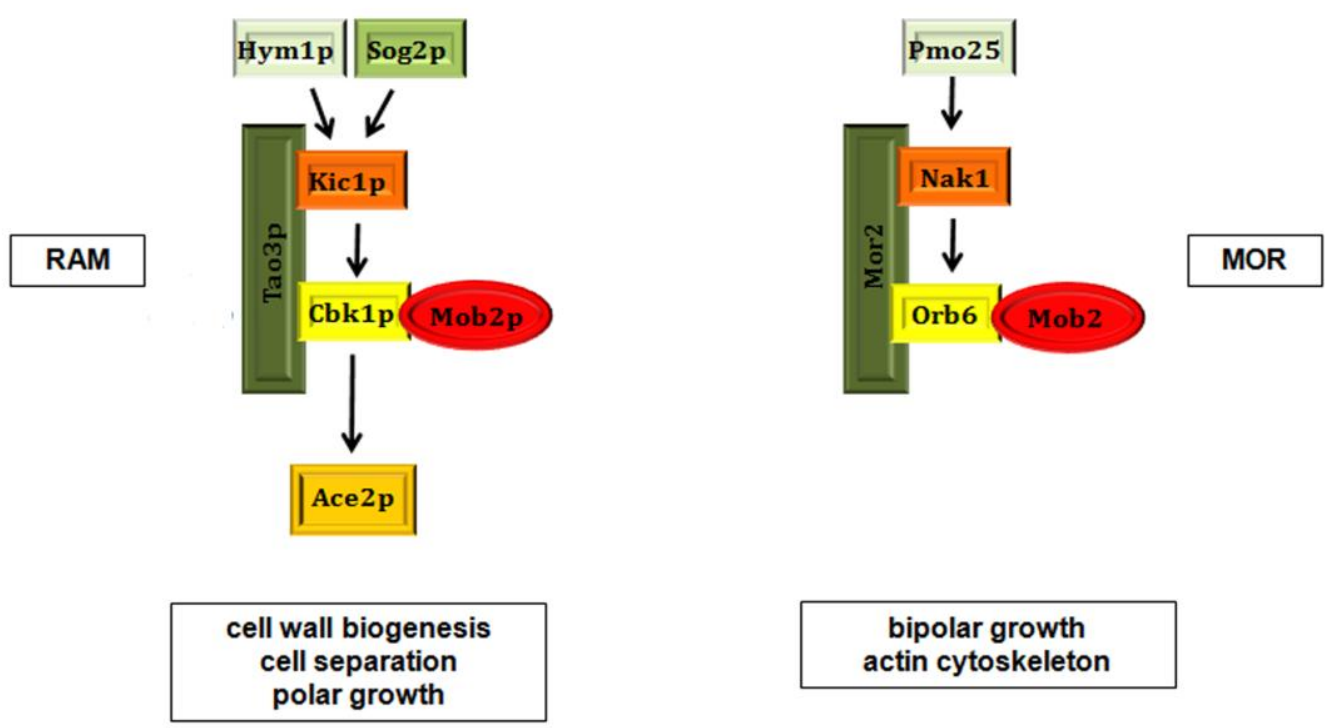

Figure 4: Schematic model of the RAM network in S. cerevisiae and the MOR network in S.pombe.

\subsubsection{NDR kinase networks in filamentous fungi and higher eukaryotes}

Components of the RAM/MOR networks are also highly conserved and are described in other fungi, such as Aspergillus nidulans, Neurospora crassa, Cryptococcus neoformans or the fungal pathogens Candida albicans, Ustilago maydis and Claviceps purpurea (McNemar \& Fonzi, 2002, Song et al., 2008, Walton et al., 2006) (Table 2). The morphological output can be different while the network components are conserved. In Cryptococcus neoformans for example, the loss of RAM components do not result in loss of polarity as observed for baker's and fission yeast, whereas in $N$. crassa and $A$. nidulans for example the respective NDR kinases COT1 and COTA are both involved in the maintenance of polar growth (Johns et al., 2006, Yarden et al., 1992).

Table 2: Overview about the conserved RAM/MOR network components in the filamentous fungi $A$. nidulans and $\mathbf{N}$. crassa in comparison to $S$. cerevisiae and S. pombe

\begin{tabular}{|ccccc}
\hline & S. cerevisiae & S. pombe & A. nidulans & N.crassa \\
\hline & Hym1p & Pmo25 & HymA & HYM1 \\
Tao3p & Mor2 & ANID_00594.1 & TAO3 \\
\hline RAM/MOR & Mob2p & Mob2 & ANID_01370.1 & MOB2A \\
\hline & Cbk1p & Orb6 & MOB2B \\
& Kic1p & Nak1 & ANID_05674.1 & COT1 \\
& & &
\end{tabular}


Loss of function of these proteins results in compact colony growth and hyperbranched hyphae due to cessation of tip extension and excessive induction of hyphal tip formation (Shi et al., 2008). N. crassa COT1 was described to associate with the potential upstream kinase POD6 (Seiler et al., 2006) and temperature sensitive mutants of both exhibit the same hyperbranched and compact phenotype at restrictive temperature (Figure 5). According to this, mutation or deletion of cpcot-1, a NDR gene of the pathogen fungus $C$. purpurea results also in a hyperbranched, and compact growing phenotype (Scheffer et al., 2005). In the dimorphic fungi $C$. albicans and U. maydis disruption of the NDR kinase genes CBK1 and $u k c 1$ cause cell separation defects and a block in the transition from budding to hyphal growth (Durrenberger \& Kronstad, 1999, McNemar \& Fonzi, 2002, Song et al., 2008). To sum up, it has been shown that Cot-1 homologues in other filamentous fungi are involved in hyphal elongation and in restricting excessive branch formation, supporting the significance of this kinase in growth of filamentous fungi.
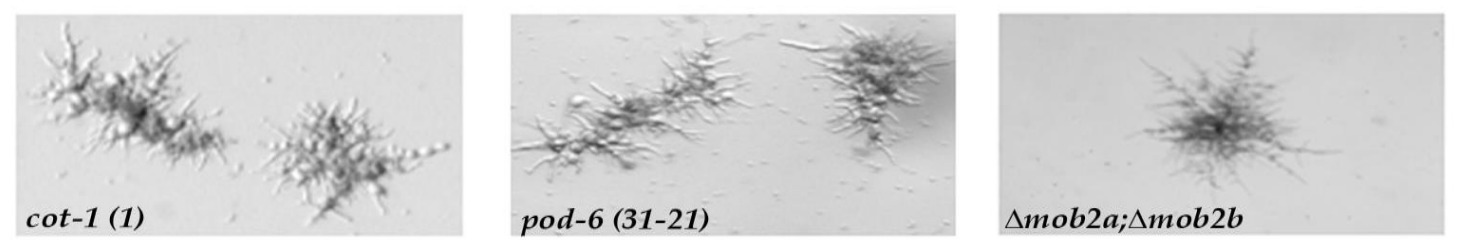

Figure 5: Temperature-sensitive mutant strains of cot-1 and pod-6 (germinating at $37^{\circ} \mathrm{C}$ ) and double deletion strain $\Delta m o b 2 a ; \Delta m o b 2 b$ show defects in polar growth and hyphal tip elongation.

Some of the core components described for SIN/MEN or MOR/RAM function were also found in animals. One NDR pathway is involved in the regulation of cell shape, while the other contributes primarily to cell proliferation. However, in contrast to fungi, their functions partially overlap, and individual components are exchangeable between both pathways. The two NDR kinase modules in Drosophila melanogaster are distinguishable by their NDR kinase and the respective scaffolding protein, but each NDR kinase is activated by the same upstream germinal center kinase and can interact with the same set of MOB proteins. Additionally, the number of individual MOB proteins and NDR kinases is increased. Drosophila melanogaster exhibits at least three different MOB1/2-like genes and two NDR kinases, while mammals have more than five MOB1/2-like proteins and four NDR kinases. Thus, the NDR kinase networks in animals are more complex and highly flexible. 
In conclusion, two different NDR kinase complexes phosphorylated and activated by distinct upstream kinases and adaptor proteins are described in yeast. Moreover, most of these components are conserved in the filamentous fungi $N$. crassa and A. nidulans. Also in $D$. melanogaster two NDR signaling cascades are characterized, but in contrast to yeast the kinases are activated in the same manner. Furthermore, both kinases act in parallel to regulate tissue innervations and dendritic morphogenesis. According to this, the mammalian NDR kinase networks are organized in a similar manner compared to those of the fruit fly, but the number of NDR kinases and adaptor proteins is increased.

\subsubsection{MAP kinase cascades}

MAP kinases are arranged within protein kinase cascades and each cascade consists of no fewer than three enzymes that are activated in series by phosphorylation (Cobb, 1999, Qi \& Elion, 2005). Frequently, a fourth kinase of the Ste20/PAK group, also called MAPKKKK, is acting upstream of the MAPK signaling pathways (Dan et al., 2001). Various reports have shown that distinct MAPK pathways are regulated by cross-communication with each other and other signaling pathways summarized in Lengeler et al., 2000 and Stork \& Schmitt, 2002. For instance, yeast filamentous growth is regulated, in part, by two conserved signal transduction cascades: a mitogen-activated protein kinase cascade and a G-protein regulated cyclic AMP signaling pathway. Related signaling cascades play analogous roles in regulating mating and virulence in the plant fungal pathogen Ustilago maydis and the human fungal pathogens Cryptococcus neoformans and Candida albicans (Lengeler et al., 2000).

\subsubsection{MAP kinase modules in yeast}

The functional modules of each MAPK pathway as well as the interplay between the different signaling routes are best characterized in the unicellular ascomycete Saccharomyces cerevisiae (Bahn et al., 2007, Lengeler et al., 2000, Madhani, 2000, Pan et al., 2000). In budding yeast, the MAP kinases constitute five partially overlapping pathways regulating mating, filamentation, cell integrity, response to high osmolarity and ascospore formation. Fus $3 p$ and Kss1p regulate mating in response to peptide mating pheromones and have shared and unique substrates. Thereby only Fus3p is essential for mating and Kss1p functions in additional pathways that regulate invasive growth/pseudohyphal development 
and cell wall integrity (Bardwell, 2005, Elion et al., 2005, Wang \& Dohlman, 2004). Hog1p regulates intracellular osmolarity in response to extracellular osmolarity and citric acid stress (Brewster et al., 1993, Maeda et al., 1994, O'Rourke \& Herskowitz, 1998, Westfall et al., 2004). Mpk1p regulates cell wall integrity and budding in response to mechanical changes at the cell wall/plasma membrane (Cid et al., 1995, Kim et al., 2010, Lin \& Levin, 1990, Martin \& Wood, 2002). The Smk1p MAPK, part of the spore wall assembly pathway, is not present in growing cells but appears during sporulation and regulates that developmental process (Gustin et al., 1998, Huang et al., 2005, Krisak et al., 1994) (Figure 6). In summary, five different MAP kinase pathways with several functions (e.g. mating, sporulation, cell wall integrity) are described in S. cerevisiae.

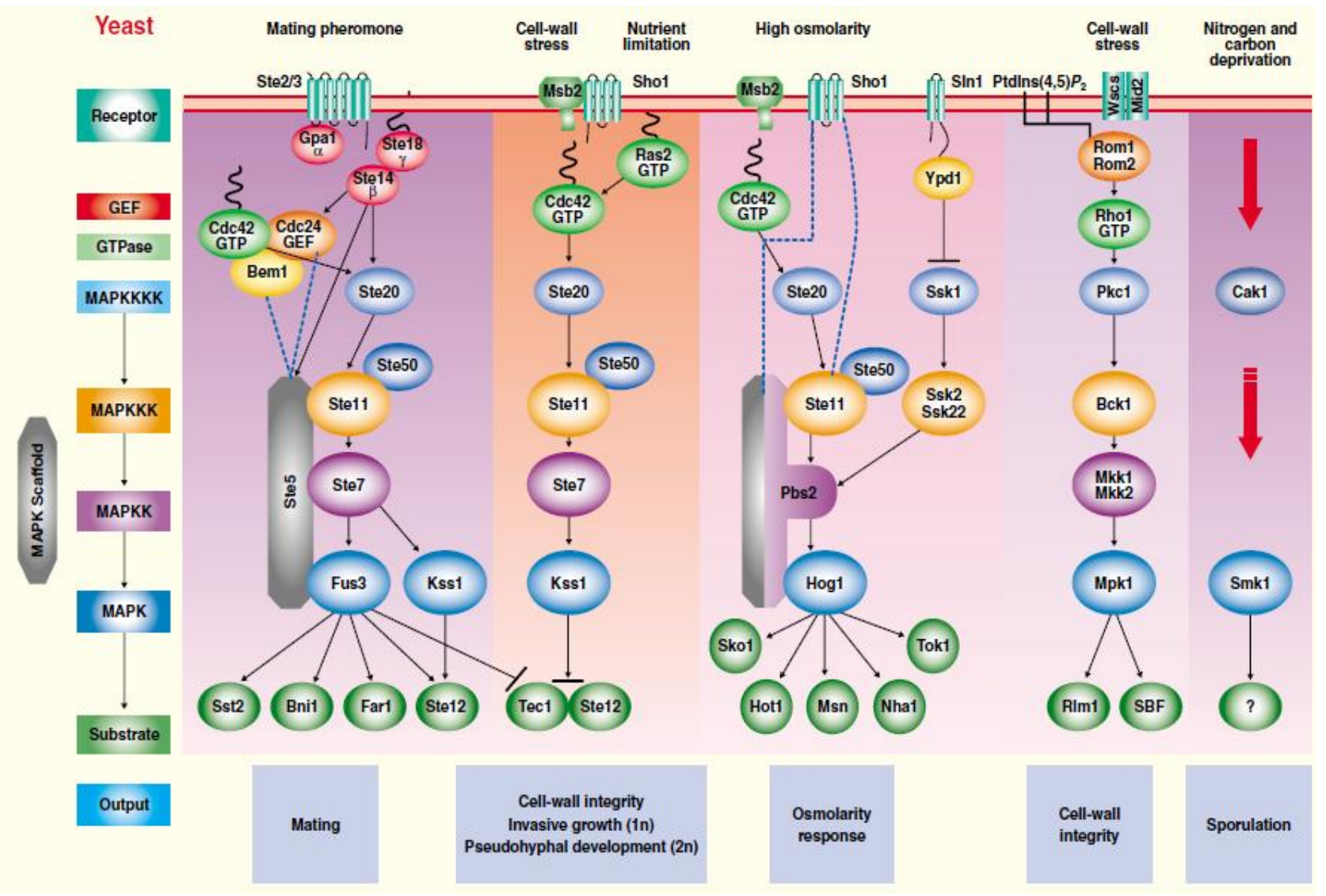

Figure 6: Model of the five MAP kinase pathways regulating mating, filamentation, cell wall integrity, response to high osmolarity and ascospore formation in Saccharomyces cerevisiae (Qi \& Elion, 2005).

\subsubsection{MAP kinase cascades in filamentous fungi}

In filamentous fungi such as $N$. crassa, which undergo highly complex and multicellular developmental phases the situation is much less clear. Based on sequence homologies, three 
basic MAPK modules, resembling one p38-type and two ERK-type kinase cascades have been identified in all sequenced filamentous ascomycetes (Borkovich et al., 2004, Galagan et al., 2005a, Galagan et al., 2005b).

The p38-like MAP kinase pathway (homologous to the $S$. cerevisiae HOG1 cascade) is responsible for osmostress signaling (Fujimura et al., 2003, Jones et al., 2007, Noguchi et al., 2007, Zhang et al., 2002), while the MAK2 ERK-type kinase cascades (homologous to the $S$. cerevisiae mating/filamentation pathway) are important for controlling cell to cell fusion events in vegetative cells and for regulating sexual development and fruiting body formation (Fleissner et al., 2009a, Kothe \& Free, 1998, Li et al., 2005, Maerz et al., 2008, Pandey et al., 2004, Wei et al., 2003). Knowledge about the third MAK1 signaling pathway (homologous to the $S$. cerevisiae cell wall integrity pathway) is quite limited, but mutations in components of this pathway display altered cell walls and autolysis in some areas of colonies (Borkovich et al., 2004, Maerz et al., 2008). Comparative characterization analysis provided evidences that MAPKs act as three distinct modules during vegetative growth and asexual development, but also that the combined activity of all three pathways is required for the formation of complex multicellular fruiting bodies necessary to undergo sexual development (Maerz et al., 2008).

In N. crassa osmostress signaling is conducted through the OS1 histidine kinase to the OS4, OS5 and OS2 p38-like MAP kinase cascade. Mutations in the os genes cause the inability of growing on high osmolarity media and the mutants are resistant to phenylpyrrole fungicides. Also lysis and increased pigmentation of asexually derived spores (macroconidia) and female sterility due to the lack of protoperithecia has been reported, but the cellular or developmental defects have not been analyzed in detail (Fujimura et al., 2003, Jones et al., 2007, Noguchi et al., 2007, Zhang et al., 2002).

In $A$. nidulans, HOG pathway mutants are similarly growth-inhibited under high osmolarity conditions and are sensitive to oxidative stress (Furukawa et al., 2005, Kawasaki et al., 2002). In contrast to Aspergillus nidulans and Neurospora crassa where the activation of the HOG pathway only depends on a two component signaling pathway (Furukawa et al., 2005, Noguchi et al., 2007), the yeast HOG1 cascade depends on two upstream osmosensing branches (the $\operatorname{Sln} 1 p$ transmembrane hybrid-type histidine kinase and a putative seven transmembrane osmosensor kinase (Maeda et al., 1995, Posas et al., 1998a, Posas et al., 1998b). 
Several MAPK components homologous to the yeast pheromone/filamentation pathway have been found in N. crassa. The triple MAP kinase NRC1 was first identified as a repressor of conidiation, but later it was shown that NRC1 is also involved in hyphal fusion and in the activation of the ERK-type MAPK MAK2 (Kothe \& Free, 1998, Li et al., 2005, Pandey et al., 2004).

Mutations in the mak-2 gene and the $p p-1$ gene (the downstream transcription factor homologous to yeast Ste12p that is activated by the MAPK Fus3p/Kss1p) display reduced growth rates, the inability to perform hyphal fusion, shortened aerial hyphae formation and derepressed conidiation. In addition to these phenotypical characteristics, they are also unable to develop protoperithecia, and ascospores are autonomous lethal (Li et al., 2005, Pandey et al., 2004). A similar pleiotrophic phenotype has been observed in SteC MAPKKK mutants in $A$. nidulans, which result in reduced growth rates, more branched hyphae, altered conidiophore morphology, inhibition of heterokaryon formation and inhibited sexual development (Wei et al., 2003).

Information about the third MAP kinase pathway in filamentous fungi is still limited. Mutations in the MAPK homologous to yeast Slt2 $p$ have been generated in A. nidulans, and in several phytopathogenic fungi and displayed altered cell walls and defects in conidial germination and autolysis in central areas of the colony (Bussink \& Osmani, 1999, Hou et al., 2002, Kojima et al., 2002, Mey et al., 2002, Xu et al., 1998). Also the Fusarium graminearum Slt2 homolog MGV1 is required for female fertility, heterokaryon formation and plant infection (Hou et al., 2002).

Summarizing, also in filamentous fungi the presence of three MAP kinase cascades suggested a partial overlap for maintaining the functions of the cell wall and during hyphal fusions, based on shared phenotypes and similar sensitivities against cell wall drugs which distinguished the two ERK-type MAPK routes from the p38-type osmosensing pathway (Figure 7).

\subsubsection{MAP kinase modules in higher eukaryotes}

In contrast to the MAP kinase pathways in yeast or filamentous fungi, mammalian MAPK pathways are difficult to define because they harbor many different cell lines, tissue types and functional redundancy. By genetic analysis, at least five families of MAPKs have been 
described in mammalian cells in Drosophila melanogaster and Caenorhabditis elegans: extracellular signal-regulated kinases (ERK1 and ERK2), Jun N-terminal kinases (JNK1, JNK2 and JNK3); p38 kinase isozymes (p38_, p38_, p38_ and p38_); ERK3/ERK4; and ERK5 (Chang \& Karin, 2001, Chen et al., 2001, Johnson \& Lapadat, 2002, Roux \& Blenis, 2004, Davis, 2000). The first three, and their activators, play important roles in human diseases and are targets for drug development (English \& Cobb, 2002, Manning \& Davis, 2003). Mammalian MAPK modules associate with numerous scaffold proteins that regulate their activity and localization in various cells (Morrison \& Davis, 2003). The scaffold proteins can bind to other proteins, including phosphatases and cytoskeletal proteins, but their significance is not fully defined.

In summary, the MAP kinase modules with their kinases and the corresponding essential scaffold proteins are well understood in yeast whereas the mammalian MAP kinase pathways are more complicated due to the numerous kinases, tissue types and cell lines. Moreover, MAP kinase pathways were also found in filamentous fungi, but in contrast to yeast and mammals, the role of possible scaffold proteins is not yet assigned. For example no homologue to the yeast scaffolding protein Ste5p could be identified in N. crassa.

\subsection{Cross-communication between NDR kinase signaling, PKA and MAP kinases}

Genetic data indicate functional interactions between NDR kinases, proteins of the two ERKtype MAP kinase cascades and protein kinase A (PKA). A connection between Ste20/PAK (= MAPKKKK) and NDR kinase signaling was provided through the analysis of the Schizosaccharomyces pombe NDR kinase mutant orb-6 (Verde et al., 1998). The NDR kinase SpORB6 and the Ste20 kinase SpPAK1 share similar deletion phenotypes, double mutants are synthetically lethal, and the overexpression of SpORB6 in pak-1 partially suppressed the pak1 defect, suggesting that PAK1 acts upstream of ORB6.

Members of the MST2 and MST3 groups of the GC kinases have recently been described as upstream regulators of NDR kinases in yeasts and higher eukaryotes (Emoto et al., 2006, Kanai et al., 2005, Nelson et al., 2003, Stegert et al., 2005). Similarly, the N. crassa GC kinase POD6 and the NDR kinase COT1 are essential for hyphal tip extension and coordinated branch formation, and both kinases have been shown to interact in co-precipitation experiments (Figure 5). Moreover, both cot-1 and pod-6 defects are partially suppressed by 
various environmental stresses, which have been shown to decrease PKA activity and thus bypass the requirement of functional COT1 or POD6 (Gorovits \& Yarden, 2003, Seiler et al., 2006). Thus, COT1/POD6 and PKA may act in parallel pathways to regulate cell polarity in a positive and negative manner, respectively. In addition, mutant of the mak-2 MAPK pathway suppress cot-1 phenotypes along with a concomitant reduction in PKA activity (Maerz et al., 2008). Furthermore, loss of COT1 function resulted in increased MAK1 activity, suggesting COT1 as a potential negative regulator of MAK1 (Maerz et al., 2008, Vogt \& Seiler, 2008). This potential inhibitory COT1-MAK1 connection is strengthened by Enomoto et al., 2008, in which mammalian NDR kinase has been identified as a direct interactor and a negative regulator of JNK pathway MAPKKK MEKK1.

In summary, all these data suggest a signaling network consisting of PKA, MAP kinases and NDR signaling that regulate polar tip extension, cell fusion and sexual development (Figure 7).

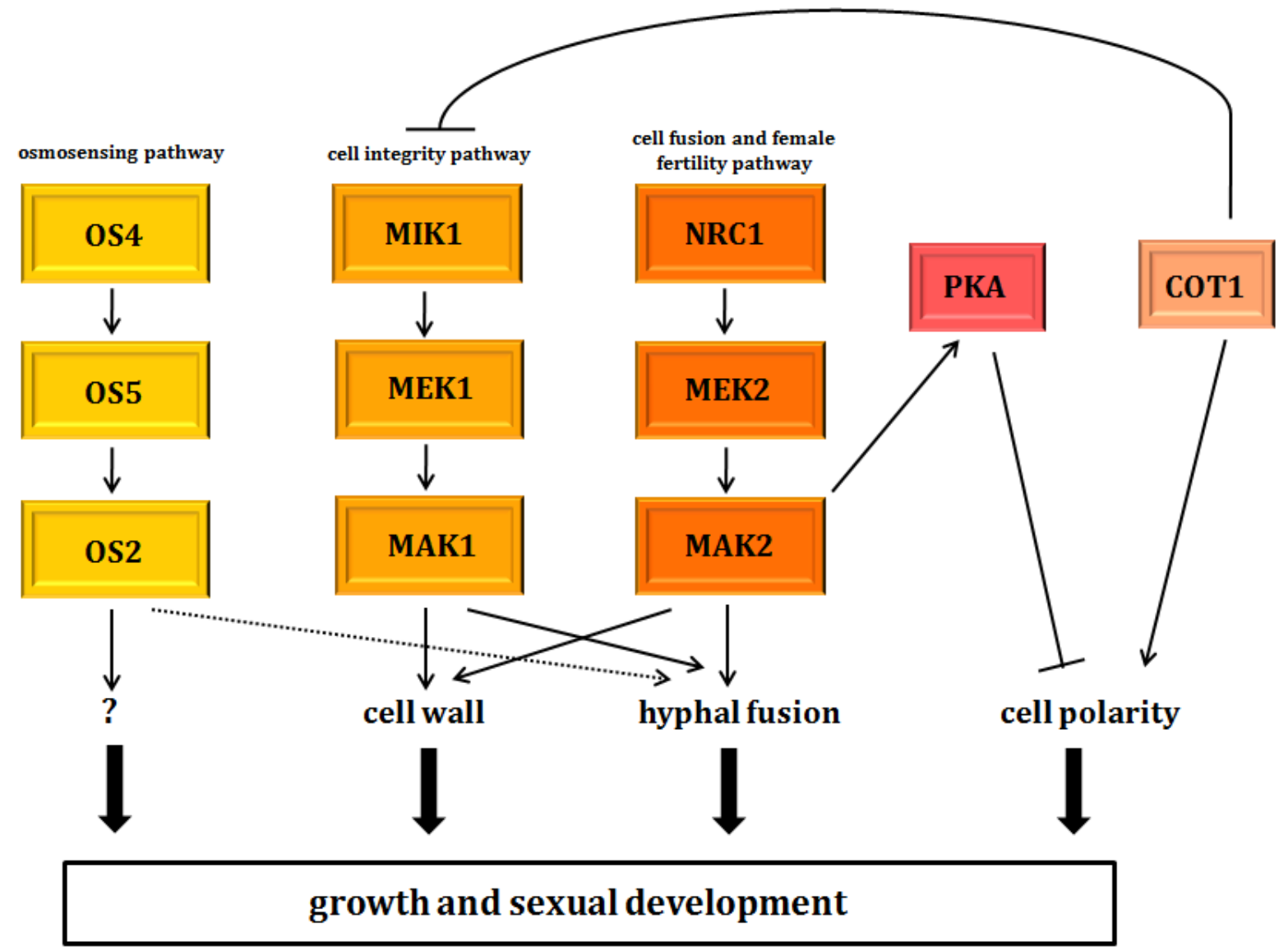

Figure 7: Model of the three MAP kinase modules in Neurospora crassa and the cross-communication between COT1, MAP kinase cascade and PKA signaling pathway (modified from Maerz et al., 2008). 


\subsection{Scaffolding proteins for signal specificity}

Scaffold proteins are thought to generate specificity of signaling pathways to stabilize weak or transient interactions between kinases of a single cascade. Furthermore, scaffolds may catalyze the activation of the cascade components and link associated components e.g. kinases, receptors, G proteins or other signaling parts (Burack \& Shaw, 2000, Elion, 1998, Malleshaiah et al., 2010, Saito, 2010, Schaeffer \& Weber, 1999, Seeliger \& Kuriyan, 2009, Whitmarsh \& Davis, 1998).

\subsubsection{The scaffold protein Ste5p controls mating in budding yeast}

The budding yeast Saccharomyces cerevisiae selects mating partners by interpreting the concentrations of pheromones secreted by potential mates through a network of MAP kinases (MAPK) (Malleshaiah et al., 2010). The first identified and described MAP kinase scaffold was the yeast Ste5p protein (Choi et al., 1994, Kranz et al., 1994, Marcus et al., 1994, Printen \& Sprague, 1994).

Ste5p serves as a hub that brings the kinases of the MAP kinase cascade in a close proximity (Seeliger \& Kuriyan, 2009). However, genetic approaches indicated that Ste5p function could not be defined in linear terms in a signal pathway (Elion, 1998, Kranz et al., 1994). But these studies suggested that Ste5p plays a role in signaling and functions at multiple steps in the cascade to activate Fus3p, the MAPK.

The Ste $5 p$ protein contains distinct structural motifs. At the $\mathrm{N}$-terminus of the protein a membrane anchoring motif (NLS), a RING-H2 Zn finger domain (termed RING or LIM domain), and a cryptic $\mathrm{PH}$ (pleckstrin homology) domain, important for Ste5p homodimerization, Ste11p association, interaction with the MAPK Fus3p and Ste4p G protein binding, were found (Borden, 2000, Choi et al., 1994, Kranz et al., 1994, Whiteway et al., 1995, Winters et al., 2005, Yablonski et al., 1996). The C-terminus of Ste5p contains a vWA (von Willebrand type A) module which is involved in the association with Ste7p (Good et al., 2009). Distinct regions of Ste5p interact with the MAPK Fus3p, the MAPK kinase (MAPKK) Ste7p and the MAPKK kinase (MAPKKK) Ste11p to form the active complex and further with Ste4p, the MAPKKKK (Figure 8A). 
Summarizing, the Ste5p scaffold plays two roles in the mating signal transduction pathway. First, it binds the components of the MAPK cascade and brings them together in an active complex. Second, it associates with the plasma membrane, bringing the kinases to the plasma membrane where the triple MAP kinase Ste11p can be activated by the Ste20p kinase (Bardwell, 2005) (Figure 8B).

So far, it is not clear how the signal transduction from an upstream signal to the MAP kinase cascade occurs in N. crassa.

A

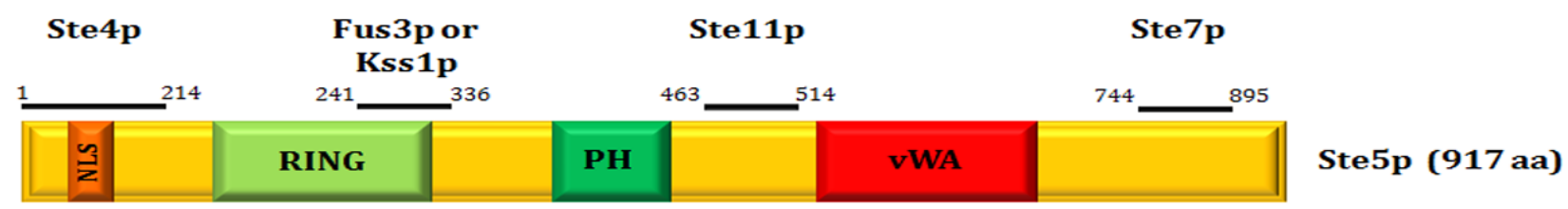

B

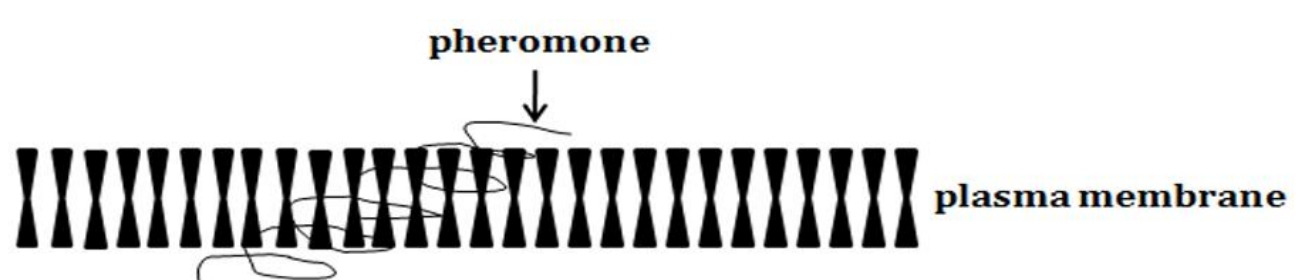

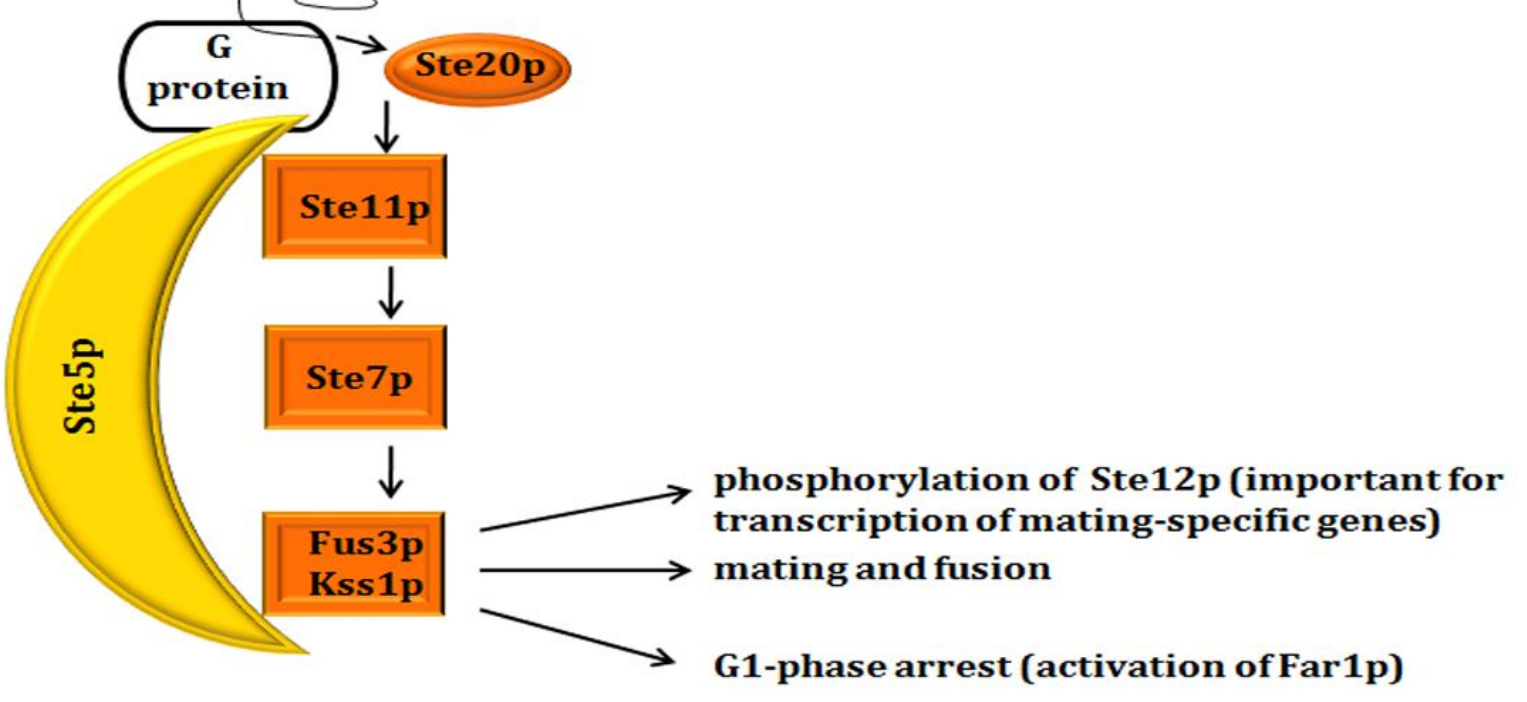

Figure 8: The Ste5p scaffold protein.

(A) Schematic representation of the structural protein organization of Ste5p including the interaction domains for the MAPKKK Ste11p, MAPKK Ste7p and MAPK Fus3p or Kss1p binding (modified to Bhattacharyya et al. and 2006, Cote et al., 2011).

(B) Ste5p-dependent complex in mediating signal transduction during mating (modified to Elion, 1995); for details see text). 


\subsubsection{Members of the MO25 protein family acting as master regulators of Ste20 kinases}

MO25 (mouse protein-25) was first identified as a highly conserved protein expressed at the early cleavage stages of mouse embryogenesis (Miyamoto et al., 1993, Nozaki et al., 1996). Further studies demonstrated that $\mathrm{MO} 25$ act as a scaffolding protein required for activation and stabilization of the LKB1 tumour suppressor protein kinase complex, which functions as an upstream activator of AMPK (AMP-activated protein kinase) protein kinases (Boudeau et al., 2003c, Boudeau et al., 2004). The active LKB1 protein kinase complex is a heterotrimer, consisting of MO25 binding to LKB1 and the pseudokinase STRAD of the GCK family (Baas et al., 2003, Boudeau et al., 2003a, Boudeau et al., 2006, Boudeau et al., 2004). By crystallographic approaches an extensive web of interactions between the $\mathrm{N}$-lobe and the helix $\alpha C$ of STRAD $\alpha$ and the concave surface of MO25 $\alpha$ was identified (Zeqiraj et al., 2009a, Zeqiraj et al., 2009b). Additionally, STRAD $\alpha$ exhibited a C-terminal WEF motif binding to a pocket at the C-terminus convex surface of MO25a (Boudeau et al., 2003a, Boudeau et al., 2003b, Boudeau et al., 2003c, Milburn et al., 2004). Cooperative interactions with MO25a and ATP force STRAD $\alpha$ to adopt a closed confirmation resembling that of an active protein kinase which is required for binding and activating LKB1 (Boudeau et al., 2003a, Boudeau et al., 2003b, Boudeau et al., 2003c, Milburn et al., 2004, Zeqiraj et al., 2009a, Zeqiraj et al., 2009b). In a recent publication, MO25 was described as a master regulator of GCKs by interacting with multiple germinal centre kinases (Filippi et al., 2011).

In the budding yeast $S$. cerevisiae, three kinases (Elm1p, Pak1p and Tos3p) resemble LKB1 and act as upstream kinases to activate the AMPK orthologue SNF1 (Elbing et al., 2006a, Elbing et al., 2006b, Hedbacker \& Carlson, 2008). There are no obvious orthologues of a pseudokinase resembling STRAD suggesting that Elm1p, Pak1p and Tos3p resembling LKB1 are likely regulated in a different manner. The orthologue of $\mathrm{MO25}$, termed Hym1p in $S$. cerevisiae exhibits no interactions with the SNF1 pathway suggesting that Hym1p has other roles in yeast. So, Hym1p was found to interact with an active orthologue of the MST kinase Kic1p (see section 3.2.1.3). Both, Hym1p and Kic1p, regulate transcriptional activity and morphogenesis by activating an NDR kinase termed Cbk1p (Bidlingmaier et al., 2001, Nelson et al., 2003). Also in S. pombe, Pmo25 controls morphogenesis by interacting with an MST kinase Nak1 and regulating the activation of the NDR kinase Orb6 (Kanai et al., 2005, Mendoza et al., 2005). In recent studies, it has been shown that Pmo25 has an additional role in regulating cell separation by interacting with another MST kinase, Ppk11 (Goshima et 
al., 2010). Also in N. crassa, the MO25 orthologue HYM1 interacts with the MST kinase POD6 and the corresponding NDR kinase COT1 (unpublished data). In addition, Karos and Fischer identified hymA in Aspergillus nidulans (1999), deletion of this gene causes an effect on conidiophore development at the metula stage. They also constructed a chimeric protein comprised of the $\mathrm{N}$-terminal half of the $A$. nidulans and the $\mathrm{C}$-terminal half of the mouse homologue MO25. This hybrid protein could fully substitute for hymA function in $A$. nidulans. In addition, the mouse protein itself partially rescued the hymA mutation in the fungus suggesting an evolutionary conservation of this protein from yeast over filamentous fungi to mammals.

In summary, members of the MO25 protein family are 30-40 kDa proteins and are highly conserved. This protein family acts as an important regulator of cell polarity in yeast, filamentous fungi and higher eukaryotes. They acting as adaptor proteins between germinal centre kinases and their potential effector kinases and further as master regulators of GCKs.

\subsubsection{Striatin proteins acting as scaffold proteins and thereby establishing a platform for various protein protein interactions in eukaryotes}

Striatin proteins belong to a protein family which is highly conserved from eukaryotes to mammals, but are absent in budding yeast and plants (Benoist et al., 2006). They are multidomain molecules containing several protein-interacting motifs. MOB3, an identified interaction partner of striatin belongs to the MOB protein family which functions as adaptor proteins of NDR kinases suggesting a possible cross-communication between the NDR kinase signaling and the striatin complex (see section 3.2.1.3). Further, different publications indicate a negative regulation of the Hippo pathway by the STRIPAK complex in Drosophila melanogaster (Kean et al., 2011, Ribeiro et al., 2010).

In mammals, the striatin family consists of three scaffolding proteins composed of striatin, SG2NA and zinedin (Gaillard et al., 2006). These proteins are mainly expressed in the cytoplasm of neurons of the central and the peripheral nervous system (Blondeau et al., 2003, Castets et al., 1996, Castets et al., 2000).

The first member of this protein family, striatin, was first purified from rat brain synaptosomes and consists of 780 amino acids. Due to the expression in neurons with high levels of protein and transcript expression in the striatum, the protein was named striatin. The second protein of the striatin family, SG2NA (S/G2 „nuclear antigen“), was originally 
identified as a nuclear protein with cell-cycle regulated expression, but recent experiments showed that SG2NA is an exclusively cytosolic and membrane-bound protein (Baillat et al., 2001, Castets et al., 2000, Lu et al., 2004). SG2NA occurs in different isoforms due to alternative splicing. In addition to the major isoforms, SG2NA $\alpha$ with 713 aa and SG2NA $\beta$ with 797 aa, Sanghamitra et al., 2008 published the existence of additional five isoforms dependent on the expression in different tissues and developmental stages.

Zinedin, the third protein of the striatin family in mammals, was identified on the basis of sequence similarities with striatin and SG2NA and consists of 753 aa (Castets et al., 2000).

All proteins of the striatin family share four identical protein-protein interaction domains: from the $\mathrm{N}$ - to the $\mathrm{C}$-terminus, a caveolin-binding domain, a coiled-coil structure, a $\mathrm{Ca}^{2+}$ calmodulin-binding domain and a WD-repeat domain (Bartoli et al., 1998, Gaillard et al., 2006, Gaillard et al., 2001) (Figure 9).

All caveolin partners possess a caveolin-binding consensus " $\operatorname{x} \Phi \times x x x \times \times x \times \Phi^{\prime \prime}(\Phi$ corresponding to aromatic aa and $x$ to any aa) (Bartoli et al., 1998, Couet et al., 1997a, Couet et al., 1997b, Gaillard et al., 2006, Gaillard et al., 2001). Striatin, SG2NA and zinedin can directly bind caveolin-1 shown by pull-down experiments and their interaction can be inhibited in the presence of $\mathrm{Ca}^{2+}$ (Gaillard et al., 2001). Additionally, Petralia et al., 2003 demonstrated that caveolins are concentrated within dendritic spines of hippocampal neurons suggesting a potential localization of striatin proteins in membranous microdomains of dendritic spines through their interaction with $\mathrm{Ca}^{2+}$-regulated caveolin (Benoist et al., 2006).

The second highly conserved coiled-coil domain with $94 \%$ identity among the striatin family members seems to be necessary for the homo- and hetero-oligomerization of these proteins. This oligomerization step is important for correct localization to the dendritic spines (Gaillard et al., 2006).

The third domain structure in this protein family is called $\mathrm{Ca}^{2+}-\mathrm{CaM}$-binding domain and the interaction between Calmodulin and the striatin members are connected through an amphipathic helix (Bartoli et al., 1998, Castets et al., 1996, Castets et al., 2000). Calmodulin consists of a helix-loop-helix structure and is able to bind $\mathrm{Ca}^{2+}$ which mediates a change in the conformation of Calmodulin to facilitate protein-protein interactions and the regulation of different CaM-dependent proteins e.g. CaM-dependent kinases and the adenylate cyclase which are involved in various biological processes like apoptosis, metabolism, muscle 
contraction, the cellular mechanisms of learning and memory and immune response (Chou et al., 2001, Colomer \& Means, 2007, Wayman et al., 2008, Witczak et al., 2008).

In the C-terminus of the striatin proteins 8 putative WD-repeat motifs were determined (Smith et al., 1999). The WD-repeat superfamily is involved in cytoskeletal assembly and vesicular traffic. The WD-repeat propeller structure forms a stable platform for different reversible protein-protein interactions (Lambright et al., 1996, Sondek et al., 1996).

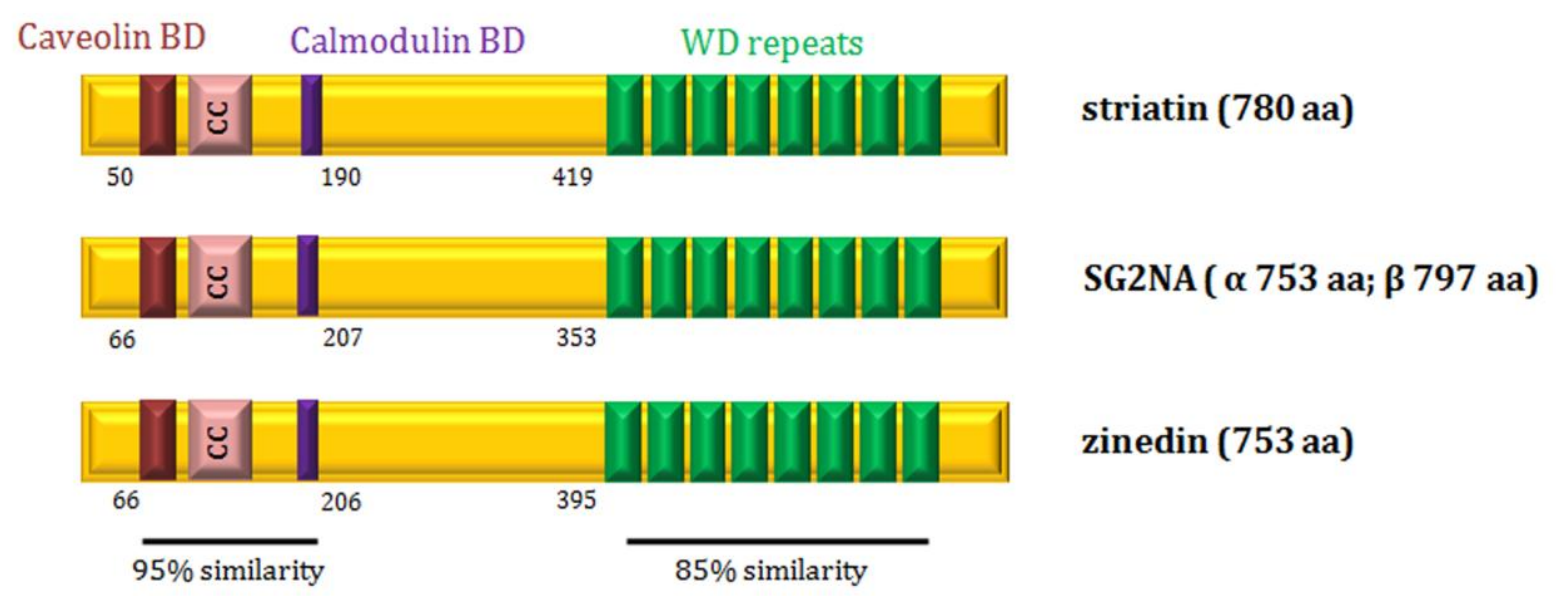

Figure 9: Domain structure of the striatin proteins in mammals. All three proteins exhibit the same proteinprotein interaction domains from $\mathbf{N}$ to $\mathbf{C}$-terminus: caveolin-binding domain, coiled-coil domain, calmodulinbinding domain and the 8 WD repeats (modified to Benoist et al., 2006).

So far, seven homologues of the striatin family were described in filamentous fungi and animals, Pro11 in the homothallic ascomycete Sordaria macrospora, HAM3 in the heterothallic ascomycete Neurospora crassa, Fsr1 in the plant pathogen fungi Fusarium verticillioides and Fusarium graminearum, StrA in Aspergillus nidulans, CKA in Drosophila melanogaster and the two isoforms SG2NA $\alpha$ und SG2NAa+ in Carassius auratus (Chen et al., 2002, Ma et al., 2009, Poeggeler \& Kueck, 2004, Shim et al., 2006, Simonin et al., 2010, Wang et al., 2010, Yamamura \& Shim, 2008) (Figure 9).

The striatin homologue Pro11 of the filamentous ascomycete Sordaria macrospora has a crucial role in fruiting body development (Bernhards \& Poeggeler, 2011). Surprisingly, the sterile phenotype of a pro11 mutant was complemented by a mouse striatin homologue indicating an evolutionarily conservation of the striatin proteins from fungi to humans (Poeggeler \& Kueck, 2004).

Also in $N$. crassa, the $\Delta$ ham-3 strain shows developmental defects in protoperithecia formation and cell fusion which supports the S. macrospora data (Simonin et al., 2010). 
The protein Fsr1 in Fusarium species shares 60\% sequence identity with the Sordaria macrospora Pro11 and plays a regulatory role in cell differentiation and ascocarp development. Moreover, published data by Shim et al., 2006 showed that FSR1 is essential for female fertility and virulence in F. verticillioides (Figure 10).

Furthermore, in Aspergillus nidulans, $\Delta$ strA strains show multiple defects in conidium germination, mycelial radial growth, production of diffusible red pigment, and reduced conidiation. The most striking phenotype is the production of abnormally small cleistothecia that are defective in ascosporogenesis (Wang et al., 2010).

In summary, striatin proteins are also conserved in most filamentous fungi and play a crucial role during sexual development (e.g. correct fruiting body and protoperithecia formation).

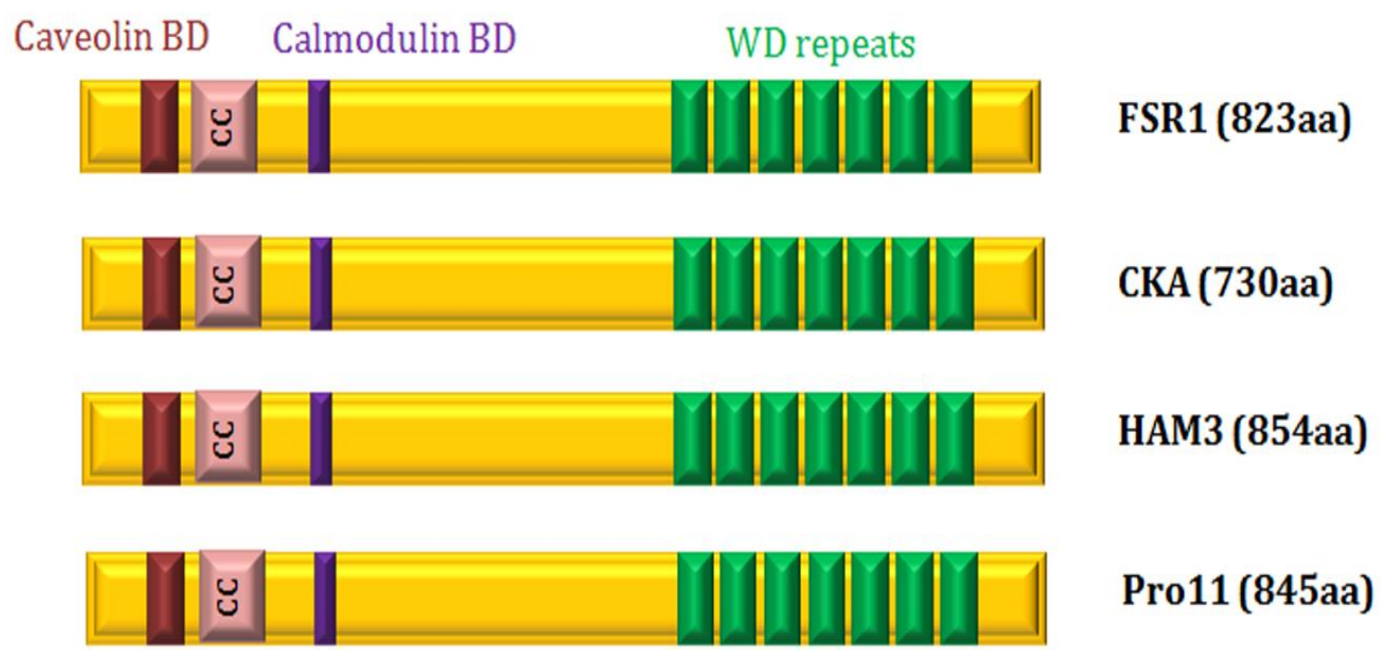

Figure 10: Domain structure of striatin proteins in filamentous fungi (modified to Chen \& Lin, 2001, Poeggeler \& Kueck, 2004, Shim et al., 2006, Simonin et al., 2010). The order of interaction domains from N to Cterminus: caveolin-binding domain, coiled-coil domain, calmodulin-binding domain and the 7 WD repeats.

\subsubsection{Functions of striatin proteins}

The physiological roles of the striatin family members are beginning to emerge. So far, the striatin members play important roles in various pathways, and their misregulation causes developmental impairment. The deletion of the striatin homologues FSR1 in $F$. graminearum, HAM3 in $N$. crassa and StrA in A. nidulans lead to the loss of fertility. Additionally, the fungus $N$. crassa is not able to perform hyphal cell fusion and also the pro11 mutant in S. macrospora is not able to form sexual structures (Poeggeler \& Kueck, 2004, Shim et al., 2006, Simonin et al., 2010, Wang et al., 2010).

Striatin members also play crucial roles in the development in mammalian eukaryotes and are critical for the correct development of motoneurons (Bartoli et al., 1999). Furthermore, 
striatin is essential for the building of dendritic arborization, probably by calcium-regulated mechanisms, since calcium signaling is required for proper neuronal maturation (Benoist et al., 2006, Jourdain et al., 2003). Bartoli et al. published in 1999 that the downregulation of striatin in the brain of rats maintain disturbance in locomotive activity and a reduction of the growth of dendritic spines.

In the fruit fly D. melanogaster, the absence of the striatin homologue CKA induces a dorsalopen cuticle phenotype and death (Chen et al., 2002, Martin \& Wood, 2002). The embryos display head defects and lack the dorsal epidermis indicated by a large dorsal hole in the cuticle.

\subsubsection{Potential interaction partners of striatin}

The first interaction protein of striatin was Phocein/MOB3 identified by yeast two-hybrid studies in Rattus norvegicus (Baillat et al., 2001). Phocein/MOB3 is widely expressed, consists of 225 aa and displays the same localization pattern in somato-dendritic compartments in different neurons, especially in dendritic spines, like the striatin family members. This protein belongs to the Mob protein family, which is characterized by the Mob domain (Baillat et al., 2001, Luca \& Winey, 1998, Ponchon et al., 2004). Basic facts about the Mob protein family including the phocein subgroup are summarized in chapter 3.2.1.3. In addition to the interaction with striatin proteins, phocein also interact with Eps15 („epidermal growth factor receptor substrate 15“), a nucleoside-diphosphate kinase and dynamin-1. All these proteins are involved in endocytosis and vesicular trafficking. Therefore striatin proteins associated with phocein may also act in the process of trafficking within dendritic spines.

Protein phosphatase A (PP2A) was identified as a second potential interaction partner of the striatin family in mammals. Moreno et al., 2000 has shown by biochemical approaches that striatin and SG2NA acting as subunit $\mathrm{B}^{\prime}$ or $\mathrm{B}^{\prime \prime}$ of PP2A in the absence of PP2A B. Further the inhibition of PP2A by okadaic acid increases striatin and SG2NA phosphorylation suggesting that both proteins are substrates of protein phosphatase A (Moreno et al., 2001). In recent studies, other striatin interacting proteins were identified in the STRIPAK pathway („,striatininteracting phosphatase and kinase“ pathway) (Goudreault et al., 2009). Phocein/Mob3, STRIP1 and STRIP2 (,striatin-interacting proteins 1 und 2“), CCM3 („,cerebral cavernous malformation $3^{\prime \prime}$ ) and all members of GCKIII of the Ste20 kinase protein family belong to 
STRIPAK. So far, the regulation of the STRIPAK complex is unknown and beginning to emerge, but it seems that all mentioned proteins acting together in a multiprotein signaling complex which regulates different signal cascades (Goudreault et al., 2009, Kean et al., 2011) (Figure 11).

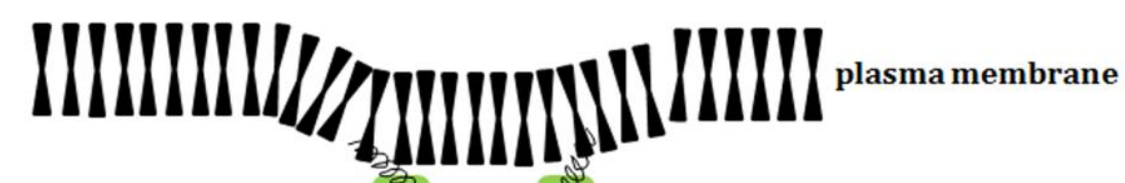

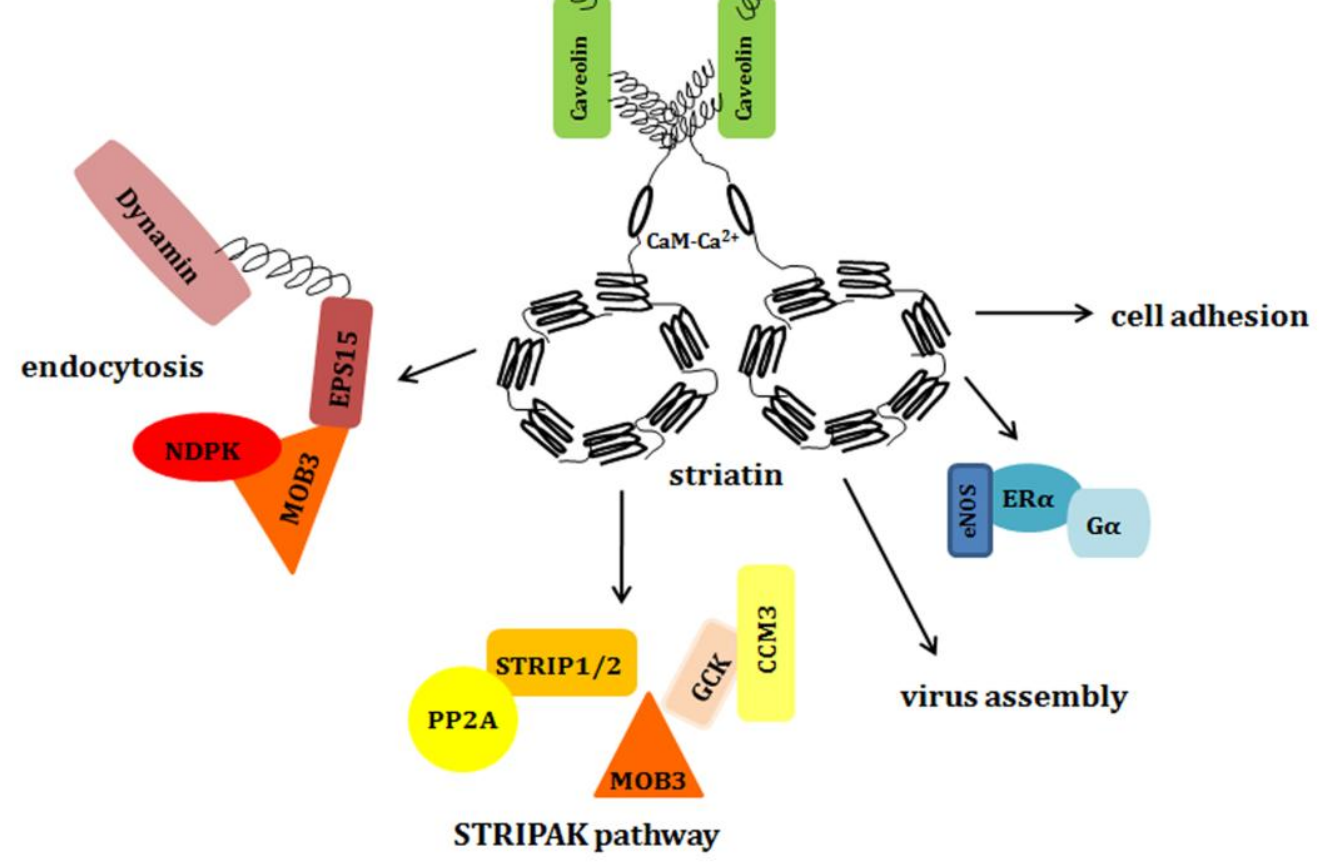

Figure 11: Overview about the interacting pathway of striatin (modified to Benoist et al., 2006). For further details see text.

\subsection{Aim of this work}

The NDR kinase COT1 is essential for hyphal elongation in the filamentous fungus Neurospora crassa. Genetic and biochemical researches have facilitated the identification of an intricate signaling network consisting of PKA, the MAK2 MAP kinase cascade and the COT1 complex that regulate polar tip growth, cell fusion and sexual development. Our hypothesis is that multiple functional connections exist between COT1 signaling and MAP kinase cascades and that the COT1-POD6 interacting protein HYM1 may act as a scaffold 
connecting these pathways. These suggestions should be verified by different approaches (yeast two-hybrid analysis, co-immunoprecipitation and localization studies).

On the morphological level, differences and similarities between the deletion strains of the COT1 pathway and the MAP kinase cascade could be observed indicating the involvement of another complex, the striatin complex. Striatin proteins act as scaffolding proteins in filamentous fungi and higher eukaryotes. On the one hand, this work should clarify the genetic connection between the COT1 pathway and the MAP kinase cascade by the potential linker protein HYM1. Additionally, the search of potential upstream regulators of the MAK2 kinase cascade is a subject of investigation due to the fact that no homologue of the yeast MAP kinase scaffold protein Ste5p is known in $N$. crassa. On the other hand, the link between the striatin complex and the MAK2 kinase cascade should be analyzed.

Moreover, this work should also clarify how the NDR kinase COT1 is activated and carried to its effective location. 


\section{Materials and Methods}

\subsection{Media and growth conditions}

Standard chemicals and culture media components in this study were used from AppliChem $\mathrm{GmbH}$, Carl Roth GmbH \& Co. KG, Invitrogen GmbH, Merck KGaA, Oxoid Deutschland GmbH, Roche Diagnostics $\mathrm{GmbH}$, SERVA Electrophoresis $\mathrm{GmbH}$ and Sigma-Aldrich Chemie $\mathrm{GmbH}$.

General procedures for growth and genetic procedures of Neurospora crassa are available in the Fungal Genetics Stock Center at www.fgsc.net or are described in (Davis, 1970).

Escherichia coli $\mathrm{DH} 5 \alpha$ were grown at $37^{\circ} \mathrm{C}$ in liquid LB medium (1\% tryptone, $0.5 \%$ yeast extract, $1 \% \mathrm{NaCl}$ ) or on solid medium (with 1.5\% agar) modified from (Bertani, 1951). For selection media $100 \mu \mathrm{g} / \mathrm{ml}$ ampicillin or $50 \mu \mathrm{g} / \mathrm{ml}$ kanamycin were added (all from SigmaAldrich, Taufkirchen, Germany). The cultures were incubated at $37^{\circ} \mathrm{C}$.

Saccharomyces cerevisiae cultures were grown at $30^{\circ} \mathrm{C}$ under non-selective conditions in Yeast Extract Peptone Dextrose (YEPD) medium (2\% pepton, 1\% yeast extract, 2\% glucose, for solid medium 2\% agar was added) or in Synthetic Defined (SD) minimal medium lacking different amino acids for selection of plasmid expression or interaction in the yeast twohybrid assay $(0.17 \%$ yeast nitrogen base (w/o amino acids, w/o ammonium sulphate), $0.5 \%$ ammonium sulphate, $10 \mathrm{mg}$ L-adenine sulphate, $10 \mathrm{mg}$ L-argenine, $10 \mathrm{mg}$ L-histidine, $15 \mathrm{mg} \mathrm{L}$ isoleucine, $50 \mathrm{mg}$ L-leucine, $15 \mathrm{mg}$ L-lysine, $10 \mathrm{mg}$ L-methionine, $25 \mathrm{mg}$ L-phenylalanine, $100 \mathrm{mg}$ L-threonine, $10 \mathrm{mg}$ L-tryptophane, $15 \mathrm{mg}$ L-tyrosine, $10 \mathrm{mg}$ uracil and $75 \mathrm{mg}$ L-valine, $2 \%$ glucose, $2 \%$ agar)(see also section 4.6 ).

Neurospora crassa strains were cultured in liquid Vogel's minimal medium (VMM) with 2\% sucrose or on solid (supplemented with $2 \%$ agar) (Vogel, 1956, Vogel, 1964). For colonial growth of the fungus sucrose was replaced by $2 \%$ L-sorbose, $0.05 \%$ glucose and $0.05 \%$ fructose (Mishra \& Tatum, 1972, Brockman \& de Serres, 1963). For crosses, medium agar plates with $2 \%$ corn meal agar (Sigma, St. Louis, USA) and $0.1 \%$ glucose were used to restrict formation of conidia and induce protoperithecia formation of the female parent. For auxotrophic strains, culture media were supplemented with $8 \mu \mathrm{g} / \mathrm{ml}$ nicotinamide, $200 \mu \mathrm{g} / \mathrm{ml}$ tryptophane and/or $150 \mu \mathrm{g} / \mathrm{ml}$ histidine. $200 \mu \mathrm{g} / \mathrm{ml}$ hygromycin B (InvivoGem, San Diego, USA), $15 \mu \mathrm{g} / \mathrm{ml}$ nourseothricin (Werner BioAgents, Jena, D) and $200 \mu \mathrm{g} / \mathrm{ml}$ glufosinateammonium (Sigma-Aldrich, Taufkirchen, Germany) were added to media for selection of the respective integrated resistance cassettes. Cultures were grown at $37^{\circ} \mathrm{C}$, although temperature sensitive strains were grown at $20-25^{\circ} \mathrm{C}$. Growth tests were performed on solid Vogel's minimal medium at room temperature.

\subsection{Plasmid construction}

\subsubsection{General cloning procedure}

The cloning procedures were planned and documented using the DNASTAR ${ }^{\circledR}$ SeqBuilder (Version 8.0.3(1); DNASTAR, Inc., USA). Sequences of genes were obtained from the Broad Neurospora crassa Database www.broadinstitute.org. 
The plasmid construction started with amplifying the DNA fragments by polymerase chain reaction (PCR) and subcloning into the PJET1.2 blunt vector of the CloneJET ${ }^{\mathrm{TM}}$ PCR Cloning Kit (Fermentas $\mathrm{GmbH}$, Germany). The resulting plasmids were analyzed by restriction digests and sequencing of the inserts. After ligation of inserts into the respective end vectors, plasmids were checked by restriction patterns and final sequencing.

Primers and plasmids used in this study are listed in Table 3 and Table 4, respectively.

Table 3: Primers used in this study. Restriction enzyme recognition sites are bold and underlined, mismatched nucleotides for insertion of mutations are depicted in lower, italic letters.

\begin{tabular}{|c|c|}
\hline Primer name & Sequence \\
\hline AD0772-Ndel5 & CAT ATG GAT GGC CGA CCG CG \\
\hline AD0772-EcoRI3 & GAA TTC CTA ACC ACC ATG CCC \\
\hline AD2393-EcoRI5 & CAG TGA ATT CAT GAG CAG CGC ACA AAG AGGC \\
\hline AD2393-BamHI3 & GAT GGA TCC TCA CCT CAT AAT CTC CTG GTA G \\
\hline AD4202-Ndel5 & CAT ATG TCC AAC CAG GAG CAG AC \\
\hline AD4202-EcoRI3 & GAA TTC TTA CTC GAA GAT CCA GG \\
\hline AD4612-EcoRI5 & CAG TGA ATT CAT GGC CGA CCC ATT TGC CC \\
\hline AD4612-BamHI3 & GAT GGA TCC TCA AAA TCG GCC AGT TGG AGG \\
\hline AD6182-EcoRI5 & CAG TGA ATT CAT GGC CAT GCT GGC ATC CAA \\
\hline AD6182-BamHI3 & GAT GGA TCC CTA TGT CCC GGG CAC AGG CG \\
\hline ADFLAG2393-BamHI5 & GGA TCC ATG AGC AGC GCA CAA A \\
\hline ADFLAG2393-Pacl3 & TTA ATT AAT CAC CTC ATA ATC TCC TG \\
\hline ADFLAG4202-BamHI5 & GGA TCC ATG TCC AAC CAG GAG C \\
\hline ADFLAG4202-Pacl3 & ITA ATT AAT TAC TCG AAG ATC CAG G \\
\hline ADFLAG4612-BamHI5 & GGA TCC ATG GCC GAC CCA TTT \\
\hline ADFLAG4612-Pacl3 & ITA ATT AAT CAA AAT CGG CCA GTT G \\
\hline ADFLAG6182-BamHI5 & GGA TCC ATG GCC ATG CTG GC \\
\hline ADFLAG6182-EcoRI3 & GAA TTC CTA TGT CCC GGG CAC \\
\hline ADFLAG7674-Smal5 & CCC GGG CAT GTC GCT TCC CC \\
\hline ADFLAG7674-EcoRI3 & GAA TTC CTA TGC GAC CTG CTC AG \\
\hline ADHA0772-BamHI5 & GGA TCC ATG GCC GAC CGC G \\
\hline ADHA0772-EcoRI3 & GAA TTC CTA ACC ACC ATG CCC ATC \\
\hline ADHA4202-BamHI5 & GGA TCC ATG TCC AAC CAG GAG C \\
\hline ADHA4202-Pacl3 & ITA ATT AAT TAC TCG AAG ATC CAG G \\
\hline ADHA8741-Smal5 & CCC GGG TAT GGG CAC CAA CG \\
\hline ADHA8741-EcoRI3 & GAA TTC CCTA CCT CGC GTA CAC \\
\hline ADHA8741S2A-MP5 & CAC CGA AGA AAG AGC gcg AGC GGC ATG GCG \\
\hline ADHA8741S2A-MP3 & CGC CAT GCC GCT cgc GCT CTT TCT TCG GTG \\
\hline ADHA8741S2E-MP5 & CAC CGA AGA AAG AGC gag AGC GGC ATG GCG \\
\hline ADHA8741S2E-MP3 & CGC CAT GCC GCT ctc GCT CTT TCT TCG GTG \\
\hline ADMYC3576-SgsI5 & GGC GCG CCT ATG TCA TTC CTC TTT GGG AGA G \\
\hline ADMYC3576-Spel3 & ACT AGT TTA CCG CTG CGG CAC C \\
\hline ADGFP3576-Xbal5 & $\underline{\text { TCT AGA ATG TCA TTC CTC TTT GGG }}$ \\
\hline ADGFP3576-BamHI3 & GGA TCC CCC GCT GCG GCA CC \\
\hline ADGFP8741-Spel5 & ACT AGT ATG GGC ACC AAC GGC \\
\hline
\end{tabular}




\begin{tabular}{|c|c|}
\hline ADGFP8741-Smal3 & CCC GGG CCT CGC GTA CAC TT \\
\hline ADGFP11367-SgsI5 & GC GGC GCG CCC ATG GCT GA \\
\hline ADGFP11376-Pacl3 & AG ITA ATT AAT TAC ATG CCA CCA AG \\
\hline ADGFP0772-Xbal5 & ICT AGA GGA TGG CCG ACC GC \\
\hline ADGFP0772-BamHI3 & GGA TCC CAC CAC CAT GCC C \\
\hline ADGFP4202-Xbal5 & ICT AGA ATG TCC AAC CAG GAG C \\
\hline ADGFP4202-Smal3 & CCC GGG CTC GAA GAT CCA GG \\
\hline ADGFP7296-Xbal5 & TCT AGA ATG GAC AAC ACC AAC CGC \\
\hline ADGFP7296-BamHI3 & GGA TCC CTC GGA AGT TGT TGT CG \\
\hline ADGFP7674-Xbal5 & TCT AGA ATG TCG CTT CCC CCG \\
\hline ADGFP7674-Smal3 & CCC GGG TGC GAC CTG CTC \\
\hline DJ_Cot_Stopp_Xhol3 & CTC GAG CTC GGA ATG TGT TGT CGA AAC \\
\hline DJ_Cot_Start_Xhol5 & CTC GAG ATG GAC AAC ACC AAC CGC \\
\hline DJ_Cot_3UTR_Sacl5 & GAG CTC TTG CGT TAC GTC TTC TCG CTG \\
\hline DJ_Cot_3UTR_Sacl3 & GAG CTC GTT ATA TTA GAA GAA AGT ACT T \\
\hline ADendoGFP3576-Xhol5 & CTC GAG ATG TCA TTC CTC TTT GG \\
\hline ADendoGFP3576-Xhol3 & CTC GAG CCC GCT GCG GCA C \\
\hline ADendoGFP3576-BamHI-3UTR5 & GGA TCC GGT AAC TAA TTG GTC TG \\
\hline ADendoGFP3576-BamHI-3UTR3 & GGA TCC TTT TTT CCT TCT TTT CTC \\
\hline ADpMF272-MP5 & $\begin{array}{l}\text { GGA TCC CCG GGT TAA TTA ACa tcG TGA GCA AGG GCG } \\
\text { AGG AGC }\end{array}$ \\
\hline ADpMF272-MP3 & $\begin{array}{l}\text { GCT CCT CGC CTT GCT CAC gat GTT ATT AAC CCG GGG } \\
\text { ATC C }\end{array}$ \\
\hline AD6182KD-5f & GAA CTG CTC GCC GTG agg CAG GTC GAG ACA CCT G \\
\hline AD6182KD-3r & CAG GTG TCT CGA CCT Gcc tCA CGG CGA GCA GTT C \\
\hline AD4612DA-MutP5 & $\begin{array}{l}\text { GAG AGC TAG TTA ACg atG TCG CCg acG ACT TTG TGG } \\
\text { GTA CAT C }\end{array}$ \\
\hline AD4612DA-MutP3 & $\begin{array}{l}\text { GAT GTA CCC ACA AAG TC } g \text { tcG GCG ACa tcG TTA ACT } \\
\text { AGC TCT C }\end{array}$ \\
\hline AD6182DAPM-MutP5 & $\begin{array}{l}\text { CAG TTC GGT GGG CTG AGA tcg CCT AGC GAG CTT ATC } \\
\text { GCC }\end{array}$ \\
\hline AD6182DAPM-MutP3 & $\begin{array}{l}\text { GGC GAT AAG CTC GCT AGG cga TCT CAG CCC ACC GAA } \\
\text { CTG }\end{array}$ \\
\hline AD6182DADel-MutP5 & GAA TTC GGT CGC ATG AGA GAC TG \\
\hline AD6182DADel-MutP3 & GAA TTC TAC TAC GAC AGC GGC \\
\hline AD4202DN-MutP5 & $\begin{array}{l}\text { GAC ATG GGC CGC AAC GTC tac CAC GGC TCC GAC TCC } \\
\text { GTC GAG }\end{array}$ \\
\hline AD4202DN-MutP3 & $\begin{array}{l}\text { CTC GAC GGA GTC GGA GCC GTG gta GAC GTT GCG GCC } \\
\text { CAT GTC }\end{array}$ \\
\hline PalmCOT1-5-Xbal & $\begin{array}{l}\text { TTG TCT AGA ATG GGC TGC GTC TGC AGC TCT AAC GAC } \\
\text { AAC ACC AAC CGC CCC C }\end{array}$ \\
\hline COT1-3-Apal & TTG GGG CCC TTA TCG GAA GTT GTT GTC G \\
\hline PalmMOB2A-Xbal & $\begin{array}{l}\text { TTG TCT AGA ATG GGC TGC GTC TGC AGC TCT AAC TAC } \\
\text { CCA TAC GAT GTT CCA GAT TAC }\end{array}$ \\
\hline
\end{tabular}


Table 4: Plasmids used in this study. Construction intermediates (DNA fragments inserted into the pJet1.2 blunt for subcloning) are not listed.

\begin{tabular}{|c|c|c|}
\hline Plasmid & Short description & Source \\
\hline pJet1.2 blunt & $\begin{array}{l}\text { Cloning vector for subcloning of PCR } \\
\text { fragments }\end{array}$ & $\begin{array}{l}\text { Fermentas } \mathrm{GmbH} \text {, } \\
\text { Germany }\end{array}$ \\
\hline pGBKT7 & $\begin{array}{l}\text { Yeast two-hybrid vector for expression } \\
\text { of } \mathrm{N} \text {-terminal GAL4 DNA binding } \\
\text { domain fusion proteins under control } \\
\text { of truncated } \mathrm{P}_{\mathrm{ADH} 1} \text {; carrying TRP1 }\end{array}$ & Clontech, USA \\
\hline pGBKT7-53 & pGBKT7; murine p53 $3_{a a 72-390} \mathrm{cDNA}$ & Clontech, USA \\
\hline pGBKT7-Lam & pGBKT7; human lamin C cDNA & Clontech, USA \\
\hline pGBKT7-2393 & pGBKT7; NCU02393 (mak-2) cDNA & this study \\
\hline pGBKT7-4612 & pGBKT7; NCU04612 (mek-2) cDNA & this study \\
\hline pGBKT7-6182 & pGBKT7; NCU06182 (nrc-1) cDNA & this study \\
\hline pGBKT7-4202 & pGBKT7; NCU04202 (ndk-1) cDNA & this study \\
\hline pGBKT7-3576 & pGBKT7; NCU03576 (hym-1) cDNA & kind gift of S. Maerz \\
\hline pGBKT7-0772 & pGBKT7; NCU00772 (gck-1) cDNA & this study \\
\hline pGBKT7-7296 & pGBKT7; NCU07296 (cot-1) cDNA & kind gift of S. Maerz \\
\hline pGADT7 & $\begin{array}{l}\text { Yeast two-hybrid vector for expression } \\
\text { of } \mathrm{N} \text {-terminal GAL4 activation domain } \\
\text { fusion proteins under control of full- } \\
\text { length } \mathrm{P}_{\mathrm{ADH} 1} \text {; carrying } L E U 2\end{array}$ & Clontech, USA \\
\hline pGADT7-T & $\begin{array}{l}\text { pGADT7; SV40 large T-antigen }{ }_{a a 86-708} \\
\text { cDNA }\end{array}$ & Clontech, USA \\
\hline pGADT7-2393 & pGADT7; NCU02393 (mak-2) cDNA & this study \\
\hline pGADT7-4612 & pGADT7; NCU04612 (mek-2) cDNA & this study \\
\hline pGADT7-6182 & pGADT7; NCU06182 (nrc-1) cDNA & this study \\
\hline pGADT7-4202 & pGADT7; NCU04202 (ndk-1) cDNA & this study \\
\hline pGADT7-0772 & pGADT7; NCU0772 (gck-1) cDNA & this study \\
\hline pGADT7-Pro11N & pGADT7; SmPro11aacDNA & kind gift of Y. Bernhards \\
\hline pGADT7-Pro11C & pGADT7; SmPro11aacDNA & kind gift of Y. Bernhards \\
\hline pGADT7-Mob3 & pGADT7; SmMob3cDNA & $\begin{array}{l}\text { kind gift of } Y \text {. Bernhards } \\
\text { from AG Pöggeler }\end{array}$ \\
\hline $\begin{array}{l}\text { pGADT7Rec- } \triangle \mathrm{N}- \\
\text { RanBPM }\end{array}$ & $\begin{array}{l}\text { pGADT7Rec; murine RanBPM } \\
\text { cDa51-654 } \\
\text { cDNA }\end{array}$ & (Tucker et al., 2009) \\
\hline pFLAGN1 & $\begin{array}{l}\text { Fungal expression vector for } \mathrm{N} \text {-terminal } \\
\text { 3xFLAG fusion proteins under control of } \\
\mathrm{P}_{\mathrm{ccg}-1} \text {; target to his-3 locus }\end{array}$ & $\begin{array}{l}\text { (Kawabata \& Inoue, } \\
\text { 2007) }\end{array}$ \\
\hline pFLAGN1-7674 & pFLAGN1; NCU07674 & this study \\
\hline pFLAGN1-4202 & pFLAGN1; NCU04202 & this study \\
\hline pFLAGN1-2393 & pFLAGN1; NCU02393 & this study \\
\hline pFLAGN1-6182 & pFLAGN1; NCU06182 & this study \\
\hline pFLAGN1-6182DAPM & pFLAGN1; NCU06182 ${ }^{\text {P448S }}$ & this study \\
\hline pFLAGN1-6182DADel & pFLAGN1; NCU06182 deletion aa41-590 & this study \\
\hline pFLAGN1-6182 ${ }^{\mathrm{K} 665 \mathrm{D}}$ & pFLAGN1; NCU06182 ${ }^{\text {K665D }}$ & this study \\
\hline pFLAGN1-4612 & pFLAGN1; NCU04612 & this study \\
\hline
\end{tabular}




\begin{tabular}{|c|c|c|}
\hline pFLAGN1-4612DAPM & pFLAGN1; NCU04612 212 D;T216D & this study \\
\hline pHAN1 & $\begin{array}{l}\text { Fungal expression vector for } \mathrm{N} \text {-terminal } \\
\mathrm{HA} \text { fusion proteins under control of } \mathrm{P}_{\mathrm{ccg}-} \\
\text { 1; target to his-3 locus }\end{array}$ & $\begin{array}{l}\text { (Kawabata \& Inoue, } \\
\text { 2007) }\end{array}$ \\
\hline pHAN1-8741 & pHAN1; NCU08741 & this study \\
\hline pHAN1-8741 ${ }^{\text {S409A }}$ & pHAN1; NCU08741 ${ }^{\text {S409A }}$ & this study \\
\hline pHAN1-8741 ${ }^{\text {S409E }}$ & pHAN1; NCU08741 ${ }^{\text {S409E }}$ & this study \\
\hline pHAN1-4202 & pHAN1; NCU04202 & this study \\
\hline pHAN1-4202 $2^{\mathrm{C} 116 \mathrm{Y}}$ & pHAN1; NCU04202 $2^{\mathrm{C116Y}}$ & this study \\
\hline pHAN1-pm-3314 & pHAN1; pm-NCU03314 & this study \\
\hline pCCG::N-3xMyc & $\begin{array}{l}\text { Fungal expression vector for } \mathrm{N} \text {-terminal } \\
3 \mathrm{xmyc} \text { fusion proteins under control of } \\
\mathrm{P}_{\mathrm{ccg}-1} \text {; target to his- } 3 \text { locus }\end{array}$ & (Honda \& Selker, 2009) \\
\hline pCCG::N-3xMyc-3576 & pCCG::N-3xMyc, NCU03576 & this study \\
\hline pMF272ATGtoATC & $\begin{array}{l}\text { Fungal expression vector for C-terminal } \\
\text { GFP fusion proteins under control of } \\
\text { P }_{\text {ccg-1 }} \text {; target to his-3 locus; start codon } \\
\text { ATG was mutated to ATC }\end{array}$ & $\begin{array}{l}\text { (Freitag et al., 2004), } \\
\text { vector was mutated in } \\
\text { this study }\end{array}$ \\
\hline $\begin{array}{l}\text { pMF272ATGtoATC- } \\
0772\end{array}$ & pMF272ATGtoATC; NCU00772 (gck-1) & this study \\
\hline $\begin{array}{l}\text { pMF272ATGtoATC- } \\
7296\end{array}$ & pMF272ATGtoATC; NCU07296 (cot-1) & this study \\
\hline $\begin{array}{l}\text { pMF272ATGtoATC- } \\
8741\end{array}$ & pMF272ATGtoATC; NCU08741 (ham-3) & this study \\
\hline $\begin{array}{l}\text { pMF272ATGtoATC- } \\
7674\end{array}$ & pMF272ATGtoATC; NCU07674 (mob-3) & this study \\
\hline $\begin{array}{l}\text { pMF272ATGtoATC- } \\
4202\end{array}$ & pMF272ATGtoATC; NCU04202 (ndk-1) & this study \\
\hline $\begin{array}{l}\text { pMF272ATGtoATC- } \\
3576\end{array}$ & pMF272ATGtoATC; NCU03576 (hym-1) & this study \\
\hline $\begin{array}{l}\text { pMF272ATGtoATC- } \\
11376\end{array}$ & pMF272ATGtoATC; NCU11376 (mak-1) & this study \\
\hline $\begin{array}{l}\text { pMF272ATGtoATC- } \\
\text { pm-7296 }\end{array}$ & $\begin{array}{l}\text { pMF272ATGtoATC; pm-NCU07296 (cot } \\
\text { 1) }\end{array}$ & this study \\
\hline $\begin{array}{l}\text { pMF272ATGtoATC- } \\
\text { pm-7296 }\end{array}$ & $\begin{array}{l}\text { pMF272ATGtoATC; pm-NCU07296 (cot } \\
\text { 1(T589A)) }\end{array}$ & this study \\
\hline pGFP::hph::IoxP & $\begin{array}{l}\text { Fungal expression vector for C-terminal } \\
\text { GFP fusion proteins under control of } \\
\text { the endogenous promotor }\end{array}$ & (Honda \& Selker, 2009) \\
\hline pGFP::hph::loxP-3576 & pGFP::hph::loxP; NCU03576 & this study \\
\hline pGFP::hph::loxP-7296 & pGFP::hph::loxP; NCU07296 & this study \\
\hline
\end{tabular}




\subsubsection{Plasmids for yeast two-hybrid analysis}

$N$. crassa cDNA for plasmids used in a yeast two-hybrid study was amplified by PCR from a cDNA library (www.FGSC.net). Proteins in this study were expressed as $\mathrm{N}$-terminal fusion constructs with SV40 NLS (nuclear localization signal)-GAL4 activation domain from pGADT7 or with GAL4 DNA binding domain from pGBKT7 (both from Clontech).

Full length MAPK (NCU02393), MAPKK (NCU04612) and MAPKKK (NCU06182) CDNAs were amplified using primers AD2393-EcoRI5 and AD2393-BamHI3; AD4612-EcoRI5 and AD4612BamHI3; AD6182-EcoRI5 and AD6182-BamHI3 and inserted into pGADT7 or pGBKT7. Expression plasmids used in the yeast two-hybrid assay encoded for the regions NCU00772 and NCU04202 were generated by PCR with Phusion ${ }^{\circledR}$ DNA polymerase (Finnzymes AG, Espoo, FIN) using the following primers AD0772-Ndel5 and AD0772-EcoRI3; AD4202-Ndel5 and AD4202-EcoRI3.

The pGADT7-Pro11N, pGADT7-Pro11C and pGADT7-Mob3 were constructed by Yasmine Bernhards. cDNA encoding regions were amplified from a Sordaria macrospora cDNA library and ligated into the pGADT7 vector.

\subsubsection{Constructs for overexpression of epitope-tagged fusion proteins for co- immunoprecipitation experiments}

To generate a vector for expression of 3xmyc-tagged HYM1 the corresponding coding region was amplified from genomic DNA using the primers ADMYC3576-Sgsl5 and ADMYC3576Spel3. The PCR fragment was cleaved and ligated into pCCG::N-3xMyc. The plasmid was linearized with Sspl for electroporation into N. crassa (see section 4.4.6).

$\mathrm{N}$-terminally HA-tagged versions of ham-3, gck-1 and ndk-1 were performed by using PCR, genomic wild type DNA and the respective primer pairs ADHA8741-Smal5 and ADHA8741EcoRI3; ADHA0772-BamHI5 and ADHA0772-EcoRI3; ADHA4202-BamHI5 and ADHA4202Pacl3 for amplification. The expression level of the HA-tagged fusion proteins after electroporation into the fungus were checked by Western blotting and immunodetection with anti-HA antibody (see section 4.5).

To create constructs of N-terminally 3xFLAG-tagged NCU07674, NCU04202, NCU06182 and NCU04612 fusion proteins, the corresponding coding sequences were amplified from genomic DNA using primers ADFLAG7674-Smal5 and ADFLAG7674-EcoRI3; ADFLAG4202BamHI5 and ADFLAG4202-Pacl3; ADFLAG6182-BamHI5 and ADFLAG6182-EcoRI3; ADFLAG4612-BamHI5 and ADFLAG4612-Pacl3. The DNA fragments were cleaved with the respective restriction enzymes and ligated into the pFLAGN1. The plasmids were linearized for electroporation into N. crassa.

The $n d k-1(C 116 Y)$ construct is presumed to represent an altered gene product that acts antagonistically to the wild type allele (Izumiya \& Yamamoto, 1995, Yamamoto et al., 1995). For this purpose a site-directed mutagenesis PCR was performed with the primer pair AD4202DN-MutP5 and AD4202DN-MutP3 using the plasmid pHA-4202 as template. After subcloning into pJet1.2 blunt vector, sequencing, digestion with restriction enzymes and 
insertion into the pHAN1 end vector, the plasmid was linearized and electroporated into the fungus.

\subsubsection{GFP plasmids for localization studies}

Constructs for GFP (green fluorescent protein)-tagged fusion proteins were generated by amplification of the ORFs as annotated by the $N$. crassa database (http://www.broad.mit.edu). Different restriction sites were added to the ends of the primers for introducing the DNA fragments into the corresponding PMF272ATGtoATC vector. For cot-1 the primer pair ADGFP7296-Xbal5 and ADGFP7296-BamHI3, for hym-1 the primer pair ADGFP3576-Xbal5 and ADGFP3576-BamHI3, for ham-3 the primer pair ADGFP8741Spel5 and ADGFP8741-Smal3, for gck-1 the primer pair ADGFP0772-Xbal5 and ADGFP0772BamHI3, for mob-3 the primer pair ADGFP7674-Xbal5 and ADGFP7674-Smal3, for $n d k-1$ the primer pair ADGFP4202-Xbal5 and ADGFP4202-Smal3, at least for mak-1 the primer pair ADGFP11367-SgsI5 and ADGFP11376-Pacl3 were used.

To generate C-terminal GFP-tagged hym-1 and cot-1 constructs under the endogenous promoter, the ORF of both were amplified by PCR using wild type DNA and the primer pairs DJ_Cot_Start_Xhol5 and DJ_Cot_Stopp_Xhol3; ADendoGFP3576-Xhol5 and ADendoGFP3576-Xhol3. After subcloning into the pJet1.2 blunt vector, sequencing and digestion with the respective restriction enzyme Xhol, the PCR fragments were introduced into the pGFP::hph::loxP vector. $1 \mathrm{~kb}$ fragment of the respective $3^{\prime} U T R$ was also amplified by PCR using the primer pairs DJ_Cot_3UTR_Sacl5 and DJ_Cot_3UTR_Sacl3 for cot-1; ADendoGFP3576-BamHI-3UTR5 and ADendoGFP3576-BamHI-3UTR3 for hym-1 to ensure homologous recombination at the endogenous locus in the fungus

\subsubsection{Dominant-active constructs of MAPKKK NRC1 and MAPKK MEK2 and a kinase-dead MAPKKK NRC1}

For generating dominant-active (DA) versions of the MAPKKK NRC1 (NCU06182) and the MAPKK (NC04612) site-directed mutagenesis PCRs with the primer pairs AD6182DAPMMutP5 and AD6182DAPM-MutP3, AD6182DADel-MutP5 and AD6182DADel-MutP3, AD4612DA-MutP5 and AD4612DA-MutP3 and the plasmids pFLAGN1-6182 either pFLAGN14612 as templates were performed according to manufacturer's manuals. The resulting plasmids pFLAGN1-6182DAPM, pFLAGN1-6182DADel and pFLAGN1-4612DAPM were linearized with Sspl, electroporated into $N$. crassa and MAK2 activity was determined by SDS-PAGE, Western blotting and immunodetection with a polyclonal anti-phospho-p44/42 MAPK (Thr202/Tyr204) antibody (see section 4.5.5).

To generate a kinase-dead construct of $n r c-1$ a site-directed mutagenesis PCR with the primer pair AD6182KD-5f and AD6182KD-3r and the plasmid pFLAGN1-6182 was performed. The resulting plasmid was electroporated into the fungus and the expression level was checked by Western blotting. The loss of kinase activity was verified by a kinase assay (see section 4.5.5). 


\subsection{Strains}

N. crassa strains used in this work are listed in Table 5. Several strains were obtained from the Fungal Genetic Stock Center (FGSC) at the University of Missouri, USA. Single deletion strains used in this study had been generated within the framework of the Neurospora genome project hosted at Dartmouth Medical School, Great Britain, following the procedure described in (Dunlap et al., 2007). Detailed descriptions are available at http://dartmouth.edu/ neurosporagenome/protocols.html. The full-length open reading frames were disrupted and replaced by a hygromycin resistance cassette and all strains were verified by Southern hybridization.

In this study the majority of $N$. crassa strains were produced by transformation applying plasmids targeted to the his-3 locus. These constructs contain the expression cassette flanked by the regions for homologous recombination to the his-3 locus and restoration of a functional his-3 allele (Margolin et al., 1997, Aramayo \& Metzenberg, 1996). Transformants expressing the desired protein were selected on minimal medium.

The expression levels of all fusion proteins were routinely checked by Western blotting (see 4.5.2) and immunodetection with the respective antibodies.

Strains for expression of GFP-tagged fusion proteins were generated by transforming the strain his-3 with the expression vectors targeted to the his-3 locus. Histidine-prototrophic transformants were selected on minimal medium. To test the functionality of the expressed fusion protein, the resulting strain was crossed with the respective hygromycin-resistant deletion mutant. The suppression of the phenotypic defect and the growth on hygromycin containing medium was used as evidence for fully functionality.

N. crassa strains expressing HA-, myc- and FLAG-tagged fusion proteins for coimmunoprecipitation studies were generated by transforming auxotrophic strains trp-3; his3 or nic-3; his-3. Histidine-prototrophic transformants were selected and the expression level of the fusion proteins was checked. The resulting strains still need medium supplemented with tryptophane or nicotinamide to grow. For co-immunoprecipitation studies, combinations of these strains were fused to generate prototrophic heterokaryotic strains expressing both fusion proteins. For this purpose, conidia of the respective strains (one Nic', second $\operatorname{Trp}^{-}$) were fused on VMM (Kawabata \& Inoue, 2007).

The strain mus52::bar his-3 was used to transform GFP-tagged fusion proteins at the endogenous locus by homologous recombination. Transformants were selected by their ability to grow on hygromycin and the expression level was checked by Western blotting. To remove the mus52 mutation the resulting strains were crossed with wild type or his-3 strain, verification by Southern analysis is required.

Table 5: $\boldsymbol{N}$. crassa strains used in this study. Genetic features are marked as (EC) for ectopical integration.

\begin{tabular}{l|l|l}
\hline \multicolumn{1}{c|}{ Strain } & \multicolumn{1}{c}{ Genotype or description } & \multicolumn{1}{c}{ Reference } \\
\hline wild type $A$ & 74-OR23-1V A & FGSC\#987 \\
\hline wild type $a$ & ORS-SL6 $a$ & FGSC\#4200 \\
\hline his-3 $A$ & his-3- $A$ & FGSC\#6103 \\
\hline \hline
\end{tabular}




\begin{tabular}{|c|c|c|}
\hline his-3a & his-3a & FGSC \#718 \\
\hline mus52::bar his-3 & $\Delta$ mus52::bar ${ }^{R}$ his-3- A & FGSC\#9720 \\
\hline trp-1, his-3 & $\operatorname{trp}-1^{-}$, his-3- & $\begin{array}{l}\text { S. Seiler (FGSC \#6103 x FGSC } \\
\# 4050)\end{array}$ \\
\hline nic-3, his-3 & nic-3, his-3- & $\begin{array}{l}\text { S. Seiler (FGSC \#6103 x FGSC } \\
\# 4082 \text { ) }\end{array}$ \\
\hline myc-cot-1;his-3 & myc-cot-1(183-4) his-3 - & (Maerz et al., 2009) \\
\hline myc-cot-1 & myc-cot-1(183-4) & (Ziv et al., 2009) \\
\hline myc-cot-1(T589E) & myc-cot-1(T589E) & (Ziv et al., 2009) \\
\hline $3 x F L A G-4202 t r p$ & $\begin{array}{l}\text { trp-1 } 1^{-} \text {is }-3^{+}:: \text {Pccg-1-3xflag- } \\
\text { NCU04202 }\end{array}$ & this study \\
\hline 3xFLAG-4202nic & $\begin{array}{l}\text { nic-3 his- } 3^{+}:: \text {Pccg-1-3xflag- } \\
\text { NCU04202 }\end{array}$ & this study \\
\hline $3 \times F L A G-7674 t r p$ & $\begin{array}{l}\text { trp-1'his-3 }{ }^{+}:: \text {Pccg-1-3xflag- } \\
\text { NCU07674 }\end{array}$ & this study \\
\hline $3 \times H A-0772 n i c$ & $\begin{array}{l}\text { nic-3 } 3^{-} \text {is }-3^{+}:: \text {Pccg-1-3xHA- } \\
\text { NCU00772 }\end{array}$ & this study \\
\hline $3 \times H A-0772 t r p$ & $\begin{array}{l}\operatorname{trp}-1^{-} \text {his- } 3^{+}:: \text {Pccg-1-3xHA- } \\
\text { NCU00772 }\end{array}$ & this study \\
\hline $3 \times H A-2393 t r p$ & $\begin{array}{l}\text { nic-3 } 3^{-} \text {is }-3^{+}:: \text {Pccg-1-3xHA- } \\
\text { NCU02393 }^{+}\end{array}$ & this study \\
\hline 3xHA-2393nic & $\begin{array}{l}\text { trp-1 his-3 } 3^{+}: \text {Pccg-1-3xHA- } \\
\text { NCU02393 }^{+}\end{array}$ & this study \\
\hline $3 x H A-4202 n i c$ & $\begin{array}{l}\text { nic-3-his-3 }{ }^{+}: \text {Pccg-1-3xHA- } \\
\text { NCU04202 }\end{array}$ & this study \\
\hline $3 \times H A-4202 t r p$ & $\begin{array}{l}\text { trp-1 his-3 } 3^{+}: \text {Pccg-1-3xHA- } \\
\text { NCU04202 }\end{array}$ & this study \\
\hline $3 \times H A-4202$ & 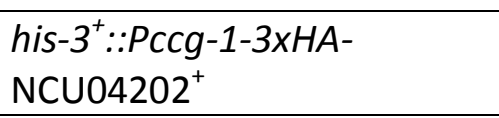 & this study \\
\hline $3 \times H A-8741$ & $\begin{array}{l}\text { his }-3^{+}:: P c c g-1-3 \times H A- \\
\text { NCU08741 }^{+}\end{array}$ & this study \\
\hline $3 \times H A-8741^{S 408 A}$ & $\begin{array}{l}\text { his }-3^{+}:: P c c g-1-3 \times H A- \\
\text { NCU08741 }\end{array}$ & this study \\
\hline $3 \times H A-8741^{S 408 E}$ & $\begin{array}{l}\text { his- } 3^{+}:: P c c g-1-3 \times H A- \\
\text { NCU08741 }\end{array}$ & this study \\
\hline $3 \times H A-8741 n i c$ & $\begin{array}{l}\text { nic-3 } 3^{-} \text {is }-3^{+}:: \text {Pccg-1-3xHA- } \\
\text { NCU08741 }^{+}\end{array}$ & this study \\
\hline $3 \times H A-8741$ trp & $\begin{array}{l}\text { trp-1 his- } 3^{+}:: \text {Pccg-1-3xHA- } \\
\text { NCU08741 }^{+}\end{array}$ & this study \\
\hline $3 x H A-0772 ; m y c-c o t-1$ & $\begin{array}{l}\text { myc-cot-1(183-4)his- } \\
3^{+}:: P c c g-1-3 x H A-N C U 00772^{+}\end{array}$ & this study \\
\hline $3 \times H A-8741 ; m y c-c o t-1$ & $\begin{array}{l}\text { myc-cot-1(183-4)his- } \\
3^{+}:: P c c g-1-3 x H A-N C U 08741^{+}\end{array}$ & this study \\
\hline 8741-sgfp (compl.) & $\begin{array}{l}\Delta \mathrm{NCU} 08741:: h p h^{R} \text { his- } \\
3^{+}:: P c c g-1-N C U 8741^{+} \text {-sgfp }\end{array}$ & this study \\
\hline 0772-sgfp (compl.) & 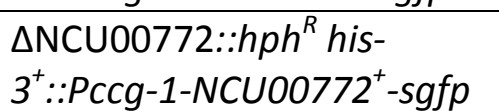 & this study \\
\hline
\end{tabular}




\begin{tabular}{|c|c|c|}
\hline 7674-sgfp (compl.) & $\begin{array}{l}\text { NCU07674::hph }{ }^{R} \text { his- } \\
3^{+}:: P c c g-1-N C \cup 07674^{+} \text {-sgfp }\end{array}$ & this study \\
\hline sgfp-11376 (compl.) & $\begin{array}{l}\Delta N C U 11376:: h_{p h}^{R} h^{\prime} s^{-} \\
3^{+}:: P c c g-1-s g f p-N C U 11376^{+}\end{array}$ & this study \\
\hline 4202-sgfp (compl.) & $\begin{array}{l}\Delta N C U 04202:: h p h^{R} \text { his- } \\
3^{+}:: P c c g-1-N C U 4202^{+} \text {-sgfp }\end{array}$ & this study \\
\hline 7296-sgfp (compl.) & 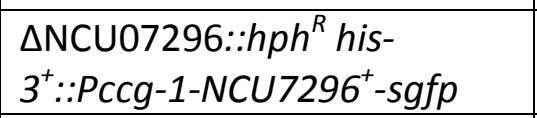 & this study \\
\hline 3576-sgfp & $\begin{array}{l}\text { his-3 } 3^{+}:: P c c g-1-N C U 03576^{+}- \\
\text {sgfp }\end{array}$ & this study \\
\hline 3576-sgfp (endo.) & $\begin{array}{l}\Delta \text { mus52::bar }{ }^{R} \text { his-3 Phym-1- } \\
\text { sgfp-hym-1 } \text { hyg }^{R} \text { (EC) }\end{array}$ & this study \\
\hline 7296-sgfp (endo.) & $\begin{array}{l}\Delta \text { mus52::bar }{ }^{R} \text { his-3 Pcot-1- } \\
\text { sgfp-cot-1 }{ }^{+} h y g^{R}(E C)\end{array}$ & this study \\
\hline 7296-sgfg & his-3 $3^{+}:: P c c g-1-N C U 7296^{+}-$sgfp & this study \\
\hline$p m-7296^{T 589 A}-G F P$ & $\begin{array}{l}\text { his- } 3^{+}:: \text {Pccg-1-pm-NCU7296 } \\
\text { T589A }^{+}-\text {sgfp }\end{array}$ & this study \\
\hline$p m-7296-G F P$ & $\begin{array}{l}\text { his- } 3^{+}:: P c c g-1-p m-N C U 7296^{+}- \\
\text {sgfp }\end{array}$ & this study \\
\hline $3 x F L A G-4612$ & $\begin{array}{l}\text { his- } 3^{+}:: \text {Pccg-1-3xflag- } \\
\text { NCU04612 }\end{array}$ & this study \\
\hline $3 x F L A G-4612^{\text {S212D;T216D }}$ & $\begin{array}{l}\text { his-3 }{ }^{+}:: P c c g-1-3 \times f l a g- \\
\text { NCU04612 }\end{array}$ & this study \\
\hline $3 x F L A G-6182$ & $\begin{array}{l}\text { his- } 3^{+}:: P c c g-1-3 x f l a g- \\
\text { NCU06182 }^{+}\end{array}$ & this study \\
\hline $3 \times F L A G-6182^{\mathrm{P} 448 \mathrm{~S}}$ & $\begin{array}{l}\text { his- } 3^{+}:: \text {Pccg-1-3xflag- } \\
\text { NCU06182 }\end{array}$ & this study \\
\hline $3 \times$ FLAG-6182 deletion aa41-590 & $\begin{array}{l}\text { his-3 }{ }^{+}:: P c c g-1-3 x f l a g- \\
\text { NCU06182 deletion aa41-590+ }\end{array}$ & this study \\
\hline $3 \times F L A G-6182^{\mathrm{K} 665 \mathrm{D}}$ & $\begin{array}{l}\text { his- } 3^{+}:: P c c g-1-3 x f l a g- \\
\text { NCU06182 } \\
\text { K665D+ }\end{array}$ & this study \\
\hline$p m-H A-m o b-2 a$ & pm-HA-mob-2a::his-3 & this study \\
\hline$p m-H A-m o b-2 a ; \Delta p o d-6$ & $\begin{array}{l}\text { pm-HA-mob-2a::his-3; } \\
\Delta p o d-6:: h p h^{R}\end{array}$ & this study \\
\hline$p m-m y c-c o t-1$ & pm-myc-cot1::his-3 & this study \\
\hline pm-myc-cot-1(T589A) & pm-myc-cot1(T589A)::his-3 & this study \\
\hline pm-myc-cot-1(T589E) & pm-myc-cot1(T589E)::his-3 & this study \\
\hline$\Delta p o d-6 ; p m-m y c-c o t-1$ & $\begin{array}{l}\text { pm-myc-cot1::his-3; } \\
\Delta \text { pod-6::hph }\end{array}$ & this study \\
\hline pm-myc-cot-1(T589A); $\Delta$ pod-6 & pm-myc-cot1(T589A)::his-3; & this study \\
\hline
\end{tabular}




\begin{tabular}{|c|c|c|}
\hline & spod-6::hph ${ }^{R}$ & \\
\hline pm-myc-cot-1(T589E); $\Delta p o d-6$ & $\begin{array}{l}\text { pm-myc-cot1::his-3(T589A); } \\
\text { spod-6::hph }\end{array}$ & this study \\
\hline HA-pod-6;myc-cot-1 & myc-Pcot-1-cot-1,HA-pod6 & this study \\
\hline myc-cot-1-HA-pod6; $\Delta$ hym-1 & $\begin{array}{l}\text { myc-Pcot-1-cot-1,HA-pod6; } \\
\text { Shym1::hph }\end{array}$ & this study \\
\hline$\Delta p o d-6$ & $\Delta p o d-6:: n a t R$ & S. Seiler \\
\hline$\Delta h y m-1 a$ & $\Delta h y m-1:: h p h^{R} a$ & FGSC\#13043 \\
\hline$\Delta m o b-3 a$ & $\Delta m o b-3:: h p h^{R} a$ & FGSC\#12362 \\
\hline$\Delta h a m-3 A$ & $\Delta h a m-3:: h p h^{R} A$ & FGSC\#11299 \\
\hline$\Delta h a m-3 a$ & $\Delta h a m-3:: h p h^{R} a$ & FGSC\#11300 \\
\hline$\Delta g c k-1 A$ & $\Delta g c k-1:: h p h^{R} A$ & S. Seiler \\
\hline$\Delta g c k-1 a$ & $\Delta g c k-1:: h p h^{R} a$ & FGSC \#11478 \\
\hline$\Delta m a k-1 a$ & $\Delta m a k-1:: h p h^{R} a$ & FGSC 11321 \\
\hline$\Delta n r c-1 a$ & $\Delta n r c-1:: h p h^{R} a$ & FGSC \#18162 \\
\hline$\Delta m e k-2 a$ & $\Delta m e k-2:: h p h^{R} a$ & FGSC \#11481 \\
\hline$\Delta \cot -1 a$ (het) & $\begin{array}{l}\Delta \cot -1:: \text { hph }^{R}+\cot ^{+} 1^{+} \\
\Delta \text { mus51::bar }{ }^{R} a\end{array}$ & FGSC \#14525 \\
\hline$\Delta n d k-1 A$ & $\Delta n d k-1:: h p h^{R} A$ & FGSC \#14552 \\
\hline$\Delta n d k-1 a$ & $\Delta n d k-1:: h p h^{R} a$ & S. Seiler \\
\hline
\end{tabular}

For amplification of plasmids the Escherichia coli strain DH5 $\alpha$ [F-, $\Phi 80 \mathrm{~d} \Delta($ lacZ)M15-1, $\triangle($ lacZYA-argF)U169, recA1, endA1, hsdR17 ( $\mathrm{rK}-, \mathrm{mK}+$ ), supE44, $\lambda-$, thi1, gyrA96, relA1] (Woodcock, 1989) was used.

For yeast two-hybrid analyses the Saccharomyces strain AH109 [MATa, trp1-901, leu2-3, 112, ura3-52, his3-200, gal4D, gal804, LYS2::GAL1 UAS-GAL1 TATA $-H I S 3$, GAL2 UAS-GAL2 TATA $-A D E 2$,

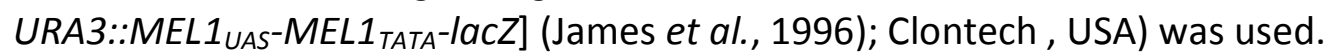

\subsection{General molecular biological methods}

Standard molecular methods were performed as described in (Sambrook \& and Russell, 2001, Ausubel et al., 2002) and partially modified or adapted from manufacturer's manuals.

\subsubsection{Genomic DNA preparation and Southern hybridization}

For isolation of genomic DNA from N. crassa, the methods of (Weiland, 1997, Borges et al., 1990) were used. Mycelium from liquid cultures were harvested and ground to a fine powder with mortar and pestle. The samples were homogenized in extraction buffer $(50 \mathrm{mM}$ Tris, $\mathrm{pH}$ 8.0, 50mM ethylenediaminetetraacetic acid (EDTA), 3\% sodium dodecyl sulfate (SDS), $1 \% \beta$-mercaptoethanol) and incubated for two hours at $65^{\circ} \mathrm{C}$. Subsequently, three extraction steps with Roti ${ }^{\circledR}$-Phenol (Carl Roth GmbH \& Co KG, Germany)/chloroform, isoamyl alcohol (25/24/1) and one with chloroform were performed. Now DNA was precipitated with 
isopropanol ( 0.7 volumes added), redissolved in 1xTE buffer (10mM Tris, pH8.0, 1mM EDTA) and ribonucleic acid (RNA) was digested by adding $0.1 \mu \mathrm{g} / \mathrm{ml}$ RNaseA (Fermentas $\mathrm{GmbH}$, Germany) and incubated for $10 \mathrm{~min}$ at $65^{\circ} \mathrm{C}$. Thereupon DNA was precipitated again by addition of $1 / 10$ volume of $3 \mathrm{M}$ sodium acetate and 2 volumes of $100 \%$ ethanol. Nucleic acid was washed once with $70 \%$ ethanol, air-dried, redissolved in $\mathrm{H}_{2} \mathrm{O}$ and stored at $4^{\circ} \mathrm{C}$.

In some cases for genomic DNA preparation the Wizard ${ }^{\circledR}$ Genomic DNA Purification Kit (Promega GmbH, Madison, Germany) according to the Plant Tissue Protocol was used.

For Southern blot analysis Gene ImagesTM Random-Prime DNA labelling kit and the Gene ImagesTM CDP-StarTM Detection Kit (GE Healthcare Life Sciences, Munich, Germany) for non-radioactive labelling and detection were used.

\subsubsection{Polymerase chain reaction (PCR)}

Phusion ${ }^{\circledR}$ High Fidelity polymerase (Finnzymes AG, Espoo, FIN) was used for amplification of DNA by polymerase chain reaction in accordance with standard protocols (Ausubel et al., 2002) or with manufacturer's manuals. Analytical PCRs were performed with Taq DNA polymerase. Oligonucleotides used as PCR primers were ordered from Eurofins MWG Operon (Ebersberg, Germany) or Invitrogen $\mathrm{GmbH}$ (Karlsruhe, Germany). Plasmids, genomic DNA or CDNA were used as templates for the reactions depending on the purpose. In case of colony PCRs (Zon et al., 1989) which were performed to identify positive $E$. coli transformants, E. coli cells of a single colony were directly used as templates for the reaction.

A standard PCR reaction consisted of an initial template denaturation $\left(2 \mathrm{~min}, 94^{\circ} \mathrm{C}\right.$ ) followed by 35 cycles each cycle consisting of $30 \mathrm{~s}$ denaturation at $94^{\circ} \mathrm{C}$, primer annealing $(30 \mathrm{~s}, 50$ $60^{\circ} \mathrm{C}$ depending on the primers used), product elongation step at $72^{\circ} \mathrm{C}(1 \mathrm{~min} / 1 \mathrm{~kb}$ template length), and a final elongation step of $3 \mathrm{~min}$ at $72^{\circ} \mathrm{C}$.

Site-directed mutagenesis was performed with the Phusion ${ }^{\circledR}$ High Fidelity polymerase (Finnzymes AG, Espoo, FIN) according to the QuickChangeR Site-Directed Mutagenesis Kit protocol (Stratagen, La Jolla, USA). The amplified PCR products were directly digested with $D p n l$ to separate the new mutated non-methylated target plasmid from the non-mutated methylated input plasmid. The digested DNA was subsequently transformed into $E$. coli.

\subsubsection{DNA agarose gel electrophoresis and isolation}

DNA fragments and plasmids were separated by horizontal agarose gel electrophoresis using the Mini Sub-Cell System (Bio-Rad Laboratories GmbH, Germany). Gene Ruler ${ }^{T M} 1 \mathrm{~kb}$ DNA ladder (Fermentas $\mathrm{GmbH}$, Germany) was used as a DNA molecular weight marker und the DNA was stained with ethidiumbromide. The stained DNA was visualized by using the Molecular Imager Gel Doc XR System (Bio-Rad Laboratories GmbH, Germany).

DNA gel extraction was performed using the peqGOLD Gel Extraction Kit (PeqLab GmbH, Erlangen, Germany) and plasmid DNA from E. coli was prepared using peqGOLD Plasmid Miniprep Kit II (PeqLab GmbH, Erlangen, Germany) according to manufacturer's manuals. 


\subsubsection{Enzymatic digestion of DNA and modification}

For endonucleolytic digestion of DNA, restriction enzymes and buffers were ordered from Fermentas GmbH (Germany) and New England Biolabs GmbH (USA) and used in accordance to product manuals.

DNA termini of cloning vectors were dephosphorylated using Shrimp Alkaline Phosphatase (Fermentas $\mathrm{GmbH}$, Germany) to prevent vector recircularization in accordance to manufacturer's recommendations.

\subsubsection{Ligation}

Ligation reactions were performed in $20 \mu$ reaction volumes using T4 DNA ligase (Fermentas $\mathrm{GmbH}$, Germany) in a 2 fold concentrated quick ligation buffer (50mM HEPES pH 7.6, 10mM $\mathrm{MgCl}_{2}, 2 \mathrm{mM}$ DTT, 2mM ATP and 7\% (v/v) PEG4000) for $30 \mathrm{~min}$ at room temperature (RT).

\subsubsection{DNA Transformation}

DNA transformation was performed as described in Inoue et al., 1990 by using chemically competent $E$. coli cells.

Plasmids were transformed by electroporation of conidia as mentioned in Margolin et al., 1997 with minor modifications. The $N$. crassa conidia were harvested after 9-11 days and the electroporation was performed in cuvettes obtained from PEQLAB Biotechnologie GmbH (Germany) with these settings (voltage $1.5 \mathrm{kV}$; capacitance: $50 \mu \mathrm{F}$; resistance: $200 \Omega$ ). The conidia were resuspended in $1 \mathrm{M}$ sorbitol and plated on minimal medium. For selection of transformants with dominant markers, the conidia were resuspended in VMM, incubated for 3 hours at room temperature and were plated on selective medium.

\subsubsection{Sequence analysis}

DNA was sequenced by Göttingen Genomics Laboratory at the Institute of Microbiology and Genetics, University of Göttingen (G2L, Göttingen, Germany). Sequences were analysed using 4Peaks (version 1.7.2; Mekentosj B.V., The Netherland) and Lasergene (DNASTAR, Inc., Madison, USA) software. Alignments of sequences were performed with BLAST searches at NCBI (http://www.ncbi.nlm.nih.gov/) or MultAlin

(http://bioinfo.genopoletoulouse.prd.fr/multalin/multalin.html).

\subsection{Biochemical and immunological techniques}

\subsubsection{Protein isolation of $N$. crassa}

$N$. crassa strains were grown in $50 \mathrm{ml}$ liquid minimal medium, harvested by filtration using a Büchner funnel and ground in liquid nitrogen. The pulverized mycelium was homogenized in protein extraction buffer $(50 \mathrm{mM}$ Tris $\mathrm{pH} 7.5,100 \mathrm{mM} \mathrm{KCl}, 10 \mathrm{mM} \mathrm{MgCl} 2,0.1 \% \mathrm{NP} 40$; freshly added $2 \mathrm{mM}$ benzamidine, $2 \mathrm{mM}$ DTT, $1 \mathrm{mM}$ Pefabloc SC). After a centrifugation step (16000g) at $4^{\circ} \mathrm{C}$ for $10 \mathrm{~min}$ the clear supernatant was mixed with $3 x$ Laemmli sample buffer $(10 \%$ 
glycerol, $5 \% \beta$-mercaptoethanol, $15 \%$ SDS, $12,5 \%$ upper-buffer (0,5 M Tris- $\mathrm{HCl}$ pH6.8), 0,75\% bromophenol blue, $3 \mathrm{M}$ urea; modified from Laemmli, 1970 and boiled at $98^{\circ} \mathrm{C}$ for $10 \mathrm{~min}$.

\subsubsection{Separation of proteins by SDS polyacrylamide gel electrophoresis (SDS-PAGE) and Western blotting}

Protein samples boiled in Laemmli buffer were loaded on a vertical discontinuous polyacrylamide gel electrophoresis (PAGE) (Davis, 1964, Ornstein, 1964) in the presence of sodium dodecyl sulfate (SDS) (Laemmli, 1970) by using the Mini-Protean ${ }^{\circledR} 3$ Cell System (BioRad Laboratories $\mathrm{GmbH}$, Germany). Electrophoretic separation was performed by a constant current of $15 \mathrm{~mA}$ per gel submerged in running buffer $(2.5 \mathrm{mM}$ Tris base, $19.2 \mathrm{mM}$ glycine and $0.1 \%$ SDS). Generally, the molecular weight marker PageRuler ${ }^{\mathrm{TM}}$ Prestained Protein Ladder (Fermentas $\mathrm{GmbH}$, Germany) was loaded onto the gels for molecular weight determination of proteins.

Proteins were visualized by staining with Coomassie Brilliant Blue $0.1 \%$ Coomassie Brilliant Blue, $40 \%$ methanol, $10 \%$ acetic acid; (Merril, 1990) or alternatively by Western blotting.

For this purpose, proteins were transferred electrophoretically from polyacrylamide gels to Protran ${ }^{\circledR}$ nitrocellulose membrane (Whatman $\mathrm{GmbH}$, Germany) performed in Mini TransBlot $^{\circledast}$ Cells (Bio-Rad Laboratories $\mathrm{GmbH}$, Germany). After electroblotting for 1 hour at 100V, the nitrocellulose membrane was stained reversible with Ponceau S $(0.1 \%$ Ponceau $S$ in $5 \%$ acetic acid) (Salinovich \& Montelaro, 1986). Their immunological detection (Western blotting) was based on the method described by Towbin et al., 1979. To prepare the membrane for incubation with antibodies an initial blocking step with 5\% Sucofin milk powder (TSI GmbH \& Co. KG, Germany) in PBS solution (10mM sodium phosphate, $150 \mathrm{mM}$ $\mathrm{NaCl}, \mathrm{pH}$ 7.4) was performed. Unbound antibodies were washed off with PBS. Mouse monoclonal anti -c-myc antibody 9E10 (Santa Cruz Biotechnology, Heidelberg, Germany), anti-GFP (B-2) (Santa Cruz Biotechnology, Heidelberg, Germany), anti-HA (clone HA-7) or anti-FLAG ${ }^{\circledR}$ M2 (both Sigma-Aldrich, Taufkirchen, Germany) or polyclonal anti-phosphop44/42 MAPK (Thr202/Tyr204) (Cell Signaling Technology, Inc., USA) were used as primary antibodies and were detected by peroxidase-coupled goat anti-mouse IgG or goat anti-rabbit IgG antibody (Invitrogen $\mathrm{GmbH}$, Karlsruhe, Germany). Detection was performed using Immobilon ${ }^{\text {TM }}$ Chemiluminescent Western HRP Substrate (Millipore, USA) in combination with Amersham $^{\mathrm{TM}}$ Hyperfilm $^{\mathrm{TM}}$ ECL (GE Healthcare Europe GmbH, Germany).

\subsection{3 (Co-) Immunoprecipitation analysis}

For immunoprecipitation the $N$. crassa strains were grown in liquid minimal medium. The mycelium was harvested by filtration and ground in liquid nitrogen. All buffers used for this experiment contained the following additives: $25 \mathrm{mM} \beta$-glycerophosphate, $10 \mathrm{ng} / \mu \mathrm{l}$ leupeptine, $10 \mathrm{ng} / \mu \mathrm{l}$ aprotinine, $2 \mathrm{ng} / \mu \mathrm{l}$ Pepstatin A, $2 \mathrm{mM}$ DTT, $1 \mathrm{mM}$ Pefabloc SC, $2 \mathrm{mM}$ benzamidine, $5 \mathrm{mM} \mathrm{NaF}$ and $1 \mathrm{mM} \mathrm{Na}_{3} \mathrm{VO}_{4}$. The pulverized mycelium was homogenized in lysis buffer ( $50 \mathrm{mM}$ Tris pH7.5, $\left.100 \mathrm{mM} \mathrm{KCl}, 10 \mathrm{mM} \mathrm{MgCl}_{2}, 0.1 \% \mathrm{NP}-40\right)$ and centrifuged in two steps at $4^{\circ} \mathrm{C}(25 \mathrm{~min}$ at $4500 \mathrm{~g}$ followed by a second centrifugation step for 1 hour at $16000 \mathrm{~g})$. The clear lysate was then incubated on a rotation device for two hours with $4 \mu \mathrm{l} / \mathrm{ml}$ lysate monoclonal mouse anti-c-myc, $4 \mu \mathrm{l} / \mathrm{ml}$ lysate monoclonal mouse anti-GFP, $2 \mu \mathrm{l} / \mathrm{ml}$ 
lysate monoclonal mouse anti-HA or $2 \mu \mathrm{l} / \mathrm{ml}$ lysate monoclonal mouse anti-FLAG ${ }^{\circledR} \mathrm{M} 2$ antibody and with $5 \mathrm{mg} / \mathrm{ml}$ lysate Protein-A-Sepharose beads (GE Healthcare Life Sciences, USA) for an additional hour at $4^{\circ} \mathrm{C}$. Subsequently, the lysate-antibody-sepharose mixture was centrifuged $(1 \mathrm{~min}$ at $16000 \mathrm{~g}$ ) and washed twice with lysis buffer. Immunoprecipitated proteins were recovered by boiling the sepharose beads for $10 \mathrm{~min}$ at $98^{\circ} \mathrm{C}$ in $3 \mathrm{x}$ Laemmli buffer. The boiled samples were loaded on SDS-PAGE gel and stained with Coomassie Brilliant Blue or alternatively a Western blotting and immunodetection were performed (see 4.5.2).

\subsubsection{Analysis of MAK2 phosphorylation status}

Liquid $N$. crassa cultures were grown at room temperature. Oxidative stress was induced by addition of $8 \mathrm{mM} \mathrm{H}_{2} \mathrm{O}_{2}$ for $30 \mathrm{~min}$ (Merck KGaA, Germany) whereat half of the cultures were left untreated to determine basal level of phosphorylation. The mycelium was harvested gently by filtration using a Büchner funnel and was ground in liquid nitrogen. Protein extraction was performed as described in Jones et al., 2007 with modifications communicated by L. Bennett (Department of Biology, Texas A\&M University, USA). The pulverized mycelium was transferred to a screw-top tube with glass beads (diameter 0.25$0.5 \mathrm{~mm}$ ) and $1 \mathrm{ml}$ of $95 \%$ ethanol was added. The samples were mixed and incubated overnight at $-20^{\circ} \mathrm{C}$. After the inoculation step the samples were centrifuged for $30 \mathrm{~min}$ at $16000 \mathrm{~g}$ and $4^{\circ} \mathrm{C}$ and the supernatant was removed. Subsequently, the pellet was vacuumdried in a SpeedVac concentrator (Thermo Fisher Scientific, USA). In a next step, extraction buffer (100mM Tris pH7.0, 1\% SDS and freshly added 10ng/ $\mu$ l aprotinine, $2 \mathrm{ng} / \mu \mathrm{l}$ Pepstatin A, $2 \mathrm{mM}$ DTT, $1 \mathrm{mM}$ Pefabloc SC, $2 \mathrm{mM}$ benzamidine, $5 \mathrm{mM} \mathrm{NaF}$ and $1 \mathrm{mM} \mathrm{Na}_{3} \mathrm{VO}_{4}$ ) was added, the samples were mixed and incubated for $5 \mathrm{~min}$ at $80^{\circ} \mathrm{C}$. After a centrifugation step at RT for $5 \mathrm{~min}$ at $16000 \mathrm{~g}$ the supernatant was transferred to a new collection tube. The extraction step was repeated once and the supernatants were pooled. In a further centrifugation step $(15 \mathrm{~min}$ at $16000 \mathrm{~g})$ the lysates were cleared and the protein contents were measured spectrophotometrically using a Nanodrop spectrophotometer ND-1000 (analysis mode Protein A280, 340nm normalization on, PEQLAB Biotechnologie GmbH, Germany). Finally, the clear lysates were mixed with $3 x$ Laemmli buffer and were boiled for $10 \mathrm{~min}$ at $98^{\circ} \mathrm{C}$.

Around $75 \mu \mathrm{g}$ of total protein per lane were loaded onto SDS polyacrylamide gels followed by electrophoresis and Western blotting (see 4.5.2). After blocking the nitrocellulose membrane in $5 \%$ milk powder solved in PBS-Tween (PBS solution added with $0.05 \%$ Tween20), the membrane was washed three times with PBS-Tween. For immunodetection polyclonal anti-phospho-p44/42 MAPK (Thr202/Tyr204) antibody in 5\% bovine serum albumin/PBS-Tween was used as primary and goat anti-rabbit IgG antibody as secondary antibody.

The AIDA Image Analyzer (version 4.22; raytest Isotopenmessgeräte $\mathrm{GmbH}$, Germany) in transmission mode was used for quantification. The exposed films were scanned and saved as tiff-files for determination of MAK2 phosphorylation levels. The MAK2 protein bands of interest were measured, corrected by substraction of local background and normalized to the protein amount loaded. Basal and stress-induced MAK2 phosphorylation levels of mutant strains were related to levels of the wild type. Each experiment consisted of three to four individual cultures under non-stress and stress conditions. 


\subsubsection{Kinase assay}

Immunoprecipitation of the kinase followed the protocol described in section 4.5.3. After incubating the lysate-antibody compound with Protein-A-Sepharose the resulting mixture was centrifuged for $1 \mathrm{~min}$ at $16000 \mathrm{~g}$ and was washed once with lysis buffer. The sepharoses with the potential immunoprecipitated kinase and the respective immunoprecipitated substrate were pooled and further washing steps were accomplished (two times with lysis buffer containing $500 \mathrm{mM} \mathrm{NaCl}$ and further 2 times with kinase buffer (20mM Tris pH7.5, $10 \mathrm{mM} \mathrm{MgCL} 2,1 \mathrm{mM}$ DTT, $1 \mathrm{mM}$ benzamidine, $5 \mathrm{mM} \mathrm{NaF}$ and $\left.2 \mathrm{mM} \mathrm{Na}_{3} \mathrm{VO}_{4}\right)$ ). After the washing steps a mastermix for the radioactive kinase reaction was prepared in which $10 \mu \mathrm{Ci}$ of radioactive-labeled $\gamma$-ATP ${ }^{\mathrm{P} 32} / 100 \mu \mathrm{l}$ kinase buffer was used $(50 \mu \mathrm{l}$ kinase buffer with radioactive-labeled $\mathrm{Y}-\mathrm{ATP}^{\mathrm{P} 32}$ per single reaction) and incubated for $1 \mathrm{~h}$ at $37^{\circ} \mathrm{C}$. The samples were then centrifuged for $1 \mathrm{~min}$ at $16000 \mathrm{~g}$, the supernatant was discarded and the sepharose beads were boiled in $3 x$ Laemmli buffer for $10 \mathrm{~min}$ at $98^{\circ} \mathrm{C}$. Finally the extracts were loaded onto SDS gels and Western blotting with immunodetection or an autoradiograph was performed. For an autoradiograph the SDS polyacrylamide gels were dried and Amersham ${ }^{\mathrm{TM}}$ autoradiogram films (GE Healthcare Europe $\mathrm{GmbH}$, Germany) were used for visualization.

\subsection{Yeast two-hybrid studies}

For yeast two-hybrid analysis (Fields \& Song, 1989), the Matchmaker ${ }^{\mathrm{TM}}$ Two-Hybrid System 3 (Clontech, USA) was used according to manufacturer's manuals. Plasmids encoding proteins fused to the GAL4 activation domain (cDNA constructs inserted into pGADT7) or the DNAbinding domain (cDNA constructs inserted into pGBKT7) were cotransformed into the strain S. cerevisiae AH109 as described in protocols of Schiestl \& Gietz, 1989. Growing cotransformants were selected on SD medium lacking leucine and tryptophane by their restored ability to grow. Positive interactions of fusion proteins were shown by their ability to activate the reporter genes HIS3 and ADE2. For a yeast drop test, single colonies were collected, suspended in water and serial dilutions were plated on SD medium. To exclude autoactivation of the fusion proteins each plasmid was tested with the empty vector of the counterpart. General yeast two-hybrid competency of the DNA-binding domain fusion proteins was determined by their ability to interact with $\triangle N$-RanBPM (Tucker et al., 2009).

\subsection{Microscopy}

For stereomicroscopial documentation of fungal hyphae or colonies an Olympus SZX16 with an Olympus SDF PLAPO 1xPF objective and a Colorview III camera operated by the Cell ${ }^{\mathrm{D}}$ analysis Image Processing software (Olympus, Tokyo, Japan) was used.

Fluorescence microscopy of vegetative hyphal cells of $N$. crassa grown on thin VMM layers for 12-16 hours was performed as described in Hickey et al., 2002. Calcofluor White $(2 \mu \mathrm{g} / \mathrm{ml}$ dissolved in water; Sigma-Aldrich, Taufkirchen, Germany) was used for counterstaining of cell wall material and plasma membranes with Spitzenkörper were stained with FM4-64 $(1 \mu \mathrm{g} / \mathrm{ml}$ dissolved in water; Invitrogen $\mathrm{GmbH}$, Germany). Microscopy was performed with an inverted Axio Observer Z1 (Carl Zeiss AG, Oberkochen, Germany) microscope equipped with a QuantEM 512SC camera (Photometrics, Tucson, USA). For image acquisition the Slidebook 
5.0 software (Intelligent Imaging Innovations, $\mathrm{GmbH}$, Göttingen, Germany) was used and images were converted into TIFF files. 


\section{Results}

\subsection{Analysis of the COT1 kinase network in the fungus $N$. crassa}

\subsubsection{The COT1-MOB2 complex is important for polar growth}

NDR kinases are important for polar growth and differentiation in uni- and multicellular organisms. The NDR signaling network consists of the NDR kinase itself, kinase adaptor proteins termed MOBs (Bichsel et al., 2004, Hergovich et al., 2005) and an upstream Ste20 kinase (Hergovich et al., 2006a, Hergovich et al., 2006b, Kanai et al., 2005, Nelson et al., 2003, Stegert et al., 2005, Wei et al., 2007). It is known that the N-terminus, which is conserved in fungal and animal NDR kinases is important for the binding of MOB (Bichsel et al., 2004, He et al., 2005, Song et al., 2008). Next to this proposed MOB binding interface, most fungal NDR kinases contain an uncharacterized N-terminal extension.

To characterize the $\mathrm{N}$-terminusof the $N$. crassa NDR kinase COT1, constructs of two COT1 isoforms (amino acids 1-212 for the long isoform containing the N-terminus with the fungal specific N-terminal extension and aa 119-212 for the short isoform containing only the $\mathrm{N}$ terminus) were generated for yeast two-hybrid experiments. Interactions were detected between both COT1 fragments and MOB2A, indicating that region 119-212 of COT1 is sufficient for interaction. Growth curves of two-hybrid cultures under selective conditions confirmed a stronger interaction of MOB2A with the COT1 region 1-212 than with the region 119-212 (Maerz et al., 2009) (Figure 12B). Moreover, it was observed that the long fragment alone also interacted with MOB2B (Figure 12A).

In addition, COT1 dimerization was observed between both COT1 isoforms and the COT1 full length. Also both long fragments could dimerize, whereas the two short isoforms could not (Figure 12A). Thus, region $119-212$ is also sufficient for homo-dimerization, and the interaction is stabilized by amino acids 1-118. In summary, COT1 dimerization and COT1MOB2 binding occurred via the same $\mathrm{N}$-terminal domain. 
A

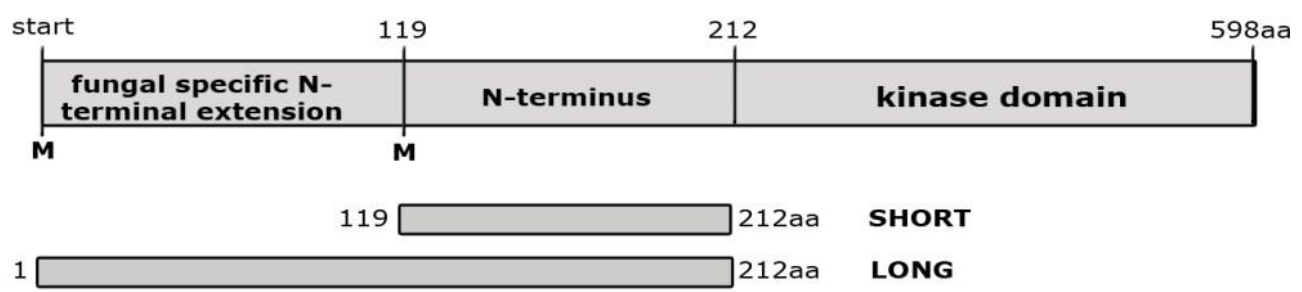

\begin{tabular}{|c|c|c|}
\hline $\begin{array}{c}\text { LONG } \\
+ \\
\text { LONG }\end{array}$ & $\begin{array}{c}\text { SHORT } \\
+ \\
\text { SHORT }\end{array}$ & $\begin{array}{c}\text { LONG } \\
+ \\
\text { COT1 }\end{array}$ \\
\hline $\begin{array}{c}\text { SHORT } \\
+ \\
\text { COT1 }\end{array}$ & $\begin{array}{c}\text { LONG } \\
+ \\
\text { MOB2A }\end{array}$ & $\begin{array}{c}\text { LONG } \\
+ \\
\text { MOB2B }\end{array}$ \\
\hline $\begin{array}{c}\text { SHORT } \\
+ \\
\text { MOB2A }\end{array}$ & $\begin{array}{c}\text { SHORT } \\
+ \\
\text { MOB2B }\end{array}$ & $\begin{array}{c}\text { LONG } \\
+ \\
\text { PGADT7 }\end{array}$ \\
\hline $\begin{array}{c}\text { SHORT } \\
+ \\
\text { PGADT7 }\end{array}$ & $\begin{array}{c}\text { PGBKT7 } \\
+ \\
\text { LONG }\end{array}$ & $\begin{array}{c}\text { PGBKT7 } \\
+ \\
\text { SHORT }\end{array}$ \\
\hline
\end{tabular}
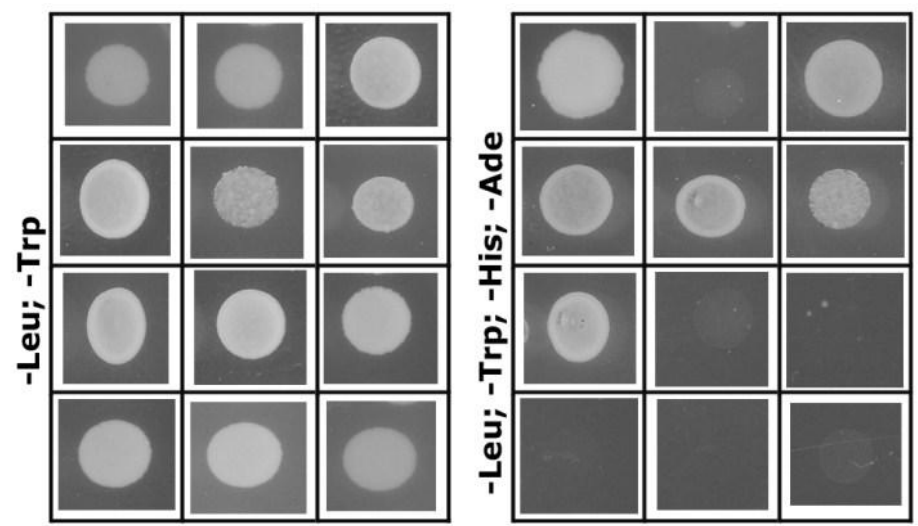

B
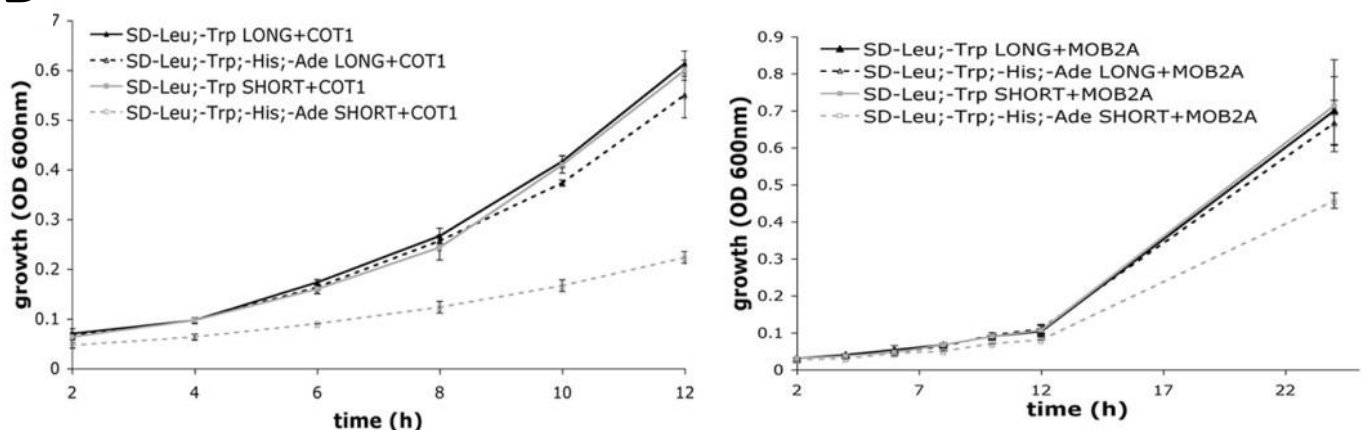

Figure 12: Analysis of COT1 homo-dimerization and COT1-MOB2 hetero-dimerization.

Genes were cloned into PGBKT7 or pGADT7. (A) Plasmids expressing the indicated proteins either as prey or bait alone were used as negative controls. pGBKT7-p53 (murine p53) and pGADT7-recT (SV40 large T antigene) fusions were used as positive control. (B) Growth curves of yeast cultures harboring the indicated two-hybrid plasmids in liquid medium.

\subsubsection{Activation of the NDR kinase COT1 by its upstream kinase POD6 regulates COT1 activity and membrane association}

For COT1 kinase activity the binding of both MOB2 proteins to the $\mathrm{N}$-terminus of COT1 is important and maybe resulting in autophosphorylation at residue Ser417 in the activation loop (Bichsel et al., 2004). In addition, phosphorylation at residue Thr589 in the hydrophobic motif by POD6 results in full NDR kinase activity (Bichsel et al., 2004, Hergovich et al., 2005, Jansen et al., 2006, Ziv et al., 2009). Moreover, both events are essential for the membrane association of COT1, but the mechanism of kinase transfer to the membrane is still 
unresolved. To clarify whether POD6 and/or the two MOB2 proteins are necessary for localizing COT1, a GFP-tagged COT1 construct was generated which complemented the $\Delta$ cot1 deletion. COT1-GFP localizes as a faint cap in growing hyphal tips and co-localizes with a vesicle accumulation in the subapical region, called the Spitzenkörper. COT1(T589A)-GFP did poorly complement $\Delta \cot -1$ and resulted in a strain with morphology and growth rate comparable to that of myc-cot-1(T589A) (data not shown). An ectopically integrated COT1(T589A)-GFP construct in a wild type background only localizes to the Spitzenkörper, but not as an apical membrane-associated cap. In conclusion, hydrophobic motif phosphorylation is required for membrane localization and functionality of COT1 (Figure 13A).

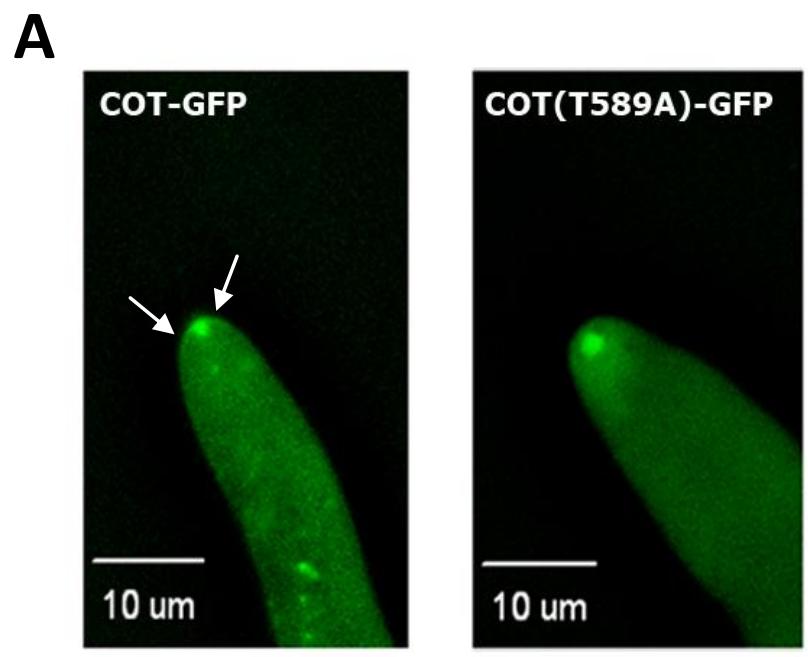

B
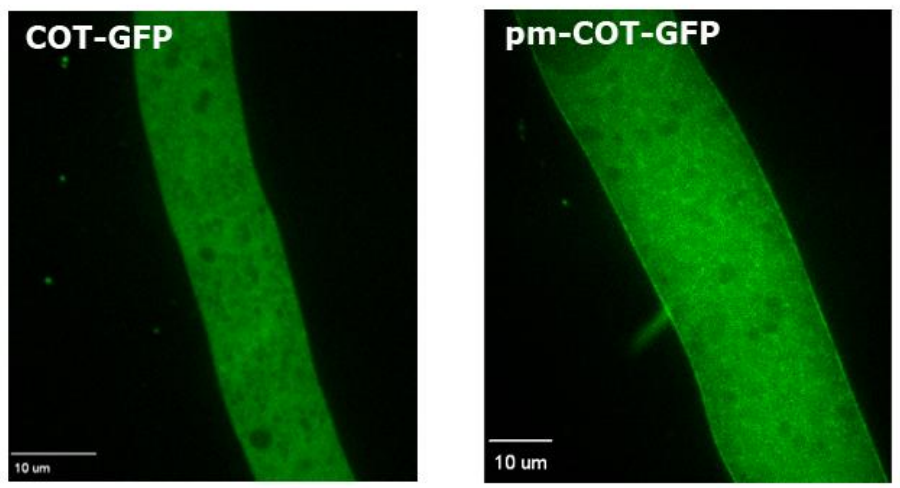

Figure 13: Localization pattern of COT1-GFP, COT1(T589A)-GFP and pm-COT1-GFP in wild type background.

(A) COT1-GFP localizes at the putative Spitzenkörper and as a faint-cap like accumulation at the hyphal tip (marked by arrows), whereas COT1(T589A)-GFP localizes only at the Spitzenkörper and not as an apical cap. (B) COT1-GFP localizes throughout the cytoplasm, whereas pm-COT1-GFP localizes at the whole plasma membrane and also throughout the cytoplasm. 
In a next step, an artificially membrane-targeted COT1 was generated by attaching a consensus motif for $\mathrm{N}$-terminal palmitylation/myristylation $(\mathrm{pm})$ to the $\mathrm{N}$-terminus of COT1. Ectopically expressed pm-COT1-GFP associated with the plasma membrane along the whole hypha (Figure 13B), confirming the suitability of the tag

In an earlier experiment, it was shown that the $\Delta$ pod-6 phenotype is partially suppressed by myc-COT1(T589E) (Figure 14A). Surprisingly the pm-myc-COT1(T589E) fully suppressed the spod-6 defect. Astonishing, also pm-myc-COT1 and pm-myc-COT1(T589A) fully complemented the $\Delta$ pod-6 defect. Further, the targeting of MOB2A to the plasma membrane using a pm-HA-mob-2a construct resulted in a full rescue of the $\Delta p o d-6$ phenotype (Figure 14B-C). Additionally, membrane-targeting of myc-COT1 or mycCOT(T589E) did not significantly change the in vitro activities of these COT1 variants in comparison to the non-targeted controls (personal communication by S. Maerz).

In conclusion, the phosphorylation of COT1 at threonine 589 by POD6 is not necessary if СОТ1 or its adaptor protein МОВ2A are directly localized to the plasma membrane. 
A

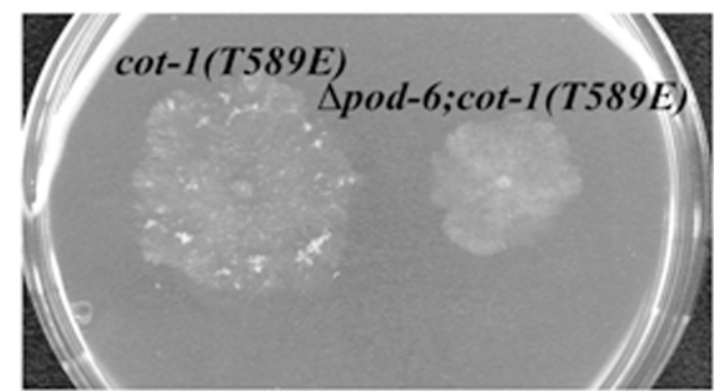

B
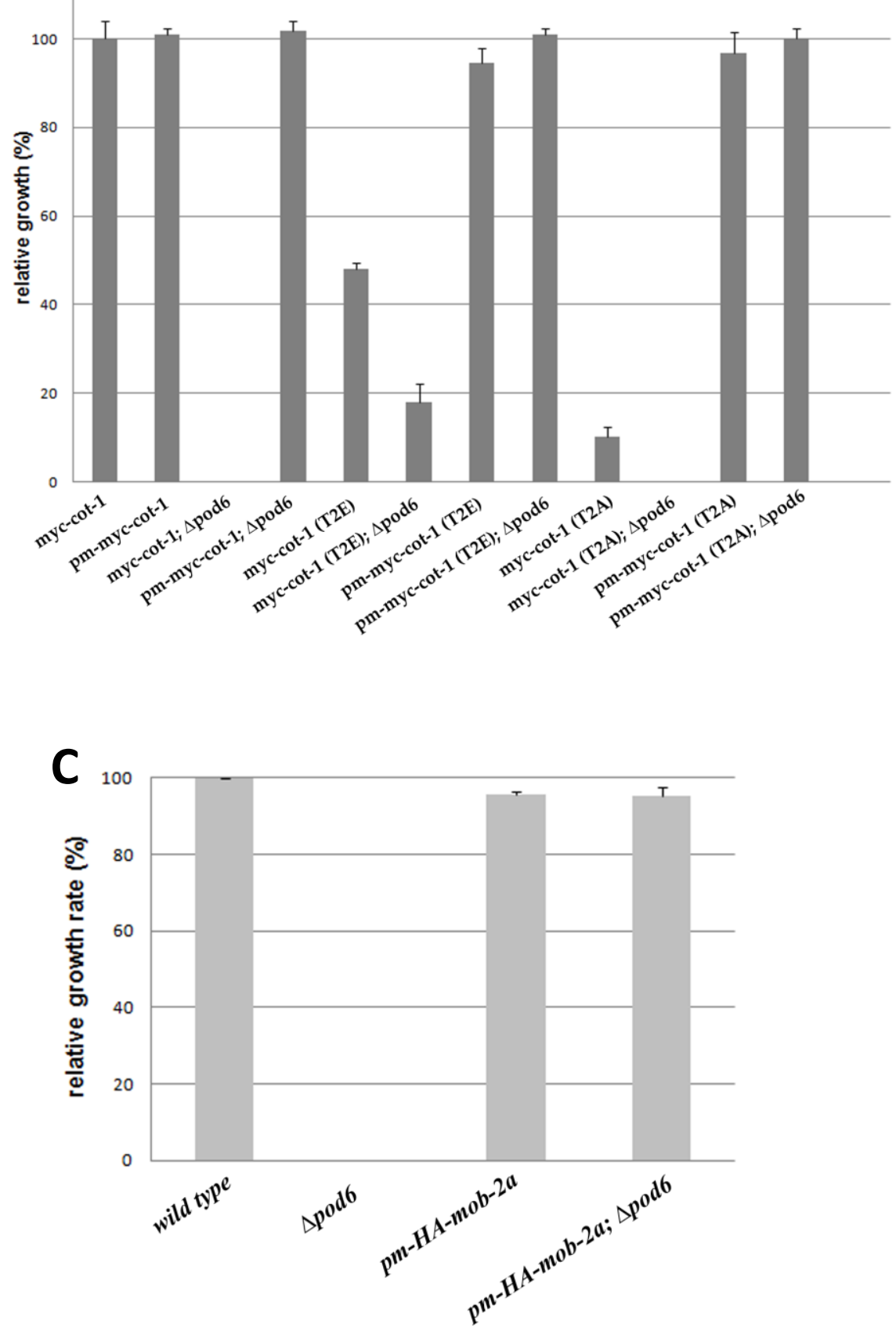

Figure 14: Thr589 phosphorylation of COT1 by POD6 is required for membrane association of COT1.

(A) Partial suppression of $\Delta$ pod-6 obtained by myc-COT1(T589E).

(B) All membrane-targeted myc-СОT1 constructs fully suppressed the $\Delta$ pod- 6 phenotype.

(C) Also the generated pm-HA-mob-2a construct fully suppressed the $\Delta p o d-6$ deletion mutant.

Conidia of indicated strains were incubated on VMM at $25^{\circ} \mathrm{C}$ 


\subsection{Hym 1 is part of the COT1 complex}

\subsubsection{HYM1 acts as a scaffold protein of COT1-POD6}

HYM1 belongs to the MO25 protein family, which is known to function as adaptors and master regulators of different Ste20 kinases and their effector kinases. To determine if HYM1 interacts with the NDR kinase COT1 and the Ste20 kinase POD6 in the fungus Neurospora crassa, constructs of these network components were generated, yeast twohybrid studies were performed and interactions were detected between COT1 or POD6 and HYM1 (Figure 15A). To verify these interactions, a myc-tagged HYM1 construct was cloned and ectopically expressed in a wild type $N$. crassa background. By performing an anti-MYCHYM1 immunoprecipitation experiment, both COT1 and POD6 (detected by anti-POD6 and anti-COT1 antibodies) were co-immunoprecipitated from extracts of the MYC-HYM1 strain, but not from wild type control (Figure 15B).

A

\begin{tabular}{|c|c|c|}
\hline $\begin{array}{c}\text { positive } \\
\text { control }\end{array}$ & $\begin{array}{c}\text { negative } \\
\text { control }\end{array}$ & $\begin{array}{c}\text { HYM1 } \\
+ \\
\text { PGADT7 }\end{array}$ \\
\hline $\begin{array}{c}\text { HYM1 } \\
+ \\
\text { COT1 }\end{array}$ & $\begin{array}{c}\text { HYM1 } \\
+\end{array}$ & $\begin{array}{c}+ \\
\text { POBK6 } \\
\text { COT1 } \\
\text { COT1 }\end{array}$ \\
\hline $\begin{array}{c}\text { pGBKT7 } \\
+ \\
\text { POD6 }\end{array}$ & & \\
\hline
\end{tabular}
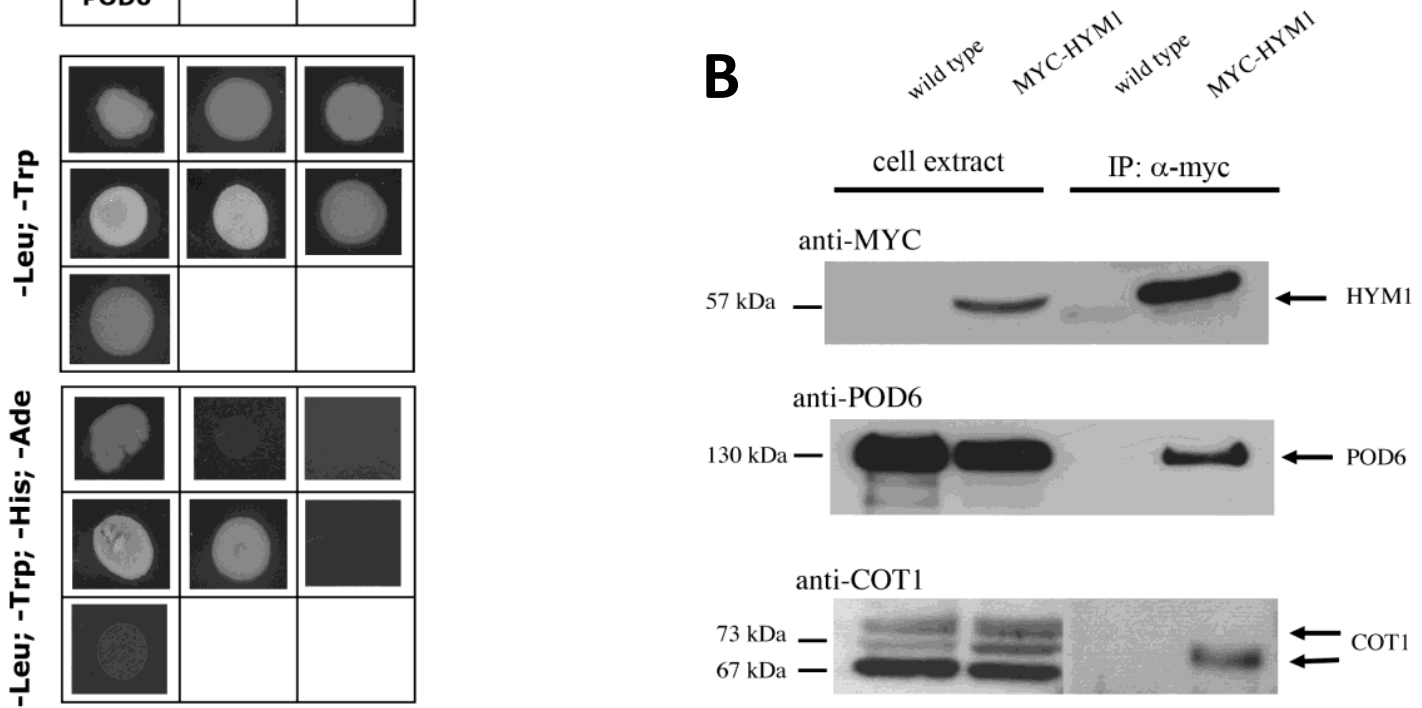

Figure 15: Interaction studies of HYM1 with COT1 and POD6.

(A) Genes were cloned into pGBKT7 either pGADT7 and plasmids expressing the indicated proteins either as prey or bait alone were used as negative controls. pGBKT7-53 (murine p53) and PGADT7-recT (SV40 large T antigene) fusions were used as positive control.

(B) Immunoprecipitation experiment of myc-tagged HYM1 recovered POD6 and COT1. 
To further investigate the role of HYM1 as a potential regulator of COT1 and POD6, formation of a stable COT1-POD6 complex was analyzed in $\Delta h y m-1$. Therefore, an HA-pod-6 construct was generated by amplifying the $5^{\prime}$ and pod- 6 regions and fusing both via PCR by their partial overlapping HA-tag sequence and final generating the HA-pod-6 replacement cassette with Ndel restriction sites at each end. The HA-pod-6 replacement cassette was excised and transformed into pod-6(ts); $\Delta$ mus52::bar; his-3. The final strain with the HAtagged POD6 under the endogenous promoter was crossed with a strain containing a myc::cot-1 gene under the endogenous promoter (Ziv et al., 2009). In a further step, the strain with the HA-tagged POD6 and myc-tagged COT1 was crossed with $\Delta h y m-1$.

Subsequently, an immunoprecipitation with myc-antibody against myc-tagged COT1 was performed to detect co-purified HA-tagged POD6 by a Western blot with HA-antibody.

Unfortunatly, it was not possible to co-purify POD6 and COT1 in the hym-1 deletion background in contrast to the control strain containing myc-tagged COT1 and HA-tagged POD6 (Figure 16). So it seems that the interaction between COT1 and POD6 is not stable in the absence of the scaffold protein HYM1.

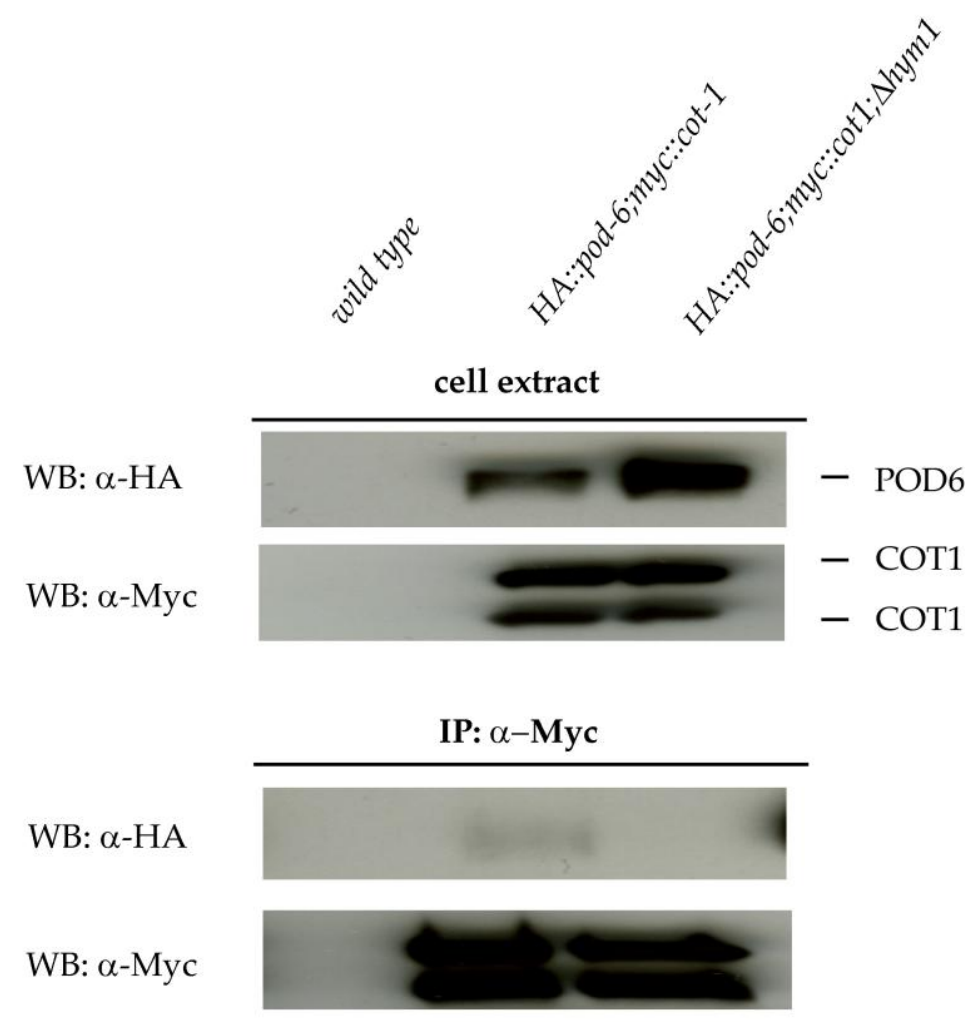

Figure 16: Co-immunoprecipitation of myc-tagged COT1 and HA-tagged POD6 in a hym-1 deletion background. 


\subsubsection{The HYM1-COT1 complex localizes to septa and the hyphal apex}

Based on yeast two-hybrid studies and co-immunoprecipitation analysis we supposed a colocalization between COT1 and HYM1. Therefore GFP-tagged constructs of both proteins were generated and overlapping localization patterns were found. GFP-tagged COT1 under the endogenous promoter localizes at the final septa, as an accumulation in the putative Spitzenkörper as well as a faint cap-like accumulation in the apical plasma membrane in growing hyphal tips (see also section 5.1.2) (Figure 17).
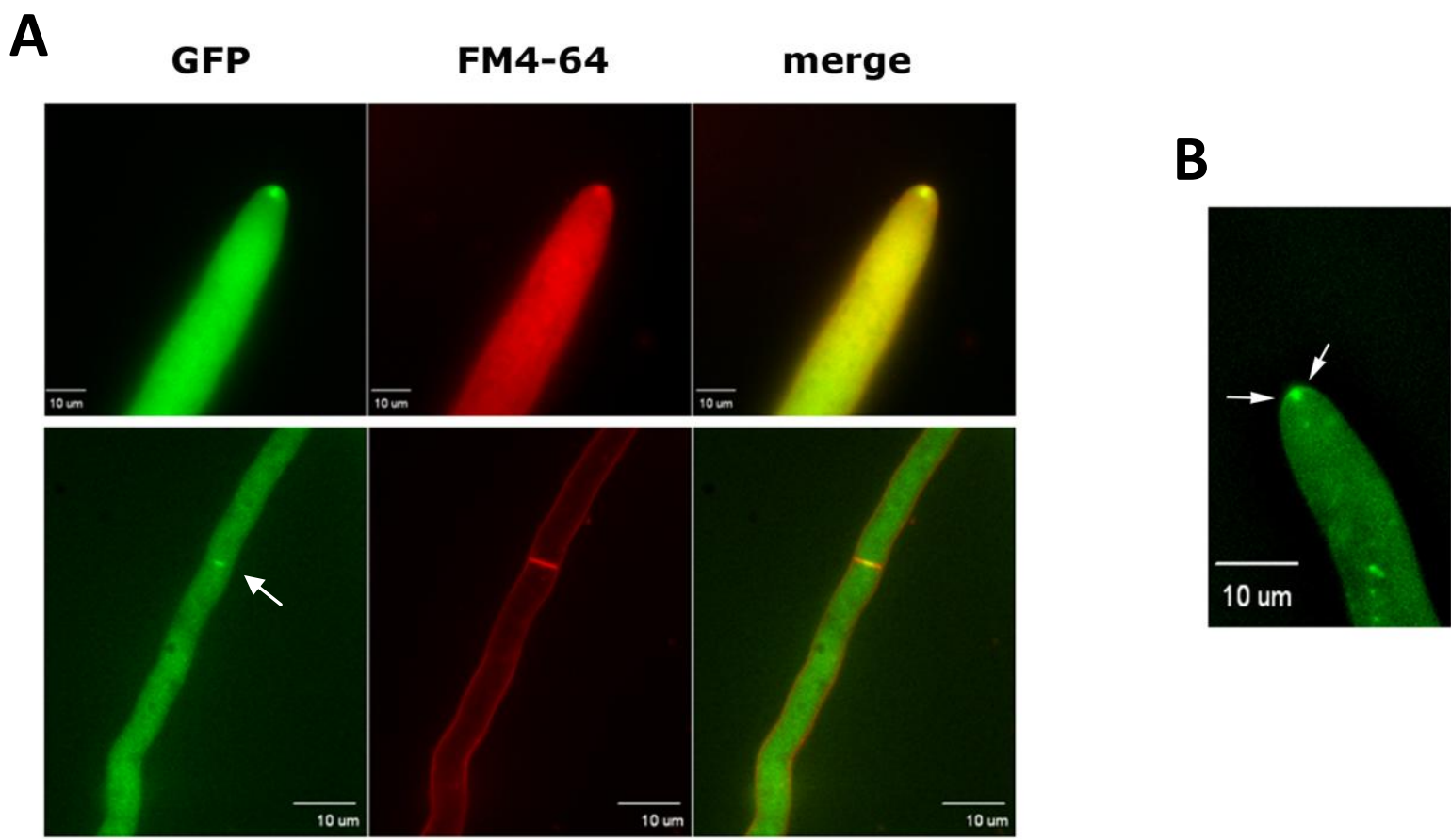

Figure 17: Localization pattern of GFP-tagged COT1.

(A) COT1-GFP localizes at the final septa (indicated by marked arrow), as an accumulation in the putative Spitzenkörper and as a faint cap-like accumulation in the apical plasma membrane. Plasma membrane and Spitzenkörper were stained with FM4-64. Scale bars are 10 $\mu \mathrm{m}$.

(B) COT1-GFP localizes at the apical membrane marked by arrows. Bars=10 $\mu \mathrm{m}$.

The GFP-tagged HYM1 under the endogenous promoter localizes at forming septa and at the putative Spitzenkörper (Figure 18). In summary, a partial co-localization pattern for HYM1 and COT1 was observed. 

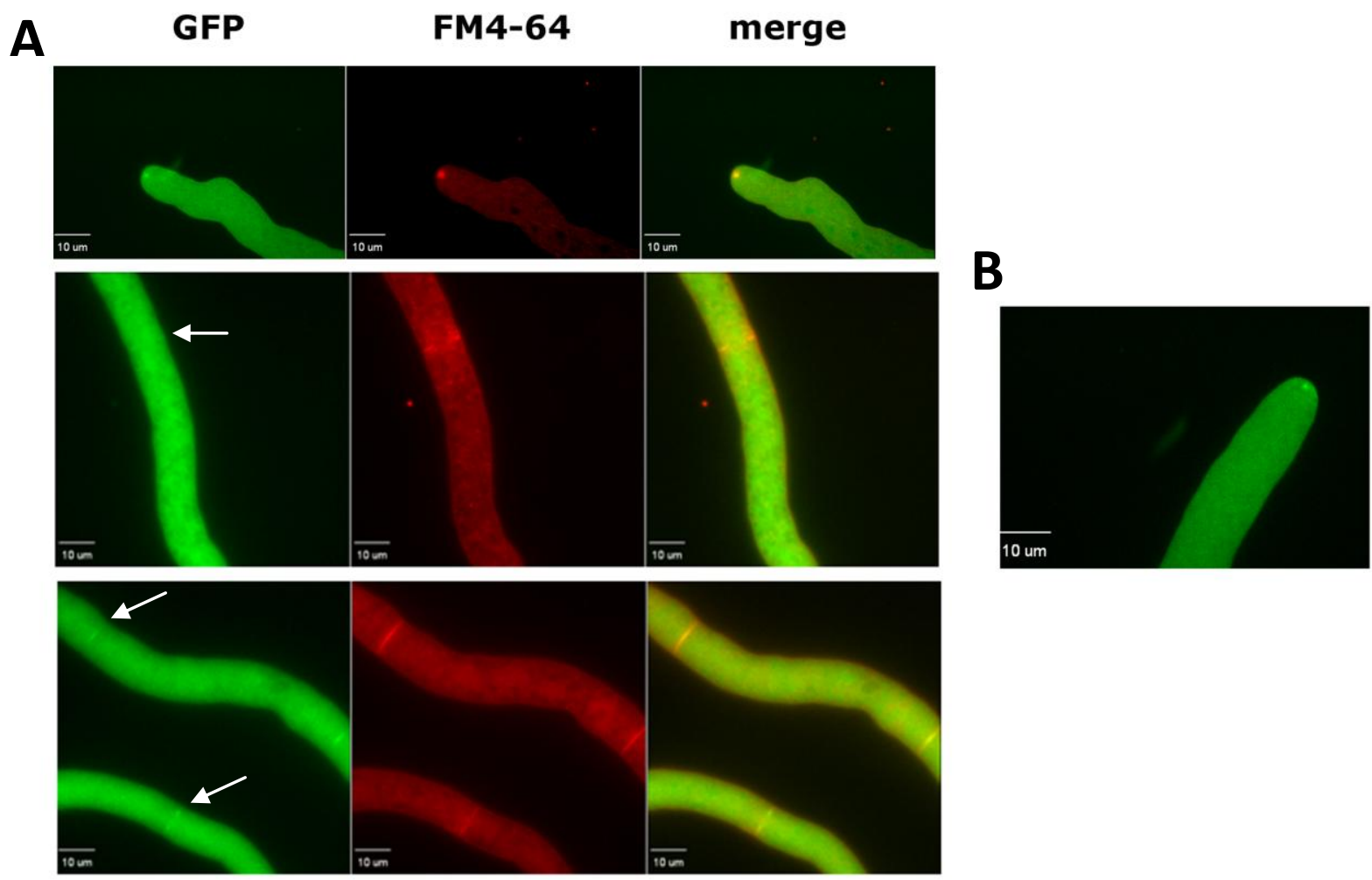

Figure 18: Localization pattern of GFP-tagged HYM1.

(A) gfp::hym1 localizes at forming septa (indicated by marked arrows) and in the putative Spitzenkörper. Plasma membrane and Spitzenkörper were stained with FM4-64. Scale bars are 10 $\mu \mathrm{m}$.

(B) HYM1-GFP localizes as an accumulation in the Spitzenkörper (marked by arrows). Bars=10 $\mu \mathrm{m}$

\subsection{3 $\Delta$ hym-1 displays phenotypic defects of MAK2 pathway mutants}

Mutations in the central components of the COT1 signaling pathway cot-1, pod-6 and mob-2 genes show significant defects, such as cessation of hyphal elongation and the formation of massive amounts of extension-arrested new tips along the entire cell (Maerz et al., 2009, Seiler et al., 2006, Yarden et al., 1992). Surprisingly, the deletion of hym-1 does not show this "barbed wired" phenotype, but displayed characteristic vegetative and developmental defects: highly irregular "zig-zag" hyphal morphology, reduced aerial mycelium and derepressed conidiation, the inability to develop protoperithecia as well as hyphal and germling fusion defects (Figure 19). Interestingly, the phenotypic characteristics of $\Delta h y m-1$ were identical to mutants of the MAK2 MAP kinase pathway. 
A
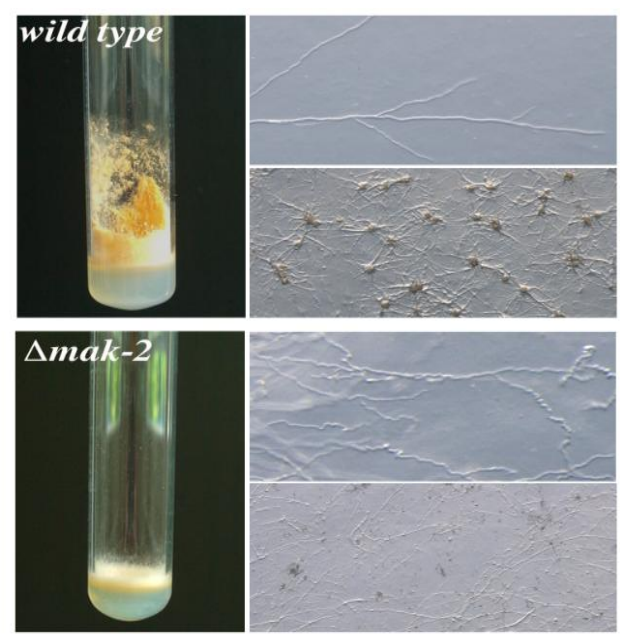

$\Delta h y m-1$
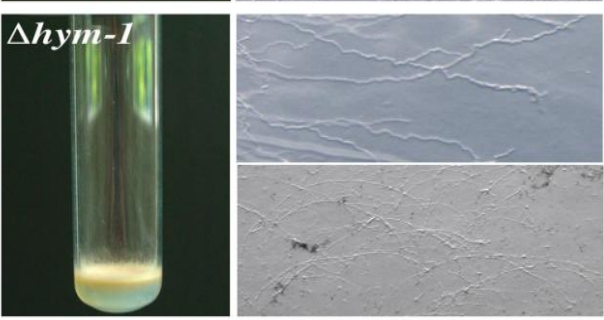

B
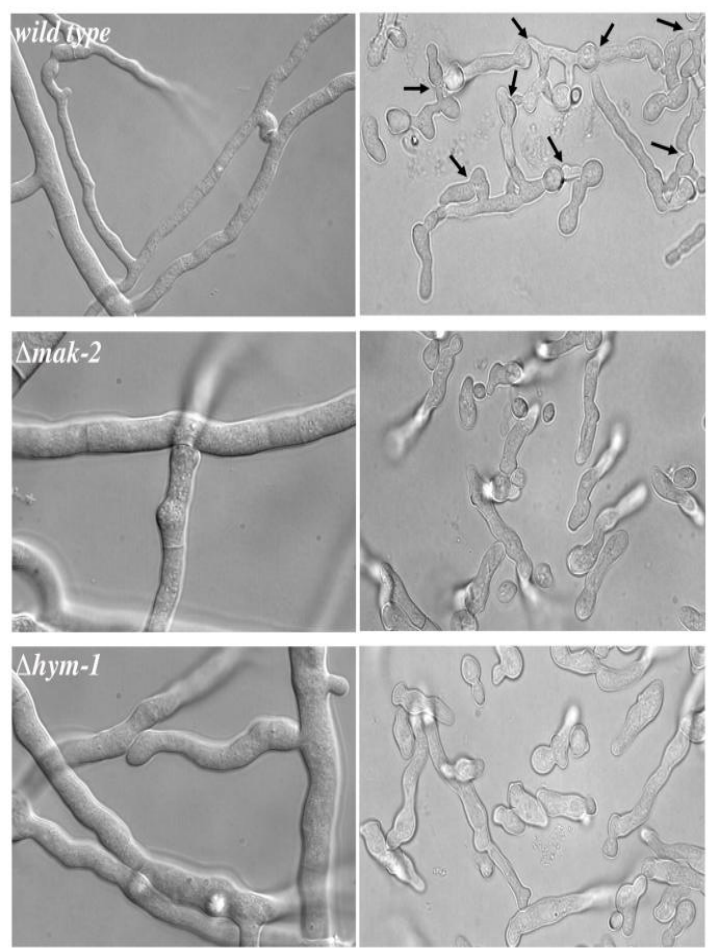

Figure 19: Phenotypical characterization of $\Delta h y m-1$ and $\Delta m a k-2$.

(A) $\Delta$ hym-1 and $\Delta$ mak-2 mutants compared to wild type regarding colony morphology (growth for 5 days on minimal medium; bar $=1 \mathrm{~cm}$ ), hyphal morphology (on minimal medium, bar $=\mathbf{5 0} \mathrm{mm}$ ), and protoperithecia formation (growth for 7 days on cornmeal medium, bar $=300 \mathrm{~mm}$ ) was determined.

(B) HYM1 and MAK2 are required for vegetative cell fusion. Hyphal fusion (left images; bar = $10 \mathrm{~mm}$ ) and germling fusion (right images; bar $=5 \mathrm{~mm}$ ) in wild type, $\Delta h y m-1$ and $\Delta m a k-2$ cultures were assessed by light microscopy. Fusion events are indicated by arrows. Cell fusion was not observed in $\Delta h y m-1$ and $\Delta m a k-2$.

So far, HYM1 acts as a potential adaptor protein for the kinases COT1 and POD6, but the deletion phenotype is more similar to the mak-2 pathway mutants. These results indicate an interaction between the COT1 signaling and the MAK2 MAP kinase pathway via HYM1.

To further analyze the role of HYM1 in the MAK2 MAP kinase cascade, a MAK2 activity assay in a $\Delta h y m-1$ background was performed. Therefore, the indicated strains were grown in liquid medium under normal growth conditions and MAP kinase stress-induced (with $\mathrm{H}_{2} \mathrm{O}_{2}$ ) conditions. Quantified cell extracts were used for SDS gel electrophoresis followed by Western blot and immunodetection with p42/p44-phospho-specific MAP kinase antibody against activated MAP kinases. The resulting blots showed an abolished MAK2 activity in $\Delta$ hym-1, whereas the MAK1 activity is not affected indicating the importance of HYM1 for the MAK2 signaling (Figure 20). 

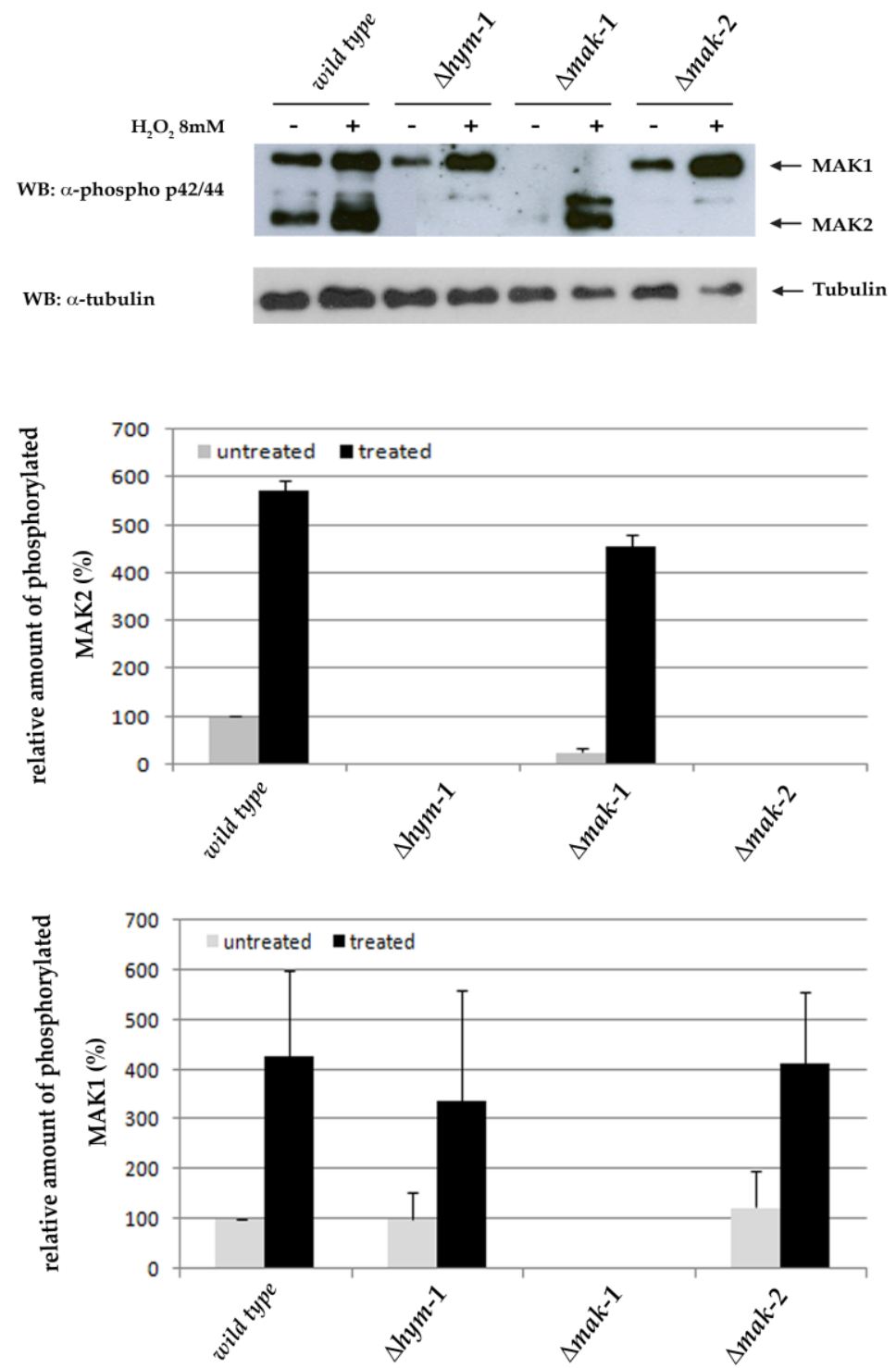

Figure 20: MAK2 activity is abolished in $\Delta$ hym-1.

Total soluble protein ( $75 \mu \mathrm{g}$ per lane) was extracted from the indicated strains grown in the presence or absence of $8 \mathrm{mM} \mathrm{H}_{2} \mathrm{O}_{2}$. The strains were grown at $25^{\circ} \mathrm{C}$. The blot was probed with anti-phospho-p42/p44 MAP kinase antibody to detect activated MAK1 respectively MAK2. To confirm equal loading the blot was stripped and re-probed with $\alpha$-tubulin antibody.

In Neurospora crassa, the MAK2 kinase cascade consists of three MAP kinases, NRC1 as MAP kinase kinase kinase, MEK2 as MAP kinase kinase and MAK2 as the respective MAP kinase. To determine potential interactions between HYM1 and the components of the MAK2 pathway, constructs for co-immunoprecipitation studies in the fungus were generated. Therefore, a nic-3 strain expressing myc-tagged HYM1 was fused with a trp-1 strain expressing HA-tagged MAK2 or FLAG-tagged NRC1. The forced heterokaryons which were able to grow on minimal medium by complementation of the individual auxotrophies were 
used for the co-IP experiment and it was possible to co-purify HYM1 with MAK2 and NRC1 (Figure 21A). Moreover, a yeast two-hybrid experiment with HYM1 (NCU03576), MAK2 (NCU02393) and NRC1 (NCU06182) was executed, but no interactions were detected (Figure 21B). To demonstrate the functionality of the yeast two-hybrid constructs, expected interactions between the three MAP kinases were verified (Figure 21C). In other words, HYM1 interacts with proteins of the MAK2 pathway but not in a direct manner.
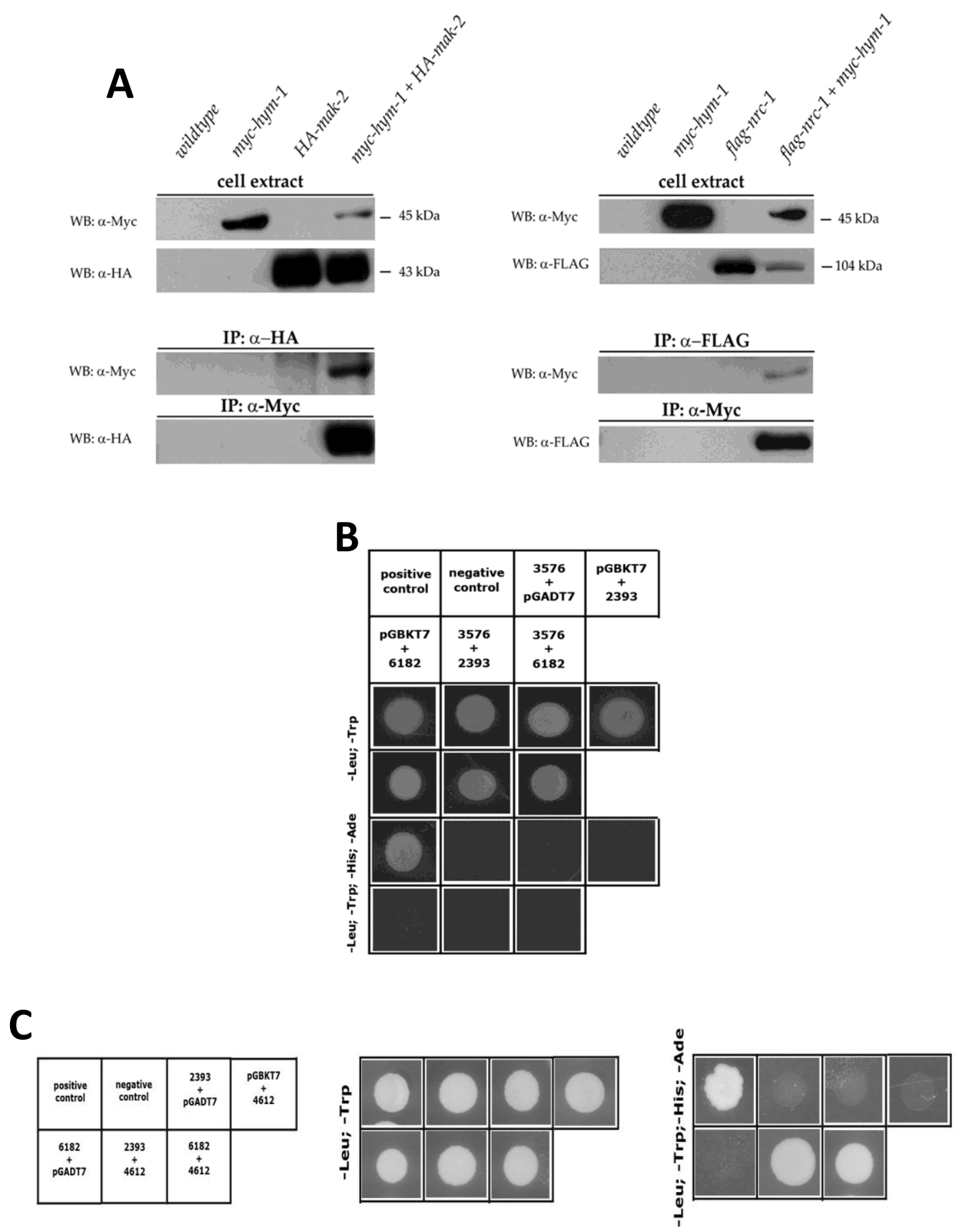

Figure 21: Interaction studies of HYM1 with proteins of the MAK2 MAP kinase cascade.

(A) Co-immunoprecipitation studies in $N$. crassa show interactions between HYM1 and MAK2 respectively NRC1. 
(B) Yeast two-hybrid analysis between HYM1 and the MAP kinases NRC1, MEK2 and MAK2 showed no interactions.

(C) Interactions between the proteins of the MAK2 MAP kinase cascade (MAK2 (NCU02393); STE7 (NCU04612); NRC1 (NCU06182)) by yeast two-hybrid as a control.

\subsubsection{HYM1 functions as a linker protein for the MAK2 pathway}

My previous experiments have shown that HYM1 is important for MAK2 activity and interacts with the MAK2 cascade (although not directly). To determine a hierarchical order between HYM1 and the proteins of the MAK2 cascade, dominant-active (DA) constructs of the triple MAP kinase NRC1 were generated according to published substitutions (Maleri et al., 2004, Mueller et al., 2003, Zhao et al., 2006). Two NRC1 variants, one with a point mutation which leads to an aa substitution from proline 448 to serine and second a deletion of aa 41 to 590 were cloned. The DA constructs were first transformed in a wild type background to determine the expression levels of the proteins. Both constructs were similar expressed verified in a Western blot using FLAG-antibody. In comparison to the wild type, the unmutated full length NRC1 protein displayed a normal growth rate with normal aerial mycelium, protoperithecia formation and the ability to perform cell fusion. Both dominantactive constructs showed also a normal growth, formation of aerial mycelium and cell fusion, but the ability to form protoperithecia was delayed. In addition, the dominant-active constructs of NRC1 showed an increased MAK2 activity in contrast to the unmutated full length of NRC1 determined by Western blot using p42/p44-phospho-specific antibody (Table 6, Figure 22).

Furthermore, the strains expressing the dominant-active versions in wild type background were crossed with the $\Delta n r c-1$ strain to determine their functionality. In contrast to $\Delta n r c-1$, the construct with the unmutated full length NRC1 showed normal growth, formation of aerial mycelium and maintained the ability to form protoperithecia and cell fusion.

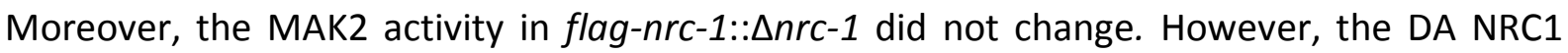
constructs did not fully complement $\Delta n r c-1$. The dominant-active construct with a point mutation rescued the growth rate, the formation of aerial mycelium and partial the ability to form protoperithecia, whereas hyphal and germling fusion were not complemented. The second DA construct harboring a deletion did not rescued any morphological or developmental defects of $\Delta n r c-1$ suggesting that this construct is not functional (Table 6; 
Supplementary Figure 1). As expected, both dominant-active NRC1 constructs showed hyperactive MAK2 activity in $\Delta n r c-1$ (Figure 22).

In an additional experiment, the DA NRC1 constructs were crossed with $\Delta h y m-1$. So far, we only obtained the flag-nrc-1 ${ }^{\text {P448s }}$; $\Delta h y m-1$. A rescue of the $\Delta h y m-1$ growth rate was observed, but the MAK2 activity is still abolished (Table 6).

Therefore, one can assume that HYM1 is not acting upstream of the MAK2 kinase cascade.

Table 6: Phenotypical characterization of dominant-active NRC1 constructs in a his-3, $\Delta$ nrc-1 and $\Delta h y m-1$ background.

\begin{tabular}{|c|c|c|c|c|}
\hline & his-3 & flag-nrc-1::his-3 & flag-nrc-1 ${ }^{\text {P4a85 }}$ ::his-3 & flag-nrc-1 $1^{\text {deletion aa41-590 }}:$ his-3 \\
\hline Growth rate & $3,8( \pm 0,2)$ & $3,2( \pm 0,1)$ & $3,3( \pm 0,2)$ & $3,8( \pm 0,2)$ \\
\hline Aerial mycelium & yes & yes & yes & yes \\
\hline PP formation & yes & yes & yes (delayed) & yes (delayed) \\
\hline Germling fusion & yes & yes & yes & yes \\
\hline \multirow[t]{2}{*}{ MAK2 activity (\%) } & $100 \%$ & $100 \%$ & $1000 \%$ & $1000 \%$ \\
\hline & $\Delta n r c-1$ & flag-nrc-1::Anrc-1 & flag-nrc-1 ${ }^{p 4485}:: \Delta n r c-1$ & flag-nrc-1 ${ }^{\text {aeletion } a{ }^{2} 41=590}:: \Delta n r c-1$ \\
\hline Growth rate & $1,6( \pm 0,2)$ & $3,3( \pm 0,1)$ & $3,1( \pm 0,2)$ & $1,6( \pm 0,2)$ \\
\hline Aerial mycelium & no & yes & yes (reduced conidia) & no \\
\hline PP formation & no & yes & very few & no \\
\hline Germling fusion & no & yes & no & no \\
\hline \multirow[t]{2}{*}{ MAK2 activity(\%) } & $0 \%$ & $0 \%$ & $1000 \%$ & $1000 \%$ \\
\hline & $\Delta h y m-1$ & & flag::nrc-1 ${ }_{1}^{p 4485}:: \Delta h y m-$ & \\
\hline Growth rate & $1,4( \pm 0,1)$ & & $2,9( \pm 0,2)$ & \\
\hline Aerial mycelium & no & & no & \\
\hline PP formation & no & & no & \\
\hline Germling fusion & no & & no & \\
\hline MAK2 activity & 0 & & 0 & \\
\hline
\end{tabular}




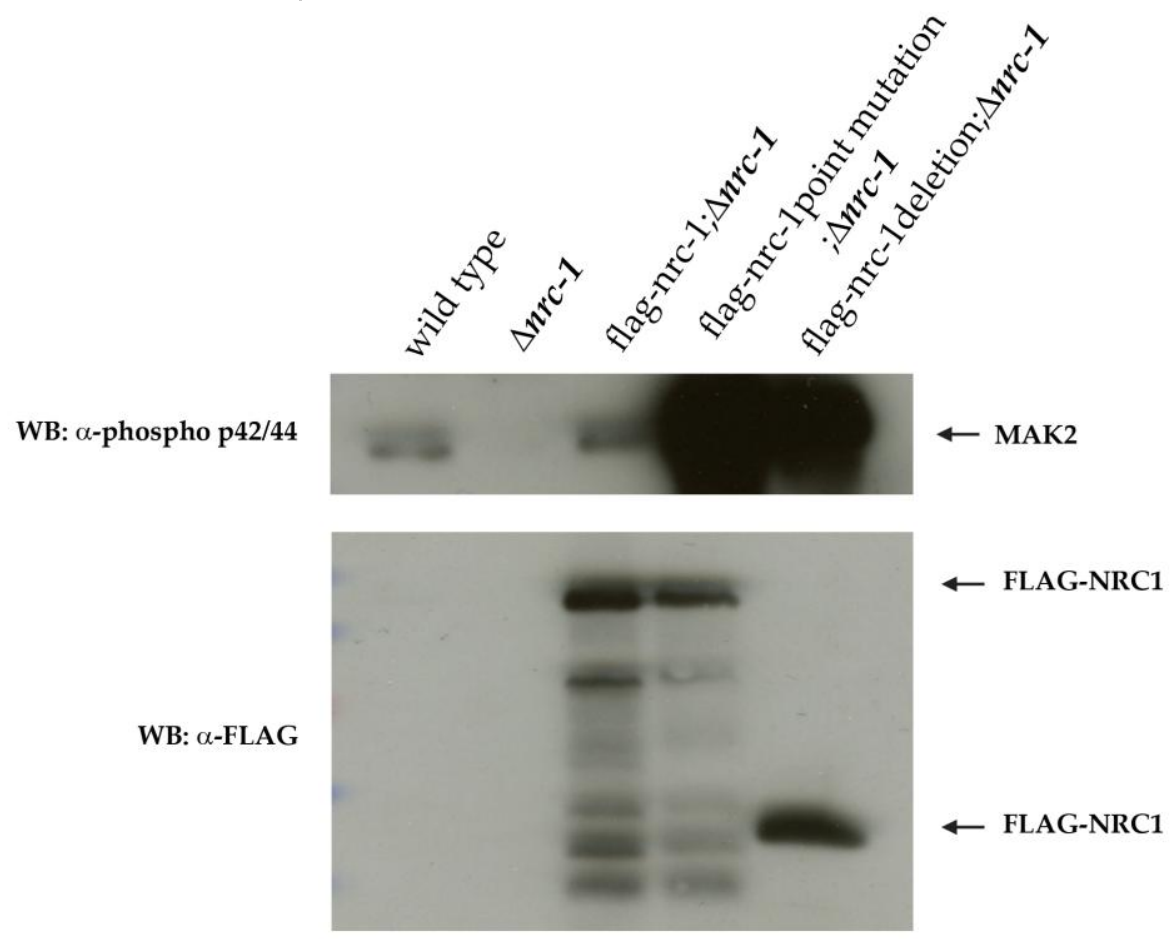

Figure 22: Immunoblot illustrating the increased MAK2 phosphorylation levels of DA NRC1 constructs in contrast to wild type NRC1 in the nrc-1 deletion background.

Actually, the different experiments attest that the dominant-active versions of NRC1 are hyperactive and might be constitutively active due to the literature. In addition, the generation of adominant-active construct of MEK2, the MAK2 kinase kinase, is in progress.

\subsubsection{Characterization of NDK1 and GCK1, potential HYM1 interaction partners}

As indicated by my previous yeast two-hybrid and co-IP experiments, HYM1 interacts with MAK2 components only in an indirect manner. In order to identify proteins that may connect HYM1 with the MAK2 pathway components, a yeast two-hybrid screen with HYM1 as bait was performed to identify further potential interaction partners of HYM1. In addition to proteins of general biosynthesis and metabolism that were regarded as likely false positives, two potential interaction partners, a NDPK (nucleoside-diphosphate kinase) and a GCK (germinal centre kinase) were detected (Figure 23A). To verify these interactions HYM1 (NCU03576), GCK1 (NCU00772) and NDK1 (NCU04202) constructs were generated for coimmunoprecipitation experiments. Interactions between HYM1 and NDK1 as well as HYM1 and GCK1 were detected (Figure 23B). 
A
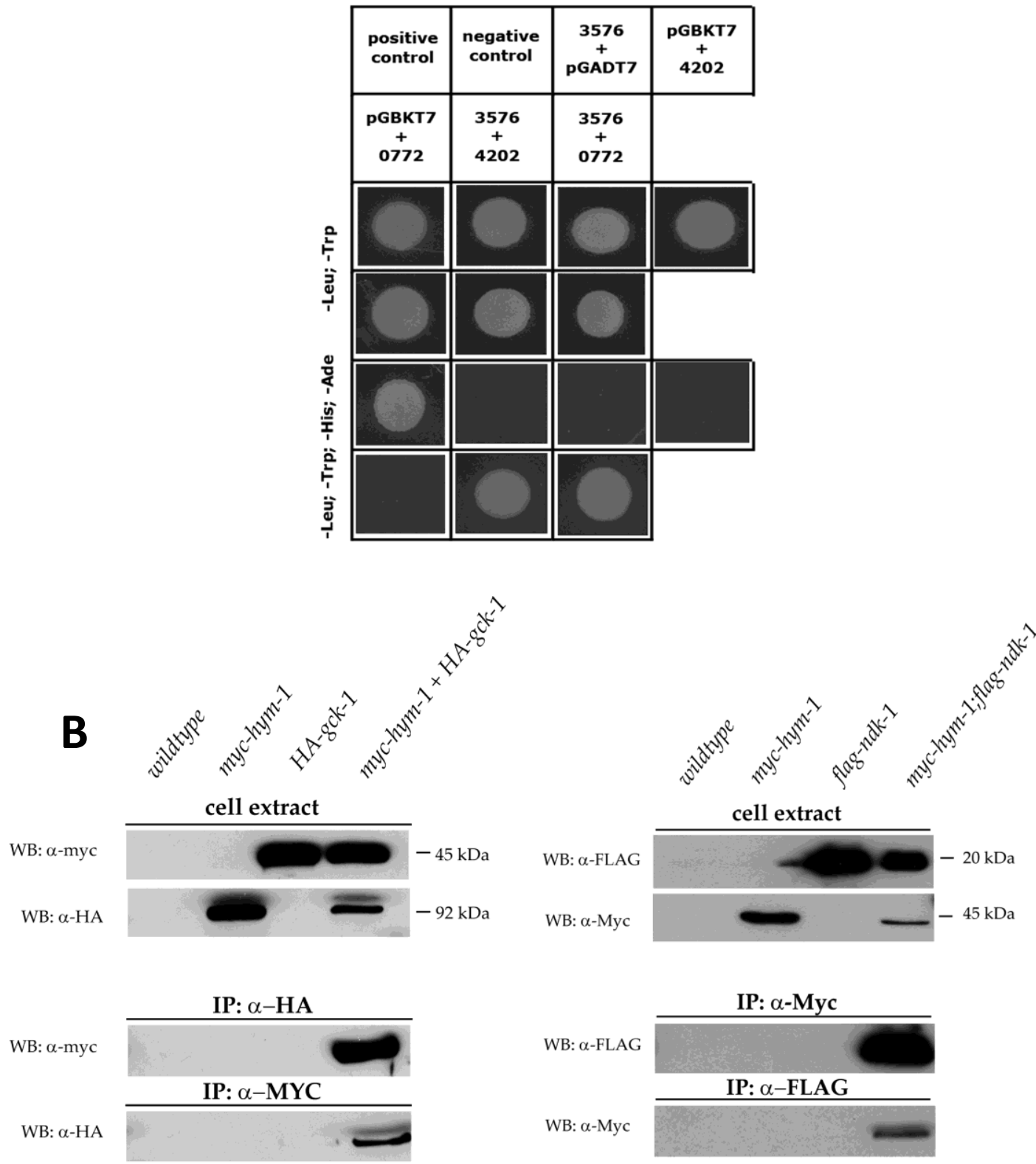

Figure 23: Interaction studies of HYM1 with NDK1 and GCK1.

(A) Genes of hym-1, ndk-1 and gck-1 were cloned into pGBKT7 either pGADT7 and plasmids expressing the indicated proteins either as prey or bait alone were used as negative controls. pGBKT7-53 (murine p53) and pGADT7-recT (SV40 large T antigene) fusions were used as positive control.

(B) Co-immunoprecipitation experiments between HYM1 and NDK1 and HYM1 with GCK1.

Next, these two candidate proteins were tested for their interactions with the MAK2 pathway components NRC1, MEK2 and MAK2. Yeast two-hybrid experiments exhibited an interaction of NDK1 with NRC1 and no interaction with MEK2 and MAK2. In addition, 
interactions of GCK1 with NRC1 and with MEK2 an no interaction with MAK2 were observed by yeast two-hybrid studies (Figure 24 ).

A

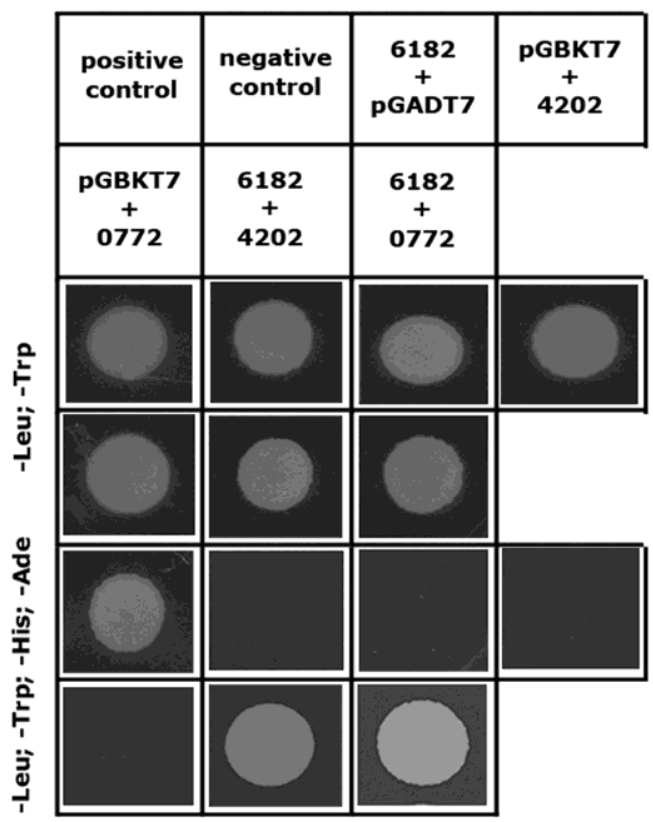

B
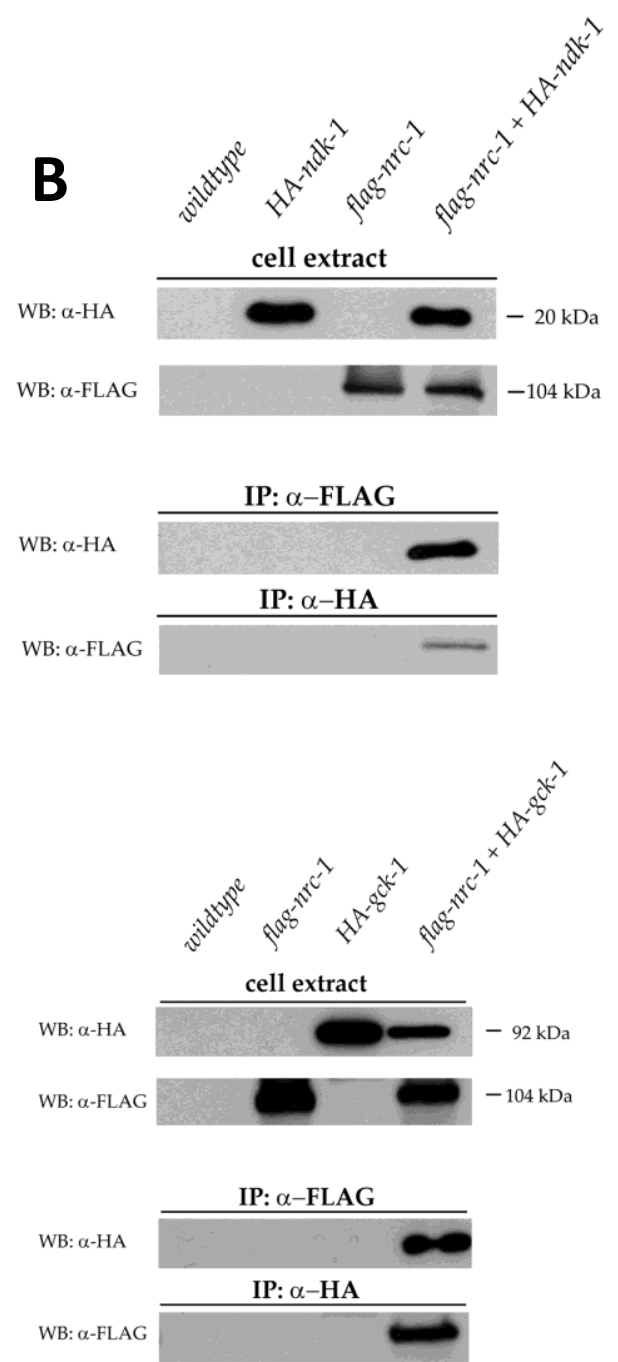

Figure 24: Interactions of NRC1 with NDK1 and GCK1.

(A) Yeast two-hybrid analysis between GCK1 and NDK1 with NRC1 showed interactions. The yeast twohybrid constructs were co-expressed as fusion proteins to the GAL4 DNA binding (BD) or activation (AD) domain in the reporter yeast strain AH109. Growth of colonies in medium lacking adenine, histidine, leucine and tryptophane reveal interactions. Fusion proteins for positive and negative control were expressed under the same promoter.

(B) Co-immunoprecipitation experiments between NRC1 and NDK1 respectively GCK1.

In summary, on the one hand interactions between HYM1 and the MAK2 kinase cascade and on the other interactions between the HYM1-interacting proteins GCK1 and NDK1 with the MAK2 kinase cascade were found suggesting that these proteins acting together in one complex (Figure 25). 


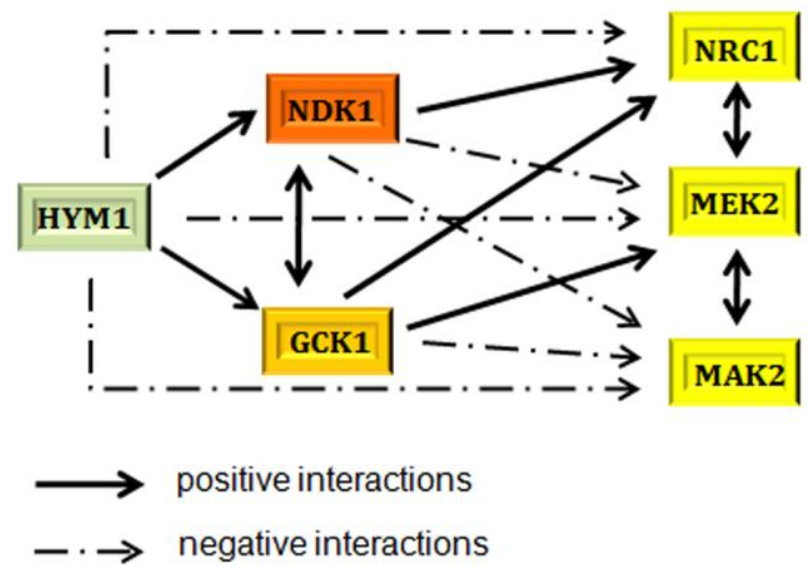

Figure 25: Overview about positive and negative interactions between HYM1, NDK1, GCK1 and proteins of the MAK2 cascade verified by yeast two-hybrid studies.

For the hym-1 and mak-2 deletion strains the same morphological and developmental defects were observed. Regarding $\Delta n d k-1$ and $\Delta g c k-1$ totally different phenotypes could be described.

$\Delta n d k-1$ shows a knobby hyphal morphology and exhibit a slow growth rate. The strain is able to perform vegetative cell fusion and to form protoperithecia. Compared to the wild type, the MAK2 activity is decreased under normal conditions and oxidative stress.

In contrast, $\Delta g c k-1$ displays a normal growth rate and a normal hyphal morphology identical to the wild type. Moreover, $\Delta g c k-1$ is also able to form protoperithecia, to perform vegetative cell fusion and the MAK2 activity is increased under normal growth and stressinduced conditions in contrast to the wild type.

In addition, a double deletion strain of $n d k-1$ and $g c k-1$ was generated to determine a hierarchical order of these genes. The $\Delta n d k-1 ; \Delta g c k-1$ deletion strain shows also a knobby hyphal morphology with a slow growth rate similar to $\Delta n d k-1$. The double knockout is able to form protoperithecia and to perform vegetative cell fusion. The MAK2 activity is decreased under normal growth conditions and is no longer inducible by osmotic stress (Figure 26). In conclusion, the deletion strains of the proteins of the MAK2 cascade, NDK1, HYM1 and GCK1 show pleiotrophic effects indicating different functions in one network. 
A
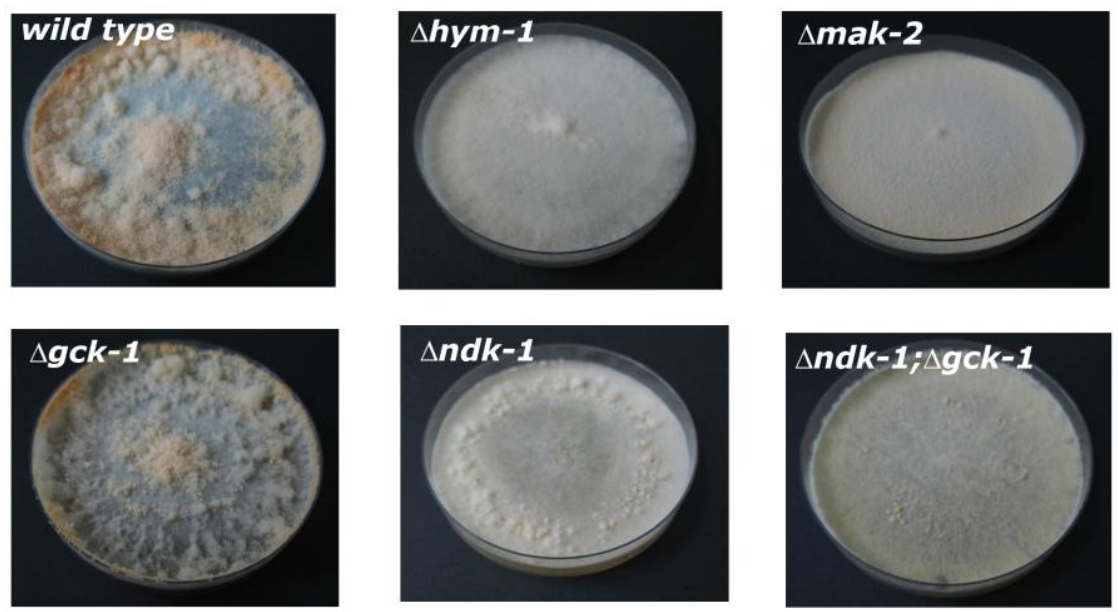

B

\begin{tabular}{|c|c|c|c|c|c|c|}
\hline & wild type & $\Delta m a k-2$ & $\Delta h y m-1$ & $\Delta g c k-1$ & $\Delta n d k-1$ & $\Delta g c k-1 ; \Delta n d k-1$ \\
\hline $\begin{array}{l}\text { Growth rate } \\
\text { (cm/day) }\end{array}$ & $3,9( \pm 0,2)$ & $1,6( \pm 0,1)$ & $1,4( \pm 0,1)$ & $3,2( \pm 0,1)$ & $1,2( \pm 0,2)$ & $1,7( \pm 0,4)$ \\
\hline Hyphal morphology & wild type & knobby & knobby & wild type & knobby & knobby \\
\hline $\begin{array}{l}\text { Vegetative cell } \\
\text { fusion }\end{array}$ & yes & no & no & yes & yes & yes \\
\hline $\begin{array}{l}\text { Arial mycelium and } \\
\text { conidiation }\end{array}$ & wild type & $\begin{array}{l}\text { short and } \\
\text { profuse }\end{array}$ & $\begin{array}{l}\text { short and } \\
\text { profuse }\end{array}$ & wild type & $\begin{array}{l}\text { poor and } \\
\text { clumped }\end{array}$ & poor and clumped \\
\hline $\begin{array}{l}\text { Protoperithecia } \\
\text { formation }\end{array}$ & yes & no & no & yes & yes & yes \\
\hline Perithecia formation & yes & no & no & yes & yes & yes \\
\hline $\begin{array}{l}\text { Ascospore } \\
\text { morphology and } \\
\text { viability }\end{array}$ & $\begin{array}{l}\text { normal, } \\
\text { viable }\end{array}$ & $\begin{array}{l}\text { normal, } \\
\text { inviable }\end{array}$ & $\begin{array}{l}\text { normal, } \\
\text { inviable }\end{array}$ & no ascospore & $\begin{array}{c}\text { normal, } \\
\text { viable }\end{array}$ & \\
\hline
\end{tabular}

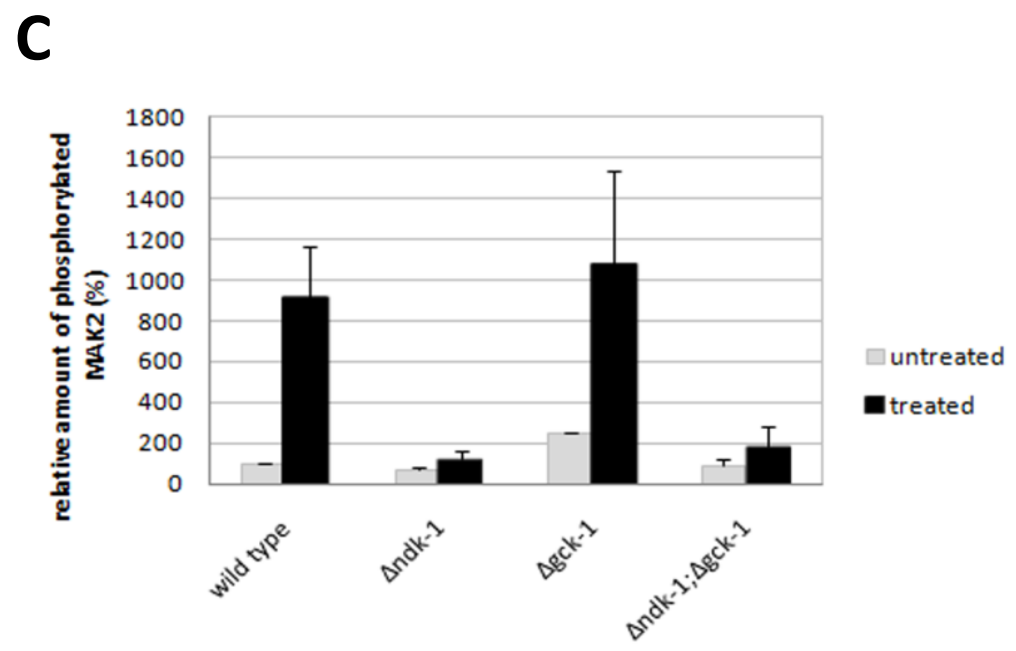

Figure 26: Phenotypic characterization of the indicated deletion strains.

(A) Colony morphology of the indicated strains grown at $25^{\circ} \mathrm{C}$ on minimal media plates is shown.

(B) Overview of the phenotypic characterization during vegetative growth and (a)sexual development.

(C) Summary of relative MAK2 activity levels in the different strains. Cell lysates prepared from unstressed and stressed $\boldsymbol{N}$. crassa cultures were subjected to SDS-PAGE and Western blotting. Phosphorylated MAK2 was detected using a phospho-specific-p42/p44-MAP kinase antibody. MAK2 phosphorylation levels were quantified by densiometric analysis of MAK2 band intensity and normalized to the values obtained for the unstressed wild type. 
In addition to the yeast two-hybrid experiments for genetic interaction studies between HYM1 and the MAK2 cascade and co-immunoprecipitation experiments GFP-tagged constructs of GCK1 and NDK1 were generated to determine the localization pattern. Therefore both kinases were tagged C-terminally with GFP and backcrossed into the respective deletion mutants to confirm the functionality of the fusion proteins. GCK1-GFP displays a localization at forming septa (Figure 27A) and the GFP-tagged NDK1 shows no significant localization besides an overall distribution in the cytoplasm (Figure 27B).
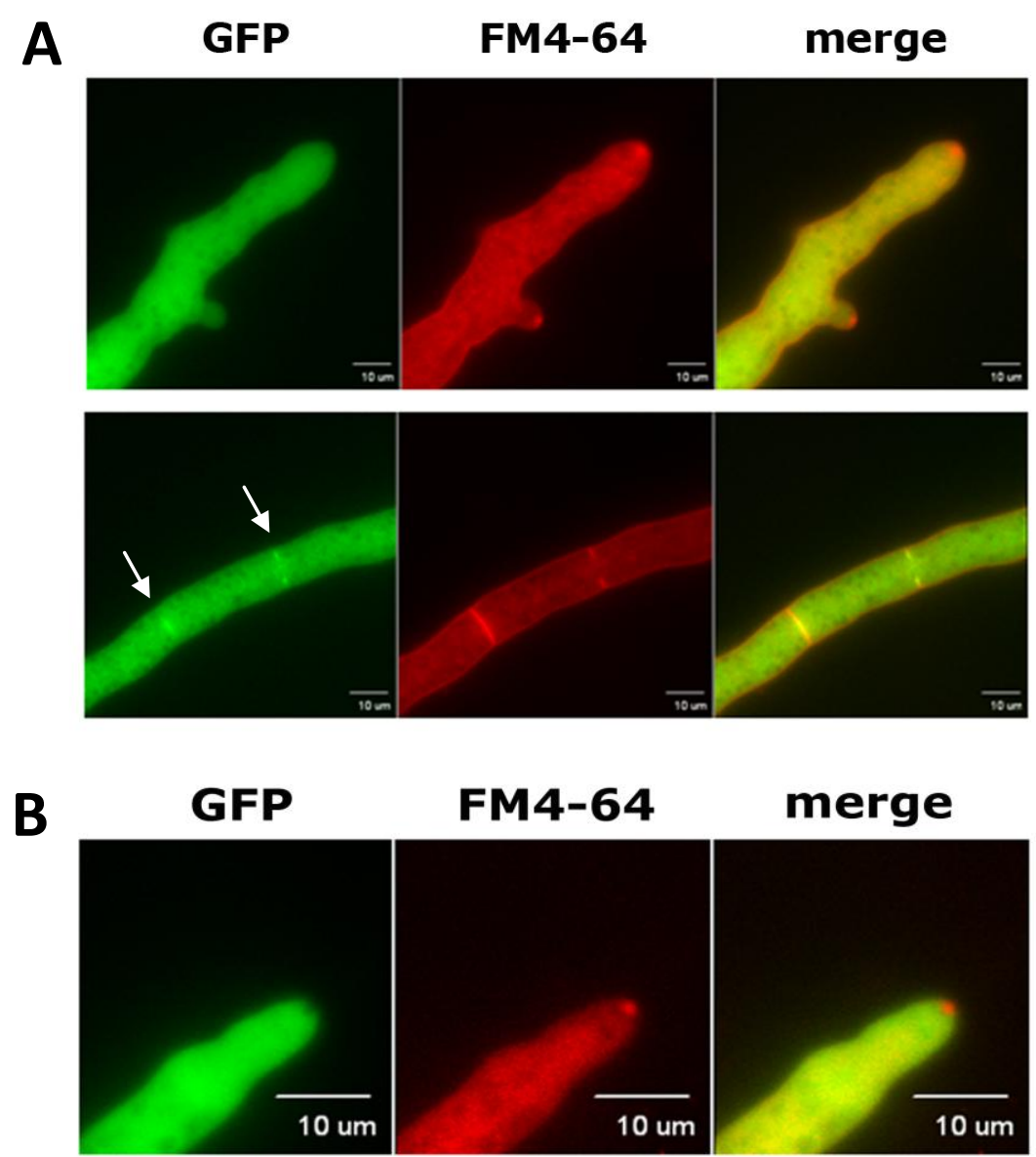

FM4-64 merge

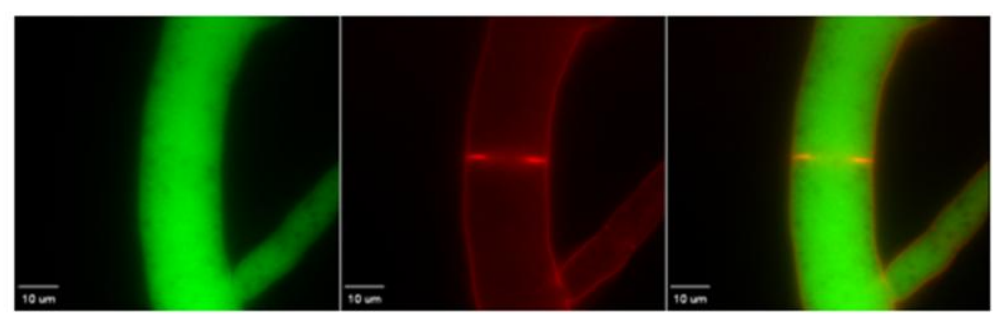

Figure 27: Localization of GFP-tagged GCK1 and NDK1.

(A) gck1::gfp localizes at forming septa (marked by arrows) and throughout the cytoplasm.

(B) The GFP-tagged NDK1 localizes throughout the cytoplasm, but shows no other significant localization pattern. Plasma membrane and the Spitzenkörper were stained with FM4-64 (mRFP filter). Scale bars are $10 \mu \mathrm{m}$. 
Furthermore, a dominant-negative form of $n d k-1$ was cloned by introducing a point mutation which leads to an aa substitution from cysteine 116 to tyrosine. This residue locates next to the putative active center histidine 117 (Yamamoto et al., 1995). The mutation of $n d k-1$ resulted in greatly reduced kinase activity compared to a wild type $n d k-1$ gene product, demonstrated by a kinase activity assay (data not shown). The DN $n d k-1$ strain shows a normal growth rate and hyphal morphology similar to the wild type, but a shortened aerial mycelium. The strain is also unable to form protoperithecia and to undergo cell fusion (Figure 28A-B). The MAK2 activity is decreased under normal growth conditions in contrast to the wild type and no longer inducible under oxidative stress (Figure 28C). Summarizing, only certain functions important for sexual development are disrupted in a DN $n d k-1$ strain in contrast to the $n d k-1$ deletion mutant. 
A
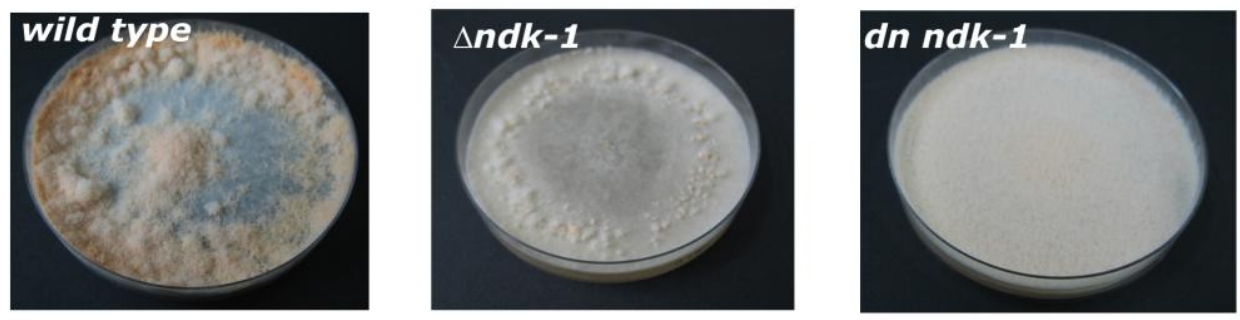

B

\begin{tabular}{|c|c|c|c|}
\hline & wild type & $\Delta n d k-1$ & $d n$ ndk-1 \\
\hline $\begin{array}{l}\text { Growth rate } \\
\text { (cm/day) }\end{array}$ & $3,9( \pm 0,2)$ & $1,2( \pm 0,2)$ & $3,8( \pm 0,2)$ \\
\hline Hyphal morphology & wild type & knobby & wild type \\
\hline $\begin{array}{l}\text { Vegetative cell } \\
\text { fusion }\end{array}$ & yes & yes & no \\
\hline $\begin{array}{l}\text { Arial mycelium and } \\
\text { conidiation }\end{array}$ & wild type & $\begin{array}{l}\text { poor and } \\
\text { clumped }\end{array}$ & shortened \\
\hline $\begin{array}{l}\text { Protoperithecia } \\
\text { formation }\end{array}$ & yes & yes & no \\
\hline
\end{tabular}

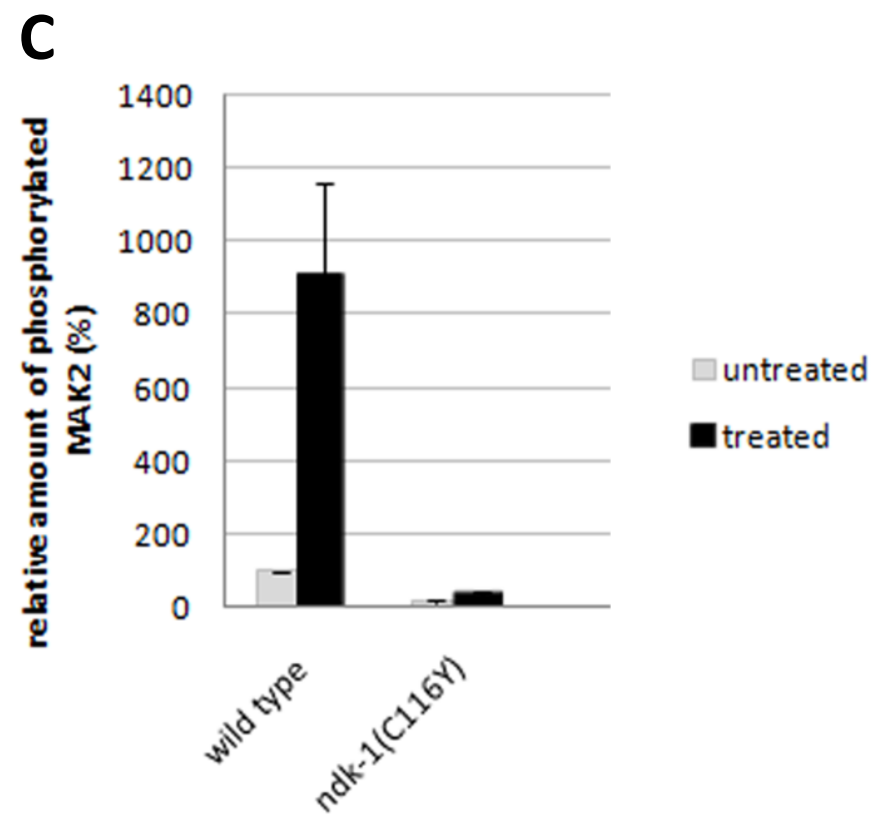

Figure 28: Phenotypic characterization of DN $n d k-1$ in contrast to wild type and $\Delta n d k-1$.

(A) Colony morphology of the indicated strains grown at $25^{\circ} \mathrm{C}$ on minimal media plates is shown.

(B) Overview about the phenotypic characteristics of the DN ndk-1 strain compared to the wild type and ndk-1 deletion mutant.

(C) Measurement of the MAK2 activity which is increased under normal and stress-induced growth conditions. 


\subsection{Regulation of the MAK2 pathway by the Striatin complex}

Summarizing, HYM1 displays developmental defects similar to MAK2 mutants and is important for MAK2 activity. Further, HYM1 interacts with NDK1 and GCK1 and both proteins also interact with proteins of the MAK2 cascade, but are not important for MAK2 activity and exhibit a different deletion phenotype compared to hym-1 and mak-2 mutants.

A glance into the literature indicated that NDK1, GCK1 and MAP kinases occured together in the striatin complex which play important roles in endocytosis, signal transduction, cell adhesion and virus assembling (Benoist et al., 2006).

Striatin acts as a scaffolding protein which forms a propeller-like structure for various protein-protein interactions in different organisms. One of the first striatin interacting proteins identified by yeast two-hybrid screens from Rattus norvegicus was the phocein/MOB3 (Baillat et al., 2001). Striatin and phocein mutants show also developmental and cell fusion defects like $\Delta$ hym-1 or MAK2 kinase cascade mutants (Benoist et al., 2006, Maerz et al., 2009, Maerz et al., 2008). We got yeast two-hybrid constructs of Pro11 (striatin homologue) and Mob3 of Sordaria macrospora from Yasmine Bernhards of the S. Pöggeler group and detected interactions of Pro11(Sm) and Mob3(Sm) with HYM1, NDK1 and proteins of the MAK2 kinase cascade (data not shown). To verify these results by co-IPs in N. crassa, epitope-tagged HAM3 (striatin homologue in N. crassa), MOB3, GCK1, NDK1, NRC1 and HYM1 were expressed in a nic-3 or trp-1 strain. The forced heterokaryons which were able to grow on minimal medium by complementation of the individual auxotrophies were used. Interactions of HAM3 and HYM1, NDK1, GCK1 and NRC1 were detected (Figure 29, Supplementary Figure 2). 


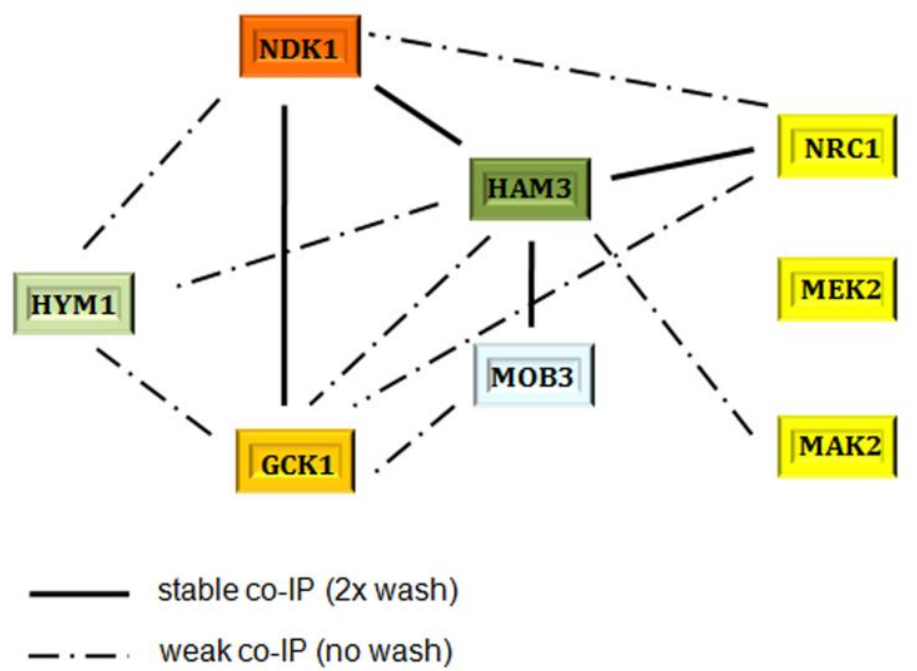

Figure 29: Summary of the interactions between HAM3, MOB3, HYM1, NDK1, GCK1 and proteins of the MAK2 cascade verified by co-immunoprecipitation experiments.

Due to the strong interaction between HAM3 and NRC1, both dominant-active NRC1 constructs were transformed into the $\Delta$ ham-3 mutant. Surprisingly, the point-mutated dominant-active construct rescued the $\Delta h a m-3$ deletion phenotype. $\Delta h a m-3$ with the constitutive active NRC1 was able to grow with normal aerial mycelium, to form protoperithecia and to perform hyphal cell fusion in contrast to $\Delta h a m-3$ with growth and developmental defects (Table 7, Supplementary Figure 3).

Table 7: The point-mutated DA NRC1 construct rescued the $\Delta$ ham-3 deletion phenotype.

\begin{tabular}{|c|c|c|c|c|}
\hline & $\Delta h a m-3$ & flag-nrc-1:: $\Delta$ ham-3 & $\begin{array}{c}\text { flag-nrc-1 }{ }_{3}^{\text {P448s }}:: \Delta h a m- \\
\end{array}$ & flag-nrc-1 ${ }^{\text {deletion ac41-590 }:: \Delta h a m-3}$ \\
\hline Growth rate & $1,9( \pm 0,3)$ & $1,7( \pm 0,1)$ & $2,9( \pm 0,3)$ & $1,8( \pm 0,3)$ \\
\hline $\begin{array}{l}\text { Aerial } \\
\text { mycelium }\end{array}$ & no & no & yes & no \\
\hline PP formation & no & no & yes & no \\
\hline $\begin{array}{l}\text { Germling } \\
\text { fusion }\end{array}$ & no & no & yes & no \\
\hline
\end{tabular}

In the earlier sections, the different phenotypes of HYM1, NDK1, GCK1 and the MAK2 mutants were described. In contrast to the mentioned mutants, $\Delta h a m-3$ and $\Delta m o b-3$ displayed diverse phenotypical characteristics. The ham-3 deletion mutant showed a wild type hyphal morphology with reduced aerial mycelium and a slow growth rate. The strain is not able to perform vegetative cell fusion and to form protoperithecia. The MAK2 activity is slightly increased under normal and stress-induced growth conditions. The mob-3 deletion 
mutant showed also a wild type hyphal morphology and a normal growth rate in contrast to the ham-3 deletion mutant. Moreover, $\Delta m o b-3$ displayed the same developmental defects as observed for $\Delta$ ham-3 (Figure 30). In summary, the deletion strains of HYM1, proteins of the MAK2 cascade, HAM3 and MOB3 display identical developmental defects but different morphological characteristics.
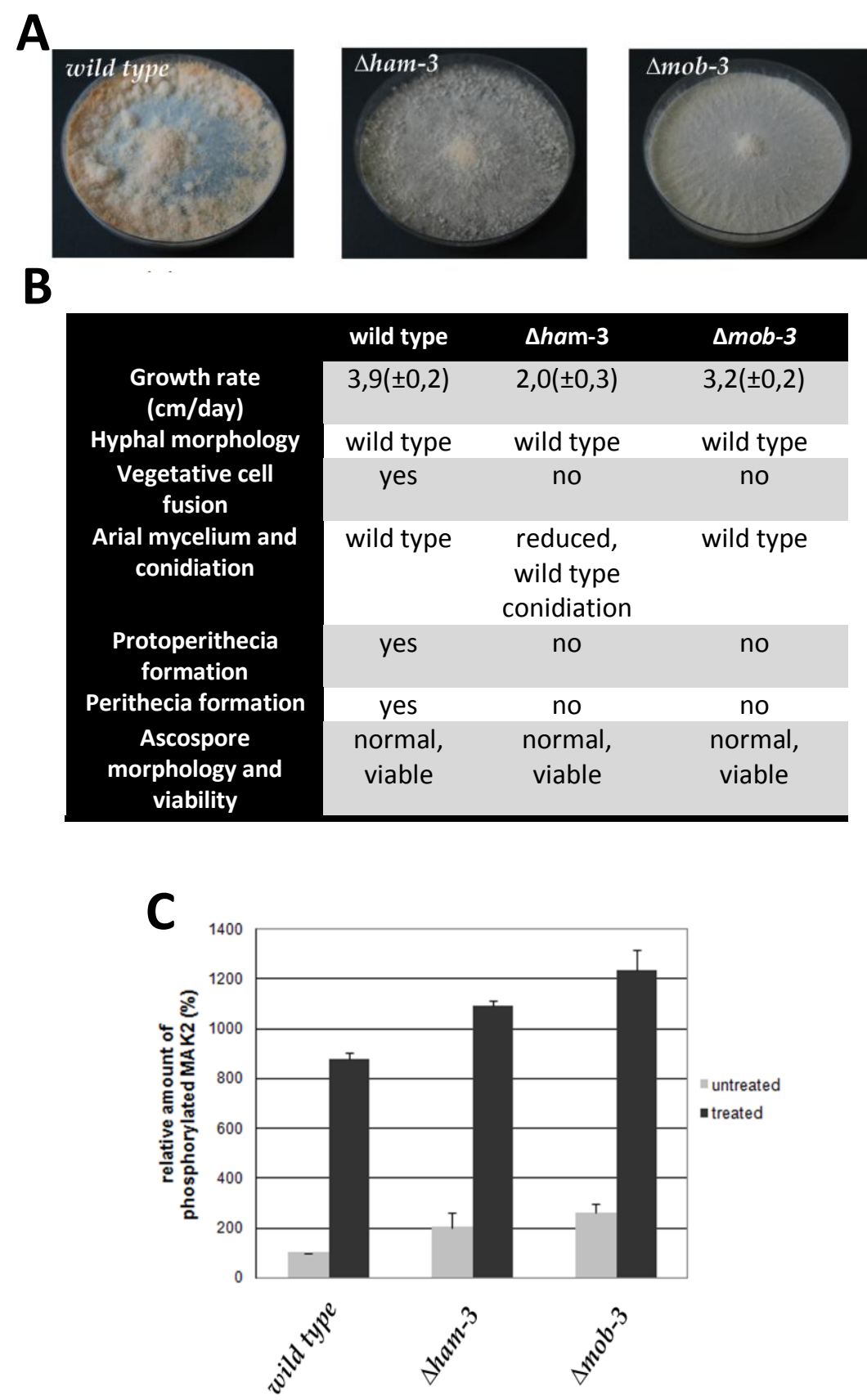

Figure 30: Phenotypic characterization of ham-3 and mob-3 deletion mutants.

(A) Colony morphology of the indicated strains grown at $25^{\circ} \mathrm{C}$ on minimal media plates is shown.

(B) Overview about the phenotypic characteristics of both mutant strains compared to the wild type.

(C) Measurement of the MAK2 activity which is increased under normal and stress-induced growth conditions. 
Furthermore, the localization pattern of HAM3 and MOB3 was determined. Therefore Cterminally GFP-tagged constructs were generated and backcrossed into the respective deletion mutants. For HAM3-GFP a localization as circular structures in the cytoplasm could be observed, which may represent the nuclear envelopes (Figure 31A). The same localization pattern could be identified for GFP-tagged MOB3 (Figure 31B).
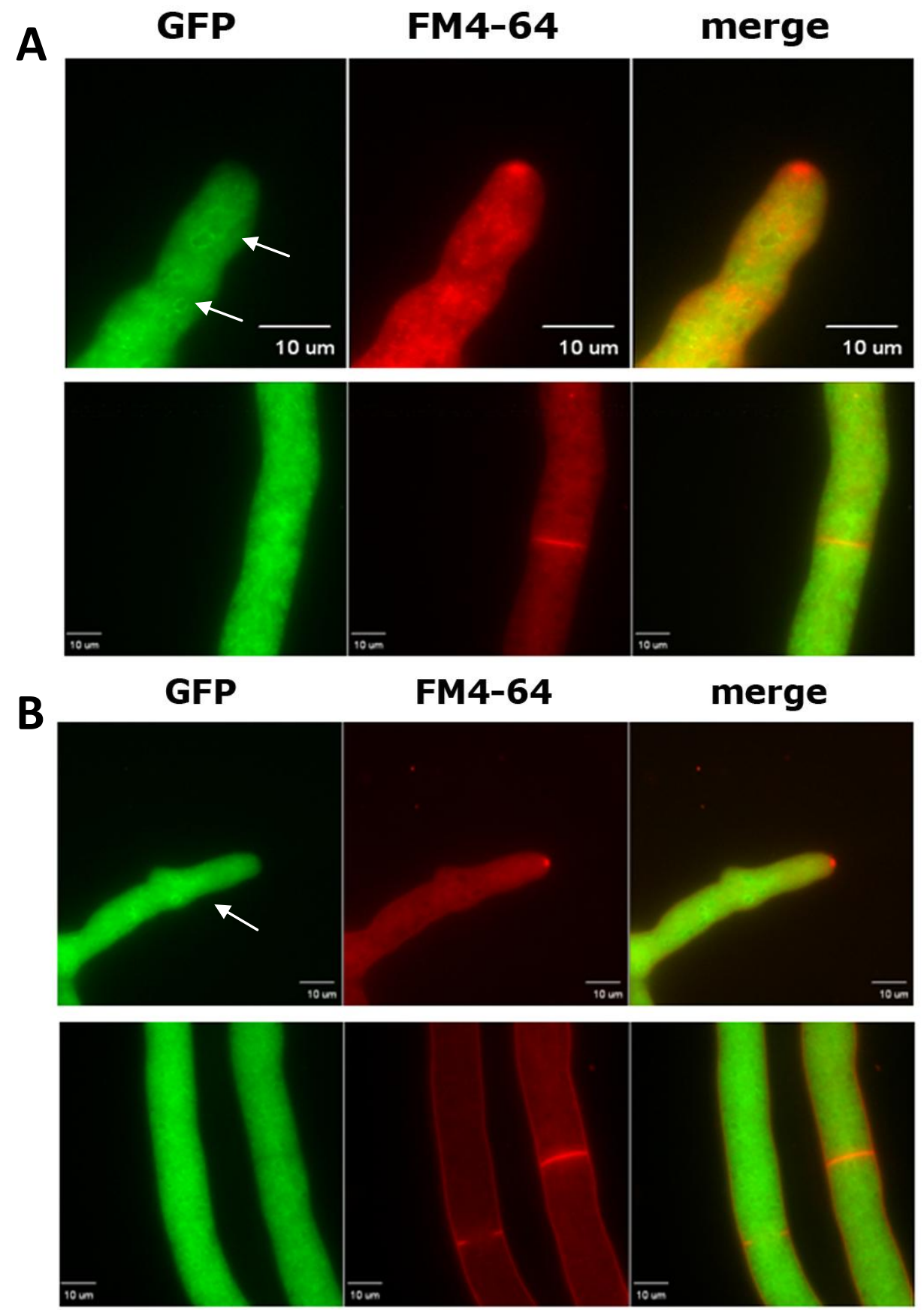

Figure 31: Localization pattern of HAM3-GFP and MOB3-GFP.

(A) GFP-tagged HAM3 localizes as circular structures in the cytoplasm marked by arrows and also throughout the cytoplasm.

(B) The construct mob3::gfp shows the same localization compared to GFP-tagged HAM3 indicating the presence of both proteins in one complex (arrows marked the nuclear envelopes). Plasma membrane and the Spitzenkörper were stained with FM4-64. Scale bars are $10 \mu \mathrm{m}$. 
Co-localization studies with GFP-tagged HAM3 or GFP-tagged MOB3 and RFP-tagged histone H1, which stains nuclei red, were performed to identify the circular structures in the cytoplasm. The strains were fused on a minimal medium plate and in some parts of the resulting non-forced heterokaryon a localization of HAM3 at the nuclear envelope was observed (Figure 32).

ham3

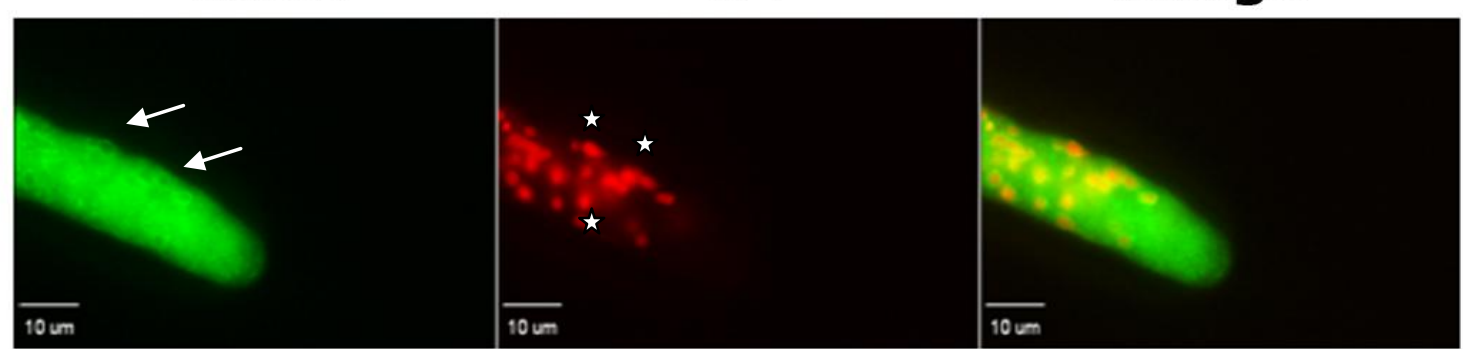

Figure 32: Co-localization GFP-tagged HAM3 (marked by arrows) and RFP-tagged H1 (marked by asterisks). HAM3-GFP localizes at the nuclear envelopes.

Additionally to the co-localization studies with RFP-tagged histone $\mathrm{H} 1$, BiFC constructs of HAM3 and MOB3 are still in progress for direct co-localization analysis. 


\section{Discussion}

\subsection{COT1 dimerization and interaction with MOB requires overlapping regions}

In this study, the detailed analysis of the NDR kinase COT1 and its regulation through the upstream GC kinase POD6 and the two adaptor MOB2 proteins in the filamentous ascomycete $N$. crassa was performed. An advantage of fungal models is their genetic tractability that allows quantitative analysis of in vitro and in vivo characteristics of proteins under endogenous expression conditions. In contrast to that, most data obtained in animals are derived from in transient overexpression experiments.

NDR kinases interact with specific adaptor proteins called MOBs. The NDR kinase COT1 in the filamentous fungus $N$. crassa is regulated by the combined function of MOB2A and MOB2B. This is evidenced by the co-purification of COT1 with these two MOBs, the cot-like synthetic phenotype of a $\Delta m o b-2 a ; \Delta m o b-2 b$ double mutant and the reduced in vitro kinase activity of COT1 in that double mutant (Maerz et al., 2009).

The cot-1 locus allows the translation of two isoforms. The length of the N-terminus of the short isoform corresponds to the $\mathrm{N}$-terminus of animal NDR kinases, while the long version contains a fungal-specific extension of 118 amino acids. The sequence of the $\mathrm{N}$-terminal extension is poorly conserved, but its presence is a feature of most fungal NDR kinases and characterized by a high abundance of the amino acids asparagine and glutamine (e.g. $24 \%$ and $36 \%$ asparagine and glutamine residues in N. crassa COT1 and S. cerevisiae Cbk1p, respectively).

By yeast two-hybrid analysis, it was demonstrated that the region directly preceding the kinase domain (amino acids 119-212 of COT1(long)) is sufficient for homodimerization of COT1 and homodimerization has been shown for several other NDR kinases (Hou et al., 2004, Nelson et al., 2003). Additionally, this N-terminal expansion is required for the formation of СОТ1-МОВ2 heterodimers and further involved in the stimulation of the kinase activity. The signal that regulates the transition from the inactive COT1 homodimer to the basal active СОТ1-МОВ2 heterodimer is currently unknown. The level of MOB binding to COT1(long) and COT1(short) in vivo is similar, but the COT1 kinase activities in vitro are different, indicating that kinase activation is not only dependent on MOB binding, but 
requires an additional level of regulation. This may include additional interacting proteins and/or posttranslational modifications of the kinase. The reduced stability of COT1(short) homodimers may be a reason for the degradation of COT1(short) in the mob-2 double deletion background. This suggestion is consistent with data from budding and fission yeast. The size of ORB6 corresponds to the short COT1 isoform, and shutoff experiments of mob-2 in S. pombe result in degradation of ORB6. Budding yeast Cbk1p has a long N-terminal extension and deletion of mob-2 does not affect Cbk1p stability in S. cerevisiae (Hergovich et al., 2006b, Jansen et al., 2006). The presence of a second ATG and thus the potential for the presence of two expressed isoforms in $N$. crassa and related species (e.g. Podospora anserina, Magnaporthe grisea and Sclerotinia sclerotiorum) allows the prediction that the stability of animal NDR kinases is also regulated through their interaction with MOB proteins. However, no data are currently available regarding animal knock down experiments of MOB proteins and their impact on endogenous NDR levels.

In conclusion the fungal specific $\mathrm{N}$-terminal expansion is responsible for the interaction of СОT1 with itself or МOB2 proteins, suggesting the presence of СОT1 homo- or СОT1-МOB2 heterodimers in the cell. This could be a possible mechanism of regulating NDR function by dimer formation (Figure 33). 


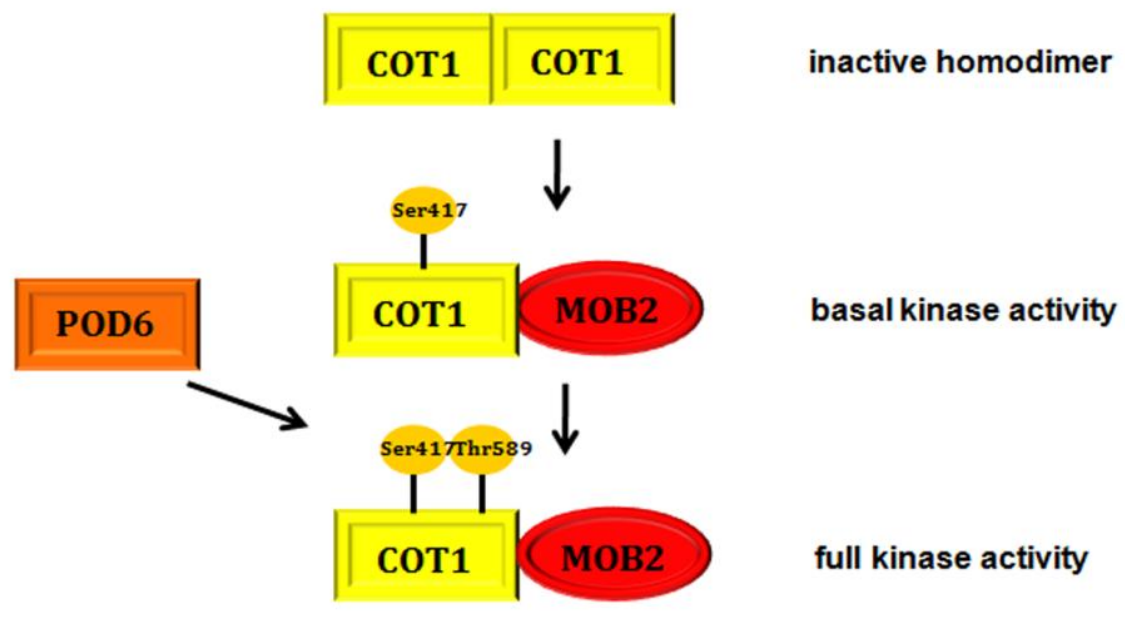

Figure 33: Model of the potential regulation of COT1 by dimer formation.

Inactive COT1 is forming a homodimer. The COT-MOB heterodimer may be the first step in activation of the kinase and is regulated via competing interaction sites in the $\mathrm{N}$ - terminus of COT1 (Maerz et al., 2009),

COT1-MOB2 heterodimerization and Ser417 autophosphorylation relieve the autoinhibition of the COT1 homodimer and result in basal activity of the СОТ-МOB complex. The subsequent phosphorylation of Thr589 through POD6 leads to a conformational change and full activation of the NDR kinase.

\subsection{Threonine phosphorylation of the NDR kinase COT1 by the upstream kinase POD6 is necessary for kinase activity and localization}

Artificial membrane targeting of animal NDR kinases or of MOB1 adaptors increases NDR activity (Hergovich et al., 2005, Ho et al., 2010). So far, the mechanism of kinase transfer to the membrane is still unresolved. In the filamentous fungus the phosphorylation of the threonine residue 589 by POD 6 controls not only the in vitro activity of COT1, but also the membrane association of the COT1-MOB complex.

Ectopically expressed COT1-GFP complemented the defects of the $\Delta \cot -1$ deletion mutant and localized as a faint cap structure in growing hyphal tips and further co-localized with a vesicle accumulation in the subapical region, called the Spitzenkörper. In contrast, COT1(T589A)-GFP did only poorly complement the cot-1 deletion and resulted in a strain with morphology and growth rate comparable to the myc-cot-1(T589A) strain (data not shown).

By ectopically expression of COT1(T589A)-GFP in a wild type background, only an accumulation at the Spitzenkörper could be observed, but no localization at a faint-cap like structure at the hyphal apex, indicating that hydrophobic motif phosphorylation is required 
for membrane targeting and functionality of COT1. This coupling of activation and localization by a single phosphorylation event seems to be the primary reason for the poor functionality of COT1(T589A) in vivo, because T2A has activity high enough to facilitate modest growth (Maerz et al., 2009).

In a next step, artificially membrane-targeted СОT1 variants were generated by attaching the consensus motif for $\mathrm{N}$-terminal palmitylation/myristylation (pm; M-G-C-x-x-S-A/S/T) to the N-terminus of COT1. Ectopically expressed pm-COT1-GFP associated with the plasma membrane along the whole hypha in a patchy manner, confirming the suitability of the tag. In addition, abundant cytosolic spots were detected that may constitute COT1 associated with vesicular transport intermediates.

In contrast to the partial suppression of $\Delta$ pod-6 obtained by myc-COT1(T589E), pm-mycCOT1(T589E) fully suppressed the $\Delta$ pod-6 phenotype, suggesting that Thr589 phosphorylation of COT1 by POD6 is an important event for membrane association of COT1 and is required for its localization to the hyphal apex during polar growth. Surprisingly, pmmyc-COT1 and pm-myc-COT1(T589A) also fully suppressed the $\Delta p o d-6$ defects indicating an independence of phosphorylation by POD6, when COT1 is artificially targeted to the plasma membrane.

In conclusion, artificial targeting of the COT1 to the plasma membrane in cells lacking the endogenous targeting signal (phosphorylation of Thr589 by POD6) is more important for the in vivo functionality of the NDR kinase than its Thr589 phosphorylation-dependent maximal kinase activity. Moreover the measured kinase activities of the pm-COT1 variants exhibited decrease in activity up to $50-65 \%$ relative to the non-targeted controls (Maerz and Seiler, unpublished data). Thus, altered kinase activity cannot account for the suppressive effect of the palmityl-myristyl-tagged COT1 versions in $\Delta p o d-6$.

In a further experiment, the suppression of $\Delta p o d-6$ by targeting MOB2A (and thus presumable the COT1-MOB2 complex) to the membrane using a pm-HA-mob-2a construct in a $\Delta$ pod-6 background was detected. A full suppression by the pm-MOB2A could also be observed, indicating that POD6 is no more needed for COT1 phosphorylation if the COT1 adaptor protein MOB2 is directly localized to the plasma membrane.,

In a next step, it will be tested if pm-COT1(T589E) is also able to rescue the $\Delta m o b 2 a$; $\Delta$ mob2 $b$ double mutant. If $\mathrm{pm}$-СOT1(T589E) is not able to rescue the $\Delta$ mob2a; $\Delta$ mob2b deletion, one could assume that both the interaction with the adaptor protein MOB2 and 
the Thr589 phosphorylation by POD6 are essential for the correct localization of COT1 at the hyphal tip.

\subsection{HYM1 acts as a dual scaffold for the COT1 complex and the MAK2 cascade}

The potential scaffolding protein HYM1 has been reported to interact with NDR kinases in several organisms (Kume et al., 2007). To determine the role of HYM1 in N. crassa, yeast two-hybrid assays between the NDR network components COT1 and POD6 were performed. Positive interactions were detected for COT1-HYM1 and POD6-HYM1 suggesting the formation of a complex established by these three proteins. To confirm these interactions an immunoprecipitation experiment with myc-tagged HYM1 was accomplished which recovered both COT1 and POD6. A stable in vivo interaction of COT1 and POD6 could be strengthened by the use of washing buffer containing $0.5 \mathrm{M} \mathrm{NaCl}$.

In addition, a strain containing HA-tagged POD6 and myc-tagged COT1 was generated and crossed into the hym-1 deletion background for a co-immunoprecipitation experiment. The interaction between COT1 and POD6 is reduced in $\Delta h y m-1$, suggesting HYM1 as a linker protein between the two kinases COT1 and POD6. The assumption that HYM1 and COT1 acting in the same complex is also confirmed by the partial overlapping localization of COT1 and HYM1 GFP-tagged constructs at septa and in the putative Spitzenkörper at the hyphal apex.

Mutations in cot-1, pod- 6 and mob-2 genes as the central components of COT1 signaling pathway share characteristic defects, such as cessation of hyphal elongation with a needleshaped apex and the generation of massive amounts of extension-arrested new tips along the entire cell (Maerz et al., 2009, Seiler et al., 2006, Yarden et al., 1992). Surprisingly, deletion of hym-1 did not develop this "barbed wired" phenotype, but generated characteristic developmental defects. The morphological characteristics of $\Delta h y m-1$ are identical to the defects caused by mutations in each of the three kinase genes of the MAK2 MAP kinase pathway (stunted aerial mycelium, derepressed conidiation, ascospore lethality and the inability to develop protoperithecia) (Li et al., 2005, Maerz et al., 2008, Pandey et al., 2004). Due to similar phenotypes of hym-1 and mak-2 mutants we assume a connection of the COT1 signaling pathway and the MAK2 cascade via HYM1. This assumption is strengthened by the fact that mak-2 pathway mutants were identified as partial suppressors of the cot-1 (ts) growth defect at restrictive conditions. Furthermore, $\Delta m a k-2$ cell fusion and 
developmental defects were overcome in a cot-1 (ts) background at permissive conditions, again indicating a connection between both pathways (Maerz et al., 2008).

The finding that the MAK2 activity is abolished in hym-1 deletion background indicated that HYM1 is essential for the signal transduction in the MAP kinase cascade. To further determine if and how HYM1 interacts with the components of the MAK2 pathway, different approaches were performed. Co-immunoprecipitation experiments of tagged proteins in $N$. crassa exhibited weak interactions between HYM1 and the three components of the MAK2 cascade. Surprisingly, interactions between these proteins could not be observed by yeast two-hybrid studies, suggesting that interactions between HYM1 and proteins of the MAK2 pathway may be not direct. So, one hypothesis is that the indirect linker HYM1 acts together with a so far unknown protein providing a direct scaffold for the MAP kinase cascade. The protein of unknown function could also be a scaffold protein and together with HYM1 it may form a platform for the MAP kinase cascade. In this case, HYM1 together with the unknown protein would adopt the role of Ste5p in Saccharomyces cerevisiae which brings the kinases of the MAP kinase cascade in a close proximity (Seeliger \& Kuriyan, 2009).

To further elucidate the hierarchical order of HYM1 and the MAK2 pathway kinases, dominant-active constructs of the MAK2 MAP kinase kinase kinase NRC1 were generated and introduced in $\Delta h y m-1$. Strikingly, these constructs suppressed only the growth rate of $\Delta h y m-1$, but not the loss of MAK2 activity. This finding on the one hand implies that the dominant-active NRC1 is not sufficient for a functional MAK2 cascade and on the other hand it indicates that HYM1 does not act upstream of the triple MAP kinase. Unfortunatly, we cannot exclude that the dominant-active constructs may not function constitutively due to the fact that we do not know which protein acts upstream of the MAK2 kinase cascade and therefore can not test the activity status of DA NRC1. A similar approach, which is still in progress is the generation of a dominant-active construct of the double MAP kinase MEK2. In this case we will be able to confirm the expression of the constitutively active MEK2 by transforming this construct into the deletion mutant of the triple MAP kinase NRC1. Thus, DA MEK2 should full complement the $\Delta n r c-1$ mutant.

So far, we conclude that HYM1 is a bona fide component of the COT1 complex and necessary for the interaction between COT1 and POD6. Second, based on the loss of MAK2 activity and the phenotypical characterization, HYM1 is important for the signal transduction within the MAP kinase cascade. 
In future experiments, the question whether HYM1 is not only used as a scaffold in the COT1-POD6 complex, but may also acts as an indirect linker of the MAK2 cascade.

\subsection{HYM1 functions as a master regulator of Ste20 kinases}

Mo25 proteins have evolved as key regulators of Ste20 kinases and may represent a core mechanism of regulating conformation of pseudokinases which activating catalytically competent protein kinases. In our first studies we identified HYM1 as a scaffold protein of the NDR kinase COT1 and the Ste20 kinase POD6 which play a critical role in polar growth. In addition, we could show that HYM1 is necessary for the signal transduction in the MAK2 MAP kinase cascade, but is not the direct linker of the MAP kinases.

In mammals MO25 operates as a scaffolding subunit required for the activation and stabilization of the LKB1 tumour suppressor protein kinase complex which functions as the master upstream regulator of AMP protein kinases (Boudeau et al., 2003a, Boudeau et al., 2003b, Boudeau et al., 2003c, Boudeau et al., 2004). The LKB1 protein kinase occurs as a heterotrimeric protein consisting of MO25 complexed to LKB1 and the catalytically inactive pseudokinase STRAD (Baas et al., 2003, Boudeau et al., 2003a, Boudeau et al., 2003b, Boudeau et al., 2006, Boudeau et al., 2003c, Boudeau et al., 2004). Furthermore, MO25 isoforms interact in a similar manner with five other Ste20 kinases, namely SPAK, OSR1 (regulators for homeostasis and blood pressure) and MST3, MST4, YSK1 (regulators of morphogenesis and polarity) in mammals (Filippi et al., 2011). These data correlate with our findings in Neurospora crassa. As I mentioned before, HYM1 interact with POD6, a STE20 kinase, which plays a critical role in determing polarity. On the other hand we discovered that HYM1 interacts with proteins of the MAK2 kinase cascade which is important for sexual development and cell fusion.

By performing a yeast two-hybrid screen two additional, potential interaction partners of HYM1 were identified, a germinal centre kinase GCK1 and a nucleoside-diphosphate kinase NDK1. Thus, co-immunoprecipitation experiments in the fungus were performed to verify the results of the yeast two-hybrid approach. Additionally, interactions between GCK1 and NDK1 with the MAK2 kinase cascade were determined. Due to the fact that it was not possible to verify direct interactions between HYM1 and proteins of the MAK2 kinase cascade by yeast two-hybrid studies, we hypothesized that maybe GCK1 and NDK1 act as direct linker proteins between HYM1 and the MAK2 pathway. The comparison of the MAK2 
activity and morphological characteristics like the ability to form sexual structures and hyphal cell fusion between the deletion mutants hym-1, gck-1, ndk-1 and mak-2, revealed distinct phenotypes for $\Delta g c k-1$ and $\Delta n d k-1$. However, a similar phenotype of $\Delta h y m-1$ and $\Delta m a k-2$ could be observed. These results neutralized our hypothesis that GCK1 and NDK1 act as direct linkers between HYM1 and the MAK2 pathway but may act as modulators.

In yeast two-hybrid experiments, we identified an additional interaction between HYM1 and the PAK kinase STE20, and this interaction has to be verified in future experiments. Thus, HYM1 interacts with different Ste20 kinases (POD6, GCK1, STE20) in Neurospora crassa. These findings correlate with the published data of Filippi et al., 2011 in which MO25 was determined as a master regulator of SPAK/OSR1 and MST3/MST4/YSK1 protein kinases which belong to the Ste20 kinases. So it is possible that HYM1 interacts with different Ste20 kinases due to different upstream signals like pheromones, and the respective kinase-HYM1 complex activates distinct downstream pathways, such as MAK2 MAP kinase cascade or the COT1 pathway. The identification of the downstream proteins, the upstream input signals and the hierarchical order of HYM1, proteins of the MAK2 cascade and the Ste20 kinases will be the purpose of future investigation.

The second identified HYM1 interacting protein NDK1 belongs to the group of nucleosidediphosphate kinases (NDKs) which catalyze the transfer of the terminal phosphate from NTPs to NDPs, thereby maintaining the nucleotide metabolism and also function as an ubiquitous enzyme in prokaryotes and eukaryotes (Kyriakis, 1996). Further NDKs also play a role during several signal transduction pathways by forming oligomers and interaction with other proteins (Amendola et al., 2001, Lee \& Lee, 1999, Lee et al., 1997).

NDK null mutants of budding and fission yeast show normal vegetative growth, sporulation, mating and morphology though they display a much lower NDK activity in contrast to the wild type (Fukuchi et al., 1993, Izumiya \& Yamamoto, 1995). In plants, NDK interacts with phytochromes, photoreceptors that relay environmental light signals (Choi et al., 1999, Hasunuma \& Yabe, 1998). Higher organisms contain multiple isoforms of NDK with tissuespecific expression. In Drosophila melanogaster, Caenorhabditis elegans and Xenopus laevis NDKs are essential for development (Chiadmi et al., 1993, Maeda et al., 2001, Ouatas et al., 1997, Ouatas et al., 1998). In mammals, NDKs are involved in differentiation, cell survival 
and proliferation (Lee \& Lee, 1999, Lee et al., 1997, Postel et al., 2000a, Postel et al., 2000b, Roymans et al., 2001, Roymans et al., 2000).

Not much is known about NDK in filamentous fungi, only Lee et al., 2006 reported that mutated NDK displayed reduced NDK activity and deficient light response for perithecial polarity. In contrast to the wild type, the deletion mutant ndk-1 in N. crassa exhibits growth defects and the MAK2 activity is reduced indicating that NDK1 is important for MAK2 activity. Furthermore, the sexual development as well as cell-cell fusion is not affected in the deletion mutant which stands in contrast to a $D N n d k-1$ version. The downregulation of NDK1 causes a defect in protoperithecia formation, hyphal fusion and a reduced MAK2 activity. Moreover, $D N n d k-1$ displays a phenotype similar to mak-2 and hym-1 deletion mutants suggesting a role for NDK1 in the HYM1-MAK2 pathway. Due to the fact that NDKs in higher eukaryotes play important roles in development and differentiation, the interaction between NDK1, HYM1 and the MAK2 kinase cascade is not devious.

\subsection{HAM3, the $N$. crassa striatin homologue negatively regulates the MAK2 pathway}

Proteins of the striatin family are highly conserved from eukaryotes to mammals with the exception of budding yeast and plants (Benoist et al., 2006). These proteins are multidomain molecules containing several protein-interacting motifs for establishing various proteinprotein interactions.

In humans, striatin is required for the polarized development of dendritic spines(Bartoli et al., 1998). Polarized development is also a defining trait of the filamentous fungal lifestyle.

In $F$. verticilloides $\Delta f s r 1$ a reduced mycelia radial growth was observed while an increased density of aerial mycelium was produced in S. macrospora pro11 mutant (Poeggeler \& Kueck, 2004, Shim et al., 2006). In N. crassa ham-3 mutant causes growth and conidiation defects. All these observations suggest that disruption of striatin orthologs affects fungal growth at the colony forming level, but not hyphal polarity (Wang et al., 2010).

The Aspergillus strA deletion mutant produced smaller cleistothecia with ascospores whereas the $F$. verticilloides fsr 1 mutant and the $N$. crassa ham-3 mutant fail to form protoperithecia, however still could fertilize protoperithecia (Shim et al., 2006). Also the S. macrospora pro11 mutant is arrested in sexual development at the stage of transition from protoperithecia to perithecia, formed fewer protoperithecia and lacked ascospores 
(Poeggeler \& Kueck, 2004). In conclusion, besides other striatin homologues also the $N$. crassa HAM3 is important for the formation of sexual structures.

The presence of a caveolin binding domain in striatin family proteins strongly suggests a function at membranes (Williams \& Lisanti, 2004a, Williams \& Lisanti, 2004b). Mammalian striatin family proteins were detected in cytosolic and membrane fractions and also the $S$. macrospora Pro11 was detected in a membrane fraction (Castets et al., 2000, Poeggeler \& Kueck, 2004). Wang et al. published in 2010 the localization of a StrA::eGFP construct to the ER and the nuclear envelope in Aspergillus nidulans. This correlates with the results of our GFP localization studies where we could observe a localization of HAM3 at the nuclear envelopes. Phocein, a protein known to interact with striatin in rat, localizes to ER and the Golgi apparatus in neurons and to the Golgi apparatus in HeLA cells (Baillat et al., 2001). In $N$. crassa, we observed the same localization pattern at the nuclear envelopes for MOB3 suggesting that HAM3 and MOB3 act in one complex. These data were confirmed by coimmunoprecipitation experiments in $N$. crassa, in which we could identify an interaction between both proteins. Furthermore, phocein/МОВ3 was the first identified striatin interacting protein (Baillat et al., 2001) and a comparison of both deletion phenotypes shows a lack of vegetative hyphal fusion, defects in growth, conidiation and sexual developmental defects (Simonin et al., 2010).

In addition, by a yeast two-hybrid screen it has been demonstrated that phocein associates with Esp15 and NDPK and partially co-localizes with Dynamin I in rats (Baillat et al., 2002, Baillat et al., 2001). Esp15 and NDPK serve as functional neighbours of Dynamin I, a GTPase which plays a critical role in endocytosis. Therefore, a role of striatin in endocytosis was suggested in animal cells and it could be possible that striatin also plays a role in endocytosis in fungi. We also identified a nucleoside-diphosphate kinase in $N$. crassa which interacts with HAM3, but so far we cannot integrate HAM3 and NDK1 in one complex in Neurospora.

In human endothelial cells, striatin is responsible for localizing the rapid activation of a MAP kinase (Lu et al., 2004). The MAP kinase MAK2 is required for vegetative cell fusion in $N$. crassa (Pandey et al., 2004). Recent work has shown that MAK-2 oscillates between the fusion tip and the cytoplasm in germlings undergoing chemotropic interactions (Fleissner et al., 2009a, Fleissner et al., 2009b). The mutants of the MAK2 kinase cascade show also developmental defects like observed for HAM3 and MOB3 deletion mutants in N. crassa. To discover a direct link between the HAM3-MOB3 complex and the MAK2 kinase cascade yeast 
two-hybrid studies and in vivo co-immunoprecipitation experiments were performed resulting in the determination of strong interactions between HAM3-MOB3 with NDK1 and NRC1, the triple MAP kinase of the MAK2 cascade (Supplementary Figure 2). The MAK2 activity is slightly increased in ham-3 and mob-3 deletions mutants, indicating a negative regulation of MAK2 by both proteins. To further analyze the hierarchical order of the HAM3MOB3 complex and the MAK2 kinase cascade, dominant-active constructs of the MAPKKK NRC1 were generated and crossed into the $\Delta h a m-3$ background. Surprisingly, the dominantactive constructs of NRC1 fully rescued the growth and the developmental defects of the deletion mutant (Supplementary Figure 3). Therefore we speculated that HAM3 acting upstream of the MAK2 cascade, but so far we do not know the exact function of HAM3 in this MAK2 pathway. Due to the fact that in higher eukaryotes striatin interacts with several MAP kinase pathways, it is not astonishing that a communication between the striatin homologue HAM3 and the MAK2 kinase pathway occurs in N. crassa (Benoist et al., 2006). The intent of this cross-communication has to be investigated in future experiments.

Furthermore, we identified a communication between the HAM3-MOB3 complex and the NDR COT1 signaling. In other experiments we determined that the NDR complex COT1-POD6 is indirectly linked to the MAK2 kinase cascade via HYM1. By co-immunoprecipitation experiments and performing an in vitro kinase activity assay, we first identified an interaction between HAM3 and COT1 and second that the HAM3 functions as a substrate of COT1. When the COT1-specific site in HAM3 is mutated to alanine or glutamate we do not observe a phosphorylation signal anymore, but we also do not observe any changes in fungal morphology or developmental growth behaviour (Supplementary Table 1, Supplementary Figure 4). In conclusion, we can establish a link between COT1 signaling, the MAK2 kinase cascade and the HAM3-MOB3 complex in N. crassa. This finding correlates with the publications of Kean et al., 2011 and Ribeiro et al., 2010 which indicate a negative regulation of the Hippo pathway by the STRIPAK complex in Drosophila melanogaster.

In future experiments, the generation of BiFC constructs of several components of the three interacting complexes (e.g. HYM1-COT1, СОT1-MAK2, MOB3-HAM3) would be interesting. This approach would help bringing the so far determined in vivo interactions in a spatial arrangement in the filamentous fungus $N$. crassa. Furthermore, the construction of different doubl- knockout strains of the HAM3-MOB3 complex and other proteins, which are part of 
this network (e.g. GCK1, NDK1) will be accomplished to determine a hierarchical order of the various components.

\subsection{Summarizing model of the cross-communication between NDR signaling, MAP kinase cascade and striatin complex that regulates sexual development and polar growth in $\mathbf{N}$. crassa}

During my thesis I was able to identify the cross-communication between the NDR COT1 kinase signaling, the MAK2 MAP kinase cascade and the striatin complex.

NDR signaling complexes play an important role in cell differentiation and polar morphogenesis in various organisms (Geng et al., 2000, Racki et al., 2000, Yarden et al., 1992, Zallen et al., 2000). In baker's yeast the NDR signaling complex of our interest consists of the NDR kinase Cbk1p, the MOB protein Mob2p and the upstream GC kinase termed Kic1p. Further two scaffolding proteins, Hym1p and Tao3p, were identified (Ho et al., 2002, Ito et al., 2001, Kurischko et al., 2005, Nelson et al., 2003). In S. pombe the NDR kinase Orb6, the kinase-associated protein Mob2, the upstream GC kinase Nak1 and also a Hym1p homologue Pmo25 were described (Hou et al., 2003, Kanai et al., 2005, Kume et al., 2007). In filamentous fungi and higher eukaryotes homologous proteins of the NDR kinase complex were also identified. In the filamentous ascomycete $N$. crassa, the NDR kinase COT1, the adaptor proteins MOB2A and MOB2B, the upstream GC kinase POD6 and the scaffolding protein HYM1 were identified as the core components of the NDR signaling complex important for polar growth (Seiler et al., 2006).

By yeast two-hybrid studies, different co-immunoprecipitation approaches and localization studies, interactions between these components were identified. In contrast to the "barbed wired" phenotype observed in cot-1 and pod-6 mutants the deletion mutant of hym-1 displays phenotypical differences and shares more similarities to the mutants of the MAK2 kinase cascade. Positive interactions between HYM1 and the proteins of the MAK2 kinase cascade were verified by various experiments. It was determined that the MAK2 activity but not the MAK1 activity is abolished in $\Delta h y m-1$ by performing a MAK2 activity assay. These data suggested that HYM1 is necessary for the signal transduction in the MAP kinase cascade und further indicated a direct communication between the MAK2 pathway and the NDR signaling complex. This cross-communication is strengthened by the facts that mak-2 mutants were identified as partial suppressors of the cot-1(ts) growth defect at restrictive conditions and that $\Delta m a k-2$ cell fusion and developmental defects were overcome in a cot-1 
(ts) background at permissive conditions (Maerz et al., 2008). In addition, recent studies described MO25, the HYM1 homologue in mammals as a master regulator of various Ste20 kinases (Filippi et al., 2011).

By approaches with dominant-active constructs of the triple MAP kinase NRC1 in $\Delta h y m-1$ I tried to clarify a hierarchical order between HYM1 and the MAK2 cascade. So far I could determine that the altered growth rate is rescued by the dominant-active NRC1 construct, but not the other phenotypical characteristics as well as the signal transduction in the MAP kinase cascade. This suggests that the link between HYM1 and the MAP kinase is first not direct and HYM1 does not function upstream of the MAK2 cascade. Therefore I prepared a yeast two hybrid screen of HYM1 to identify proteins which directly link HYM1 to MAP kinase proteins. Thereby a further Ste20 kinase GCK1 and a nucleoside-diphosphate kinase were identified as HYM1 and MAP kinase interaction partners. However, GCK1 and NDK1 acting not as direct linker proteins between HYM1 and the MAK2 kinase cascade verified by different morphological characterizations, localization studies and determining the MAK2 activity in the respective deletion mutants. In conclusion the protein(s), which directly link(s) HYM1 to the MAK2 kinase is (are) still lacking and whether HYM1 shuffles between the COT1-POD6 complex and the MAK2 MAP kinase cascade or if both complexes act in overlapping ways is still unresolved.

Additionally, a third complex including HAM3, a striatin homologue was identified to interact with components of the MAK2 kinase cascade and the NDR COT1 signaling complex.

Proteins of the striatin members play important roles in various pathways, and their misregulation causes developmental impairment. Here, I identified that HAM3 together with its interaction partner MOB3, interacts with proteins of the MAK2 kinase cascade. In addition, a dominant-active construct of the triple MAK2 MAP kinase NRC1 fully complemented the growth and developmental defects of the ham-3 deletion mutant suggesting that HAM3 acting upstream of the MAK2 pathway. Additionally, the MAK2 activity is increased in $\Delta h a m-3$ and $\Delta m o b-3$ and striatin functions as the $\mathrm{B}^{\prime \prime \prime}$ subunit of PP2A suggesting that PP2A negatively regulates MAK2 (Ribeiro et al., 2010).

So far, I could identify a complex network of proteins that is important for sexual development and polar growth. The proposed subcomplexes consist of various kinases and scaffolding proteins which display partial overlapping functions (Figure 34). The functional significance of the determined interactions between the described subcomplexes is still 
elusive and needs to be clarified in future experiments. The main interest should be to understand the relationship between the subcomplexes and their regulation. One approach may be the characterization of double deletion mutants to discover their hierarchical relationship. Furthermore, co-localization experiments by performing BiFC studies should be a point of investigation. Also the relevants of other proteins e.g. STE20, HAM2 and HAM4 in the context of the different networks should be clarified.

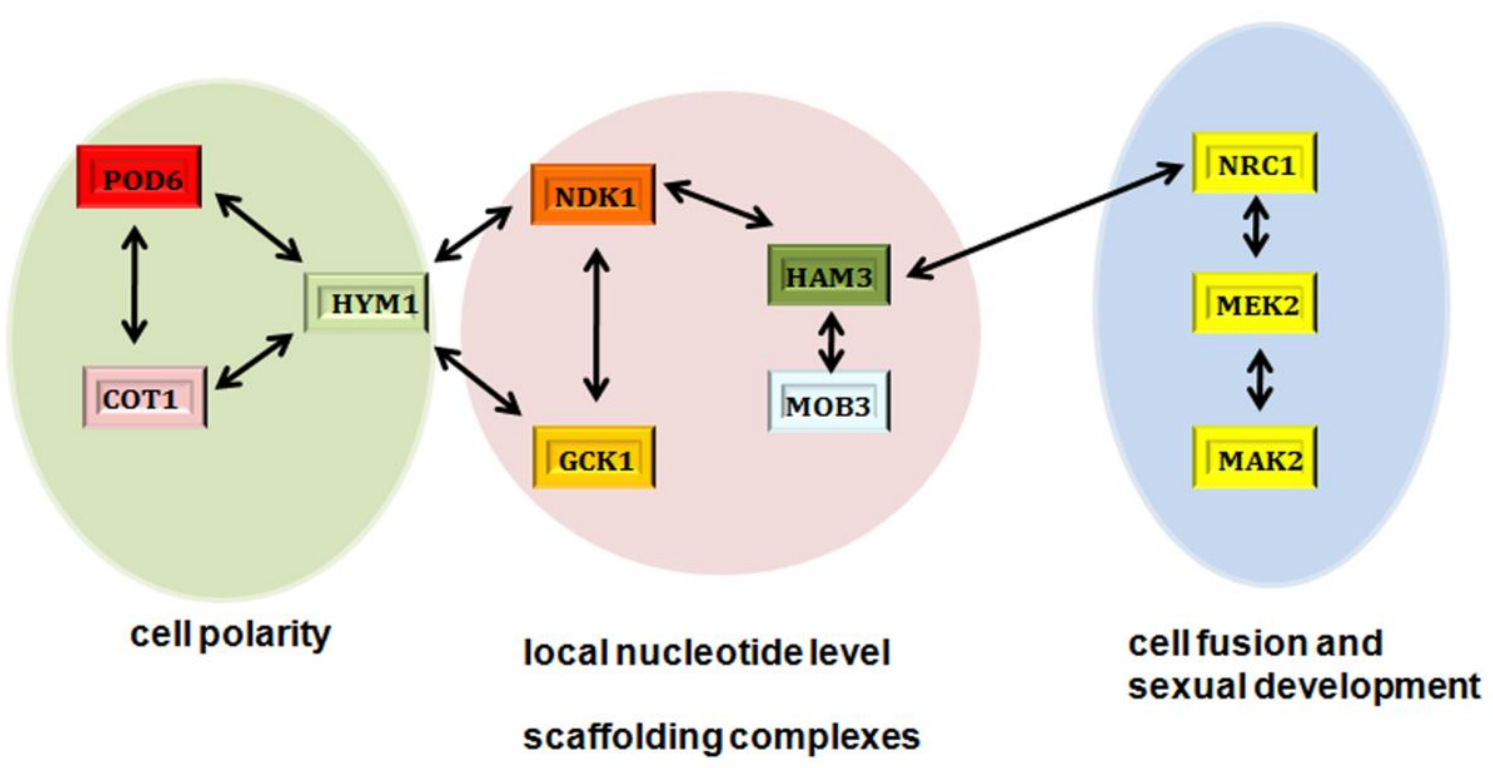

Figure 34: First model of the regulation and communication between the NDR COT1 signaling, the MAK2 MAP kinase pathway and the striatin complex. 


\section{Supplemental material}
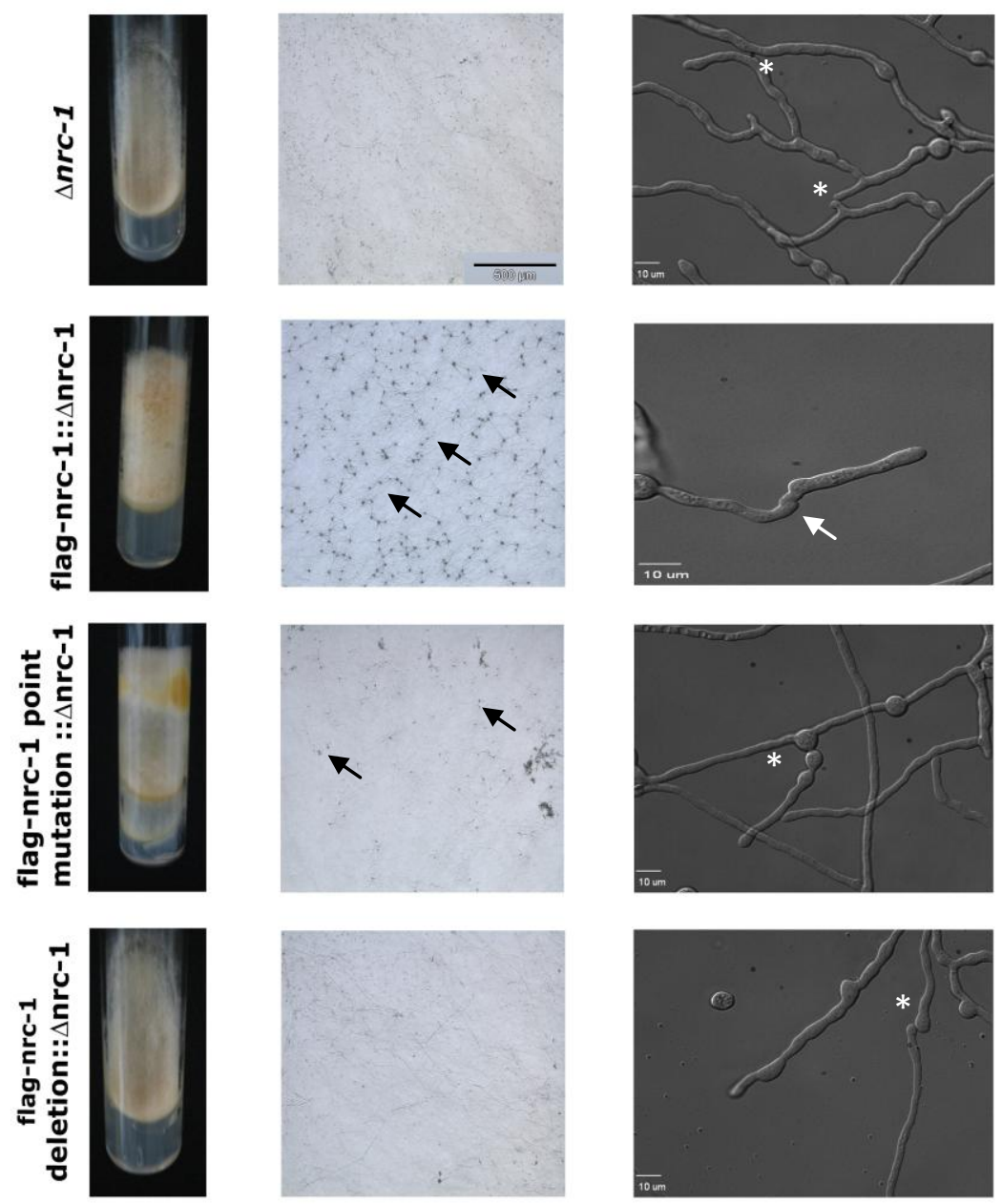

Supplementary Figure 1: Dominant-active NRC1 constructs in $\Delta$ nrc-1 background. Phenotypic characterization with regard to colony morphology (growth for 5 days on minimal medium; right images) and protoperithecia formation (marked by black arrows; growth for 7 days on cornmeal medium; bar $=500 \mu \mathrm{m}$; middle images) and hyphal fusion (fusion marked by white arrows and non-fused cells marked by asterisks; bar $=10 \mu \mathrm{m}$; left images). 

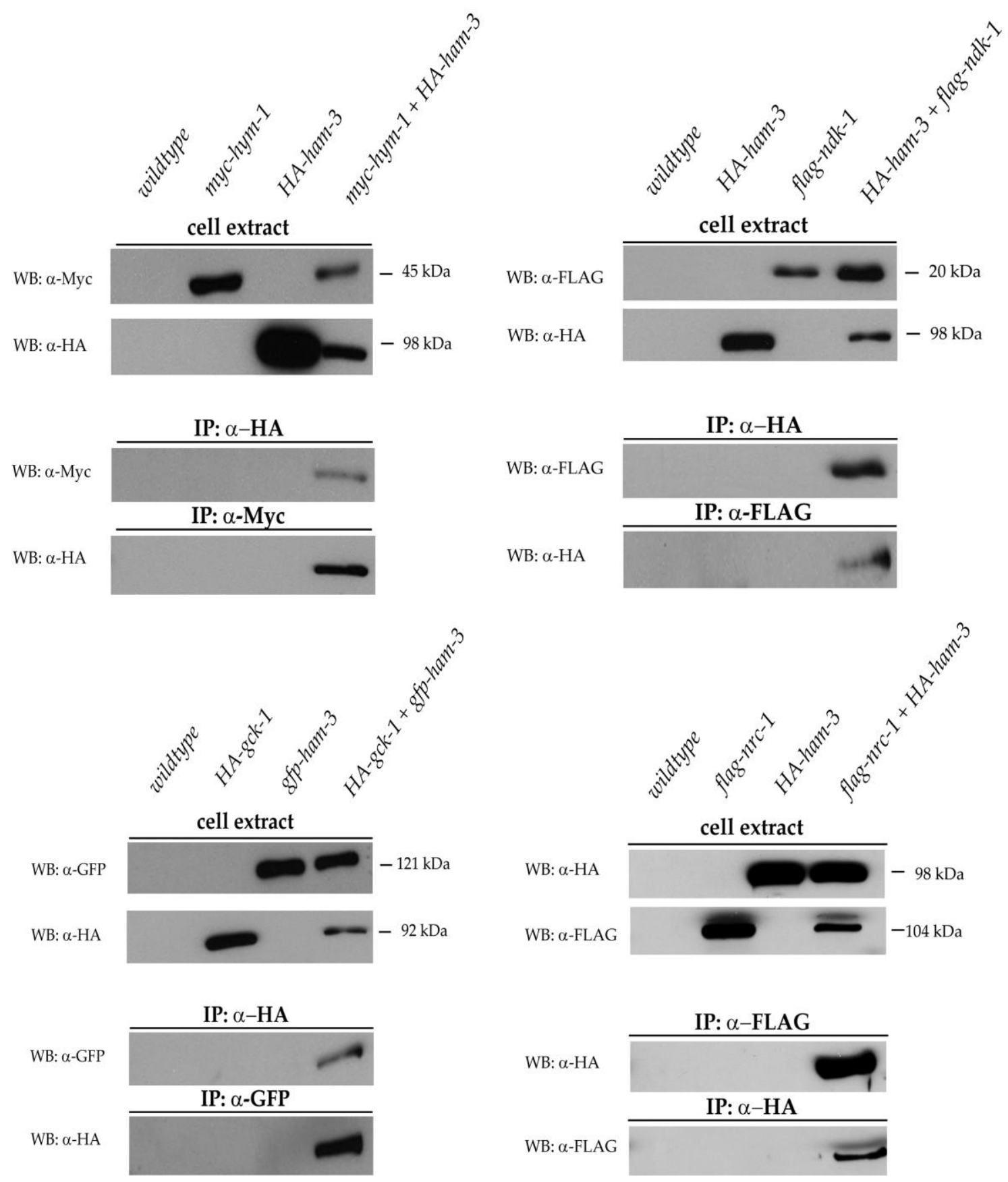

Supplementary Figure 2: Results of co-immunoprecipitation experiments with HAM3 (striatin homologue in N. crassa). Interactions could be identified between HAM3 with GCK1, HYM1, NDK1 and NRC1. 

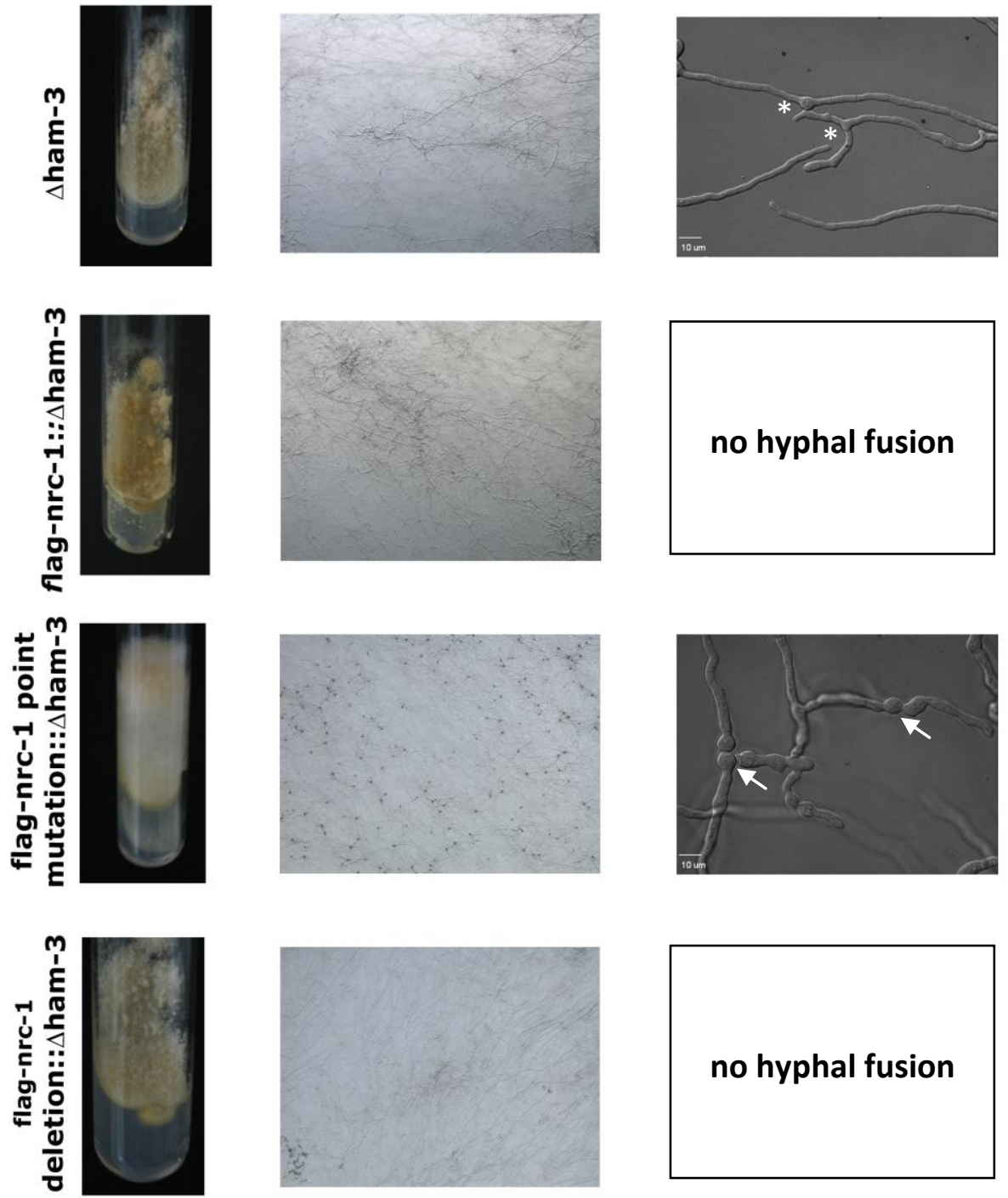

Supplementary Figure 3: Dominant-active NRC1 constructs in $\Delta$ ham-3 background. Phenotypic characterization with regard to colony morphology (growth for 5 days on minimal medium; right images) and protoperithecia formation (marked by black arrows; growth for 7 days on cornmeal medium; bar = 500 $\mu \mathrm{m}$; middle images) and hyphal fusion (marked by white arrows; bar = 10 $\mu \mathrm{m}$; left images).

Supplementary Table 1: Overview of the kinase-substrate pairs tested in several kinase activity assays.

\begin{tabular}{|c|c|c|}
\hline kinase & substrate & phosphorylation \\
\hline \multirow[t]{3}{*}{ NDK1 } & HAM3 & no \\
\hline & МOB3 & no \\
\hline & HYM3 & no \\
\hline \multirow[t]{3}{*}{ GCK1 } & HAM3 & no \\
\hline & МOB3 & no \\
\hline & HYM3 & no \\
\hline \multirow[t]{3}{*}{ NRC1 } & HAM3 & no \\
\hline & МOB3 & no \\
\hline & HYM3 & no \\
\hline \multirow[t]{3}{*}{ COT1 } & HAM3 & yes \\
\hline & MOB3 & no \\
\hline & HYM3 & no \\
\hline
\end{tabular}



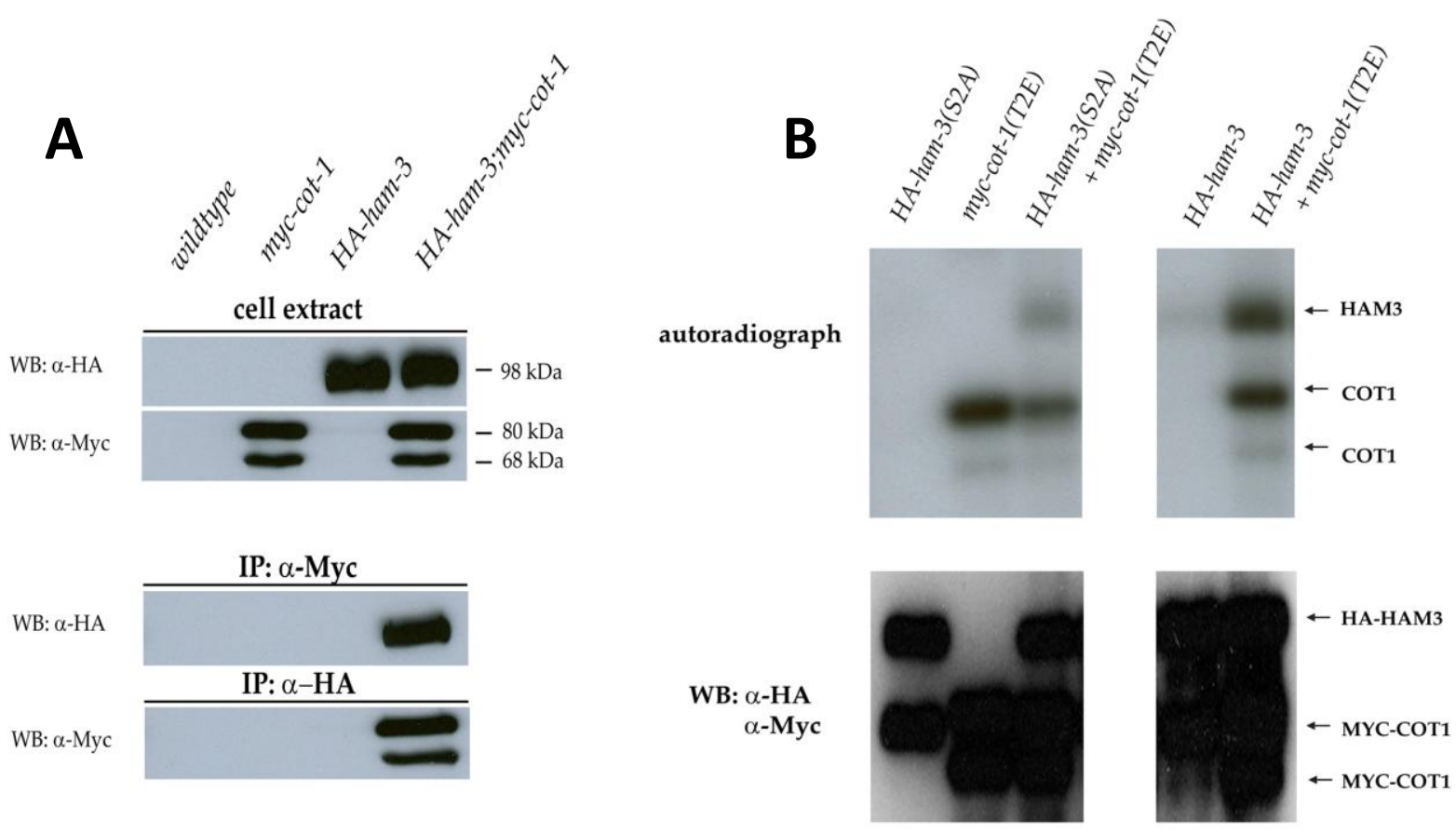

Supplementary Figure 4: Characterization of the COT1-HAM3 interaction.

(A) Co-immunoprecipitation studies in $\mathbf{N}$. crassa exhibit an interaction between myc-tagged COT1 and HAtagged HAM3.

(B) Radioactive in vitro kinase substrate assay showed phosphorylation of HAM3 by the NDR kinase COT1. If the serine residue 409 is mutated to alanine no phosphorylation event could be observed. 


\section{References}

Amendola, R., R. Martinez, A. Negroni, D. Venturelli, B. Tanno, B. Calabretta \& G. Raschella, (2001) DR-nm23 expression affects neuroblastoma cell differentiation, integrin expression, and adhesion characteristics. Med Pediatr Oncol 36: 93-96.

Aramayo, R. \& R. L. Metzenberg, (1996) Gene replacements at the his-3 locus of Neurospora crassa. Fungal Genetics Newsletter 43: 9-13.

Ausubel, F. M., R. Brent, R. E. Kingston, D. D. Moore, J. G. Seidman, J. A. Smith \& K. e. and Struhl, (2002) Short protocols in molecular biology: a compendium of methods from current protocols in molecular biology 5th ed. (New York: Wiley).

Baas, A. F., J. Boudeau, G. P. Sapkota, L. Smit, R. Medema, N. A. Morrice, D. R. Alessi \& H. C. Clevers, (2003) Activation of the tumour suppressor kinase LKB1 by the STE20-like pseudokinase STRAD. EMBO J 22: 3062-3072.

Bahn, Y. S., C. Xue, A. Idnurm, J. C. Rutherford, J. Heitman \& M. E. Cardenas, (2007) Sensing the environment: lessons from fungi. Nat Rev Microbiol 5: 57-69.

Baillat, G., S. Gaillard, F. Castets \& A. Monneron, (2002) Interactions of phocein with nucleoside-diphosphate kinase, Eps15, and Dynamin I. J Biol Chem 277: 18961-18966.

Baillat, G., A. Moqrich, F. Castets, A. Baude, Y. Bailly, A. Benmerah \& A. Monneron, (2001) Molecular cloning and characterization of phocein, a protein found from the Golgi complex to dendritic spines. Mol Biol Cell 12: 663-673.

Bardin, A. J. \& A. Amon, (2001) Men and sin: what's the difference? Nat Rev Mol Cell Biol 2: 815-826.

Bardwell, L., (2005) A walk-through of the yeast mating pheromone response pathway. Peptides 26: 339-350.

Bartoli, M., A. Monneron \& D. Ladant, (1998) Interaction of calmodulin with striatin, a WD-repeat protein present in neuronal dendritic spines. J Biol Chem 273: 22248-22253.

Bartoli, M., J. P. Ternaux, C. Forni, P. Portalier, P. Salin, M. Amalric \& A. Monneron, (1999) Down-regulation of striatin, a neuronal calmodulin-binding protein, impairs rat locomotor activity. J Neurobiol 40: 234243.

Benoist, M., S. Gaillard \& F. Castets, (2006) The striatin family: a new signaling platform in dendritic spines. J Physiol Paris 99: 146-153.

Bernhards, Y. \& S. Poeggeler, (2011) The phocein homologue SmMOB3 is essential for vegetative cell fusion and sexual development in the filamentous ascomycete Sordaria macrospora. Curr Genet 57: 133-149.

Bertani, G., (1951) Studies on Iysogenesis. I. The mode of phage liberation by Iysogenic Escherichia coli. J Bacteriol 62: 293-300.

Bhattacharyya, R. P., A. Remenyi, M. C. Good, C. J. Bashor, A. M. Falick \& W. A. Lim, (2006) The Ste5 scaffold allosterically modulates signaling output of the yeast mating pathway. Science 311: 822-826.

Bichsel, S. J., R. Tamaskovic, M. R. Stegert \& B. A. Hemmings, (2004) Mechanism of activation of NDR (nuclear Dbf2-related) protein kinase by the hMOB1 protein. J Biol Chem 279: 35228-35235.

Bidlingmaier, S., E. L. Weiss, C. Seidel, D. G. Drubin \& M. Snyder, (2001) The Cbk1p pathway is important for polarized cell growth and cell separation in Saccharomyces cerevisiae. Mol Cell Biol 21: 2449-2462.

Biondi, R. M. \& A. R. Nebreda, (2003) Signalling specificity of Ser/Thr protein kinases through docking-sitemediated interactions. Biochem J 372: 1-13.

Blondeau, C., S. Gaillard, J. P. Ternaux, A. Monneron \& A. Baude, (2003) Expression and distribution of phocein and members of the striatin family in neurones of rat peripheral ganglia. Histochem Cell Biol 119: 131138.

Bogomolnaya, L. M., R. Pathak, J. Guo \& M. Polymenis, (2006) Roles of the RAM signaling network in cell cycle progression in Saccharomyces cerevisiae. Curr Genet 49: 384-392.

Borden, K. L., (2000) RING domains: master builders of molecular scaffolds? J Mol Biol 295: 1103-1112.

Borges, M. I., M. O. Azevedo, R. Bonatelli Jr. \& M. S. S. Felipe, and Astolfi-Filho, S., (1990) A practical method for the preparation of total DNA from filamentous fungi. Fungal Genetics Newsletter 37.

Borkovich, K. A., L. A. Alex, O. Yarden, M. Freitag, G. E. Turner, N. D. Read, S. Seiler, D. Bell-Pedersen, J. Paietta, N. Plesofsky, M. Plamann, M. Goodrich-Tanrikulu, U. Schulte, G. Mannhaupt, F. E. Nargang, A. Radford, C. Selitrennikoff, J. E. Galagan, J. C. Dunlap, J. J. Loros, D. Catcheside, H. Inoue, R. Aramayo, M. Polymenis, E. U. Selker, M. S. Sachs, G. A. Marzluf, I. Paulsen, R. Davis, D. J. Ebbole, A. Zelter, E. R. Kalkman, R. O'Rourke, F. Bowring, J. Yeadon, C. Ishii, K. Suzuki, W. Sakai \& R. Pratt, (2004) Lessons from the genome sequence of Neurospora crassa: tracing the path from genomic blueprint to multicellular organism. Microbiol Mol Biol Rev 68: 1-108. 
Boudeau, J., A. F. Baas, M. Deak, N. A. Morrice, A. Kieloch, M. Schutkowski, A. R. Prescott, H. C. Clevers \& D. R. Alessi, (2003a) MO25alpha/beta interact with STRADalpha/beta enhancing their ability to bind, activate and localize LKB1 in the cytoplasm. EMBO J 22: 5102-5114.

Boudeau, J., A. Kieloch, D. R. Alessi, A. Stella, G. Guanti \& N. Resta, (2003b) Functional analysis of LKB1/STK11 mutants and two aberrant isoforms found in Peutz-Jeghers Syndrome patients. Hum Mutat 21: 172.

Boudeau, J., D. Miranda-Saavedra, G. J. Barton \& D. R. Alessi, (2006) Emerging roles of pseudokinases. Trends Cell Biol 16: 443-452.

Boudeau, J., G. Sapkota \& D. R. Alessi, (2003c) LKB1, a protein kinase regulating cell proliferation and polarity. FEBS Lett 546: 159-165.

Boudeau, J., J. W. Scott, N. Resta, M. Deak, A. Kieloch, D. Komander, D. G. Hardie, A. R. Prescott, D. M. van Aalten \& D. R. Alessi, (2004) Analysis of the LKB1-STRAD-MO25 complex. J Cell Sci 117: 6365-6375.

Brewster, J. L., T. de Valoir, N. D. Dwyer, E. Winter \& M. C. Gustin, (1993) An osmosensing signal transduction pathway in yeast. Science 259: 1760-1763.

Brockman, H. E. \& F. J. de Serress, (1963) "Sorbose Toxicity" in Neurospora. American Journal of Botany 50.

Burack, W. R. \& A. S. Shaw, (2000) Signal transduction: hanging on a scaffold. Curr Opin Cell Biol 12: 211-216.

Bussink, H. J. \& S. A. Osmani, (1999) A mitogen-activated protein kinase (MPKA) is involved in polarized growth in the filamentous fungus, Aspergillus nidulans. FEMS Microbiol Lett 173: 117-125.

Castets, F., M. Bartoli, J. V. Barnier, G. Baillat, P. Salin, A. Moqrich, J. P. Bourgeois, F. Denizot, G. Rougon, G. Calothy \& A. Monneron, (1996) A novel calmodulin-binding protein, belonging to the WD-repeat family, is localized in dendrites of a subset of CNS neurons. J Cell Biol 134: 1051-1062.

Castets, F., T. Rakitina, S. Gaillard, A. Moqrich, M. G. Mattei \& A. Monneron, (2000) Zinedin, SG2NA, and striatin are calmodulin-binding, WD repeat proteins principally expressed in the brain. J Biol Chem 275: 1997019977.

Chan, J., R. L. Hui \& E. Levin, (2005) Differential association between statin exposure and elevated levels of creatine kinase. Ann Pharmacother 39: 1611-1616.

Chang, L. \& M. Karin, (2001) Mammalian MAP kinase signalling cascades. Nature 410: 37-40.

Chen, B. C. \& W. W. Lin, (2001) PKC- and ERK-dependent activation of I kappa B kinase by lipopolysaccharide in macrophages: enhancement by P2Y receptor-mediated CaMK activation. Br J Pharmacol 134: 10551065.

Chen, H. W., M. J. Marinissen, S. W. Oh, X. Chen, M. Melnick, N. Perrimon, J. S. Gutkind \& S. X. Hou, (2002) CKA, a novel multidomain protein, regulates the JUN N-terminal kinase signal transduction pathway in Drosophila. Mol Cell Biol 22: 1792-1803.

Chen, W., Q. Tang, M. S. Gonzales \& G. T. Bowden, (2001) Role of p38 MAP kinases and ERK in mediating ultraviolet-B induced cyclooxygenase-2 gene expression in human keratinocytes. Oncogene 20: 39213926.

Chiadmi, M., S. Morera, I. Lascu, C. Dumas, G. Le Bras, M. Veron \& J. Janin, (1993) Crystal structure of the Awd nucleotide diphosphate kinase from Drosophila. Structure 1: 283-293.

Choi, G., H. Yi, J. Lee, Y. K. Kwon, M. S. Soh, B. Shin, Z. Luka, T. R. Hahn \& P. S. Song, (1999) Phytochrome signalling is mediated through nucleoside diphosphate kinase 2. Nature 401: 610-613.

Choi, K. Y., B. Satterberg, D. M. Lyons \& E. A. Elion, (1994) Ste5 tethers multiple protein kinases in the MAP kinase cascade required for mating in S. cerevisiae. Cell 78: 499-512.

Chou, J. J., S. Li, C. B. Klee \& A. Bax, (2001) Solution structure of $\mathrm{Ca}(2+)$-calmodulin reveals flexible hand-like properties of its domains. Nat Struct Biol 8: 990-997.

Cid, V. J., A. Duran, F. del Rey, M. P. Snyder, C. Nombela \& M. Sanchez, (1995) Molecular basis of cell integrity and morphogenesis in Saccharomyces cerevisiae. Microbiol Rev 59: 345-386.

Cobb, M. H., (1999) MAP kinase pathways. Prog Biophys Mol Biol 71: 479-500.

Colman-Lerner, A., T. E. Chin \& R. Brent, (2001) Yeast Cbk1 and Mob2 activate daughter-specific genetic programs to induce asymmetric cell fates. Cell 107: 739-750.

Colomer, J. \& A. R. Means, (2007) Physiological roles of the Ca2+/CaM-dependent protein kinase cascade in health and disease. Subcell Biochem 45: 169-214.

Cote, P., T. Sulea, D. Dignard, C. Wu \& M. Whiteway, (2011) Evolutionary reshaping of fungal mating pathway scaffold proteins. MBio 2.

Couet, J., S. Li, T. Okamoto, T. Ikezu \& M. P. Lisanti, (1997a) Identification of peptide and protein ligands for the caveolin-scaffolding domain. Implications for the interaction of caveolin with caveolae-associated proteins. J Biol Chem 272: 6525-6533.

Couet, J., M. Sargiacomo \& M. P. Lisanti, (1997b) Interaction of a receptor tyrosine kinase, EGF-R, with caveolins. Caveolin binding negatively regulates tyrosine and serine/threonine kinase activities. J Biol Chem 272: 30429-30438. 
Cybulsky, A. V., T. Takano, J. Guillemette, J. Papillon, R. A. Volpini \& J. A. Di Battista, (2009) The Ste20-like kinase SLK promotes p53 transactivation and apoptosis. Am J Physiol Renal Physiol 297: F971-980.

D'Souza, C. A., J. A. Alspaugh, C. Yue, T. Harashima, G. M. Cox, J. R. Perfect \& J. Heitman, (2001) Cyclic AMPdependent protein kinase controls virulence of the fungal pathogen Cryptococcus neoformans. Mol Cell Biol 21: 3179-3191.

Dan, I., N. M. Watanabe \& A. Kusumi, (2001) The Ste20 group kinases as regulators of MAP kinase cascades. Trends Cell Biol 11: 220-230.

Davis, B. J., (1964) Disc Electrophoresis. Ii. Method and Application to Human Serum Proteins. Ann N Y Acad Sci 121: 404-427.

Davis, R. H., (2000) Neurospora: contributions of a model organism. (Oxford University Press).

Davis, R. H., de Serres, F.J., (1970) Genetic and microbiological research techniques for Neurospora crassa. . Methods in Enzymology 17: 79-143.

de Bettignies, G. \& L. H. Johnston, (2003) The mitotic exit network. Curr Biol 13: R301.

Dunlap, J. C., K. A. Borkovich, M. R. Henn, G. E. Turner, M. S. Sachs, N. L. Glass, K. McCluskey, M. Plamann, J. E. Galagan, B. W. Birren, R. L. Weiss, J. P. Townsend, J. J. Loros, M. A. Nelson, R. Lambreghts, H. V. Colot, G. Park, P. Collopy, C. Ringelberg, C. Crew, L. Litvinkova, D. DeCaprio, H. M. Hood, S. Curilla, M. Shi, M. Crawford, M. Koerhsen, P. Montgomery, L. Larson, M. Pearson, T. Kasuga, C. Tian, M. Basturkmen, L. Altamirano \& J. Xu, (2007) Enabling a community to dissect an organism: overview of the Neurospora functional genomics project. Adv Genet 57: 49-96.

Durrenberger, F. \& J. Kronstad, (1999) The ukc1 gene encodes a protein kinase involved in morphogenesis, pathogenicity and pigment formation in Ustilago maydis. Mol Gen Genet 261: 281-289.

Elbing, K., R. R. McCartney \& M. C. Schmidt, (2006a) Purification and characterization of the three Snf1activating kinases of Saccharomyces cerevisiae. Biochem J 393: 797-805.

Elbing, K., E. M. Rubenstein, R. R. McCartney \& M. C. Schmidt, (2006b) Subunits of the Snf1 kinase heterotrimer show interdependence for association and activity. J Biol Chem 281: 26170-26180.

Elion, E. A., (1995) Ste5: a meeting place for MAP kinases and their associates. Trends Cell Biol 5: 322-327.

Elion, E. A., (1998) Routing MAP kinase cascades. Science 281: 1625-1626.

Elion, E. A., M. Qi \& W. Chen, (2005) Signal transduction. Signaling specificity in yeast. Science 307: 687-688.

Emoto, K., J. Z. Parrish, L. Y. Jan \& Y. N. Jan, (2006) The tumour suppressor Hippo acts with the NDR kinases in dendritic tiling and maintenance. Nature 443: 210-213.

English, J. M. \& M. H. Cobb, (2002) Pharmacological inhibitors of MAPK pathways. Trends Pharmacol Sci 23: 4045.

Enomoto, A., N. Kido, M. Ito, A. Morita, Y. Matsumoto, N. Takamatsu, Y. Hosoi \& K. Miyagawa, (2008) Negative regulation of MEKK1/2 signaling by serine-threonine kinase 38 (STK38). Oncogene 27: 1930-1938.

Fields, S. \& O. Song, (1989) A novel genetic system to detect protein-protein interactions. Nature 340: 245-246.

Filippi, B. M., P. de los Heros, Y. Mehellou, I. Navratilova, R. Gourlay, M. Deak, L. Plater, R. Toth, E. Zeqiraj \& D. R. Alessi, $(2011$

) MO25 is a master regulator of SPAK/OSR1 and MST3/MST4/YSK1 protein kinases. EMBO J 30: 1730-1741.

Fischer, R., N. Zekert \& N. Takeshita, (2008) Polarized growth in fungi--interplay between the cytoskeleton, positional markers and membrane domains. Mol Microbiol 68: 813-826.

Fleissner, A., S. Diamond \& N. L. Glass, (2009a) The Saccharomyces cerevisiae PRM1 homolog in Neurospora crassa is involved in vegetative and sexual cell fusion events but also has postfertilization functions. Genetics 181: 497-510.

Fleissner, A., A. C. Leeder, M. G. Roca, N. D. Read \& N. L. Glass, (2009b) Oscillatory recruitment of signaling proteins to cell tips promotes coordinated behavior during cell fusion. Proc Natl Acad Sci U S A 106: 19387-19392.

Freitag, M., P. C. Hickey, N. B. Raju, E. U. Selker \& N. D. Read, (2004) GFP as a tool to analyze the organization, dynamics and function of nuclei and microtubules in Neurospora crassa. Fungal Genet Biol 41: 897910.

Frodin, M., T. L. Antal, B. A. Dummler, C. J. Jensen, M. Deak, S. Gammeltoft \& R. M. Biondi, (2002) A phosphoserine/threonine-binding pocket in AGC kinases and PDK1 mediates activation by hydrophobic motif phosphorylation. EMBO J 21: 5396-5407.

Fujimura, M., N. Ochiai, M. Oshima, T. Motoyama, A. Ichiishi, R. Usami, K. Horikoshi \& I. Yamaguchi, (2003) Putative homologs of SSK22 MAPKK kinase and PBS2 MAPK kinase of Saccharomyces cerevisiae encoded by os-4 and os-5 genes for osmotic sensitivity and fungicide resistance in Neurospora crassa. Biosci Biotechnol Biochem 67: 186-191.

Fukuchi, T., J. Nikawa, N. Kimura \& K. Watanabe, (1993) Isolation, overexpression and disruption of a Saccharomyces cerevisiae YNK gene encoding nucleoside diphosphate kinase. Gene 129: 141-146. 
Furukawa, K., Y. Hoshi, T. Maeda, T. Nakajima \& K. Abe, (2005) Aspergillus nidulans HOG pathway is activated only by two-component signalling pathway in response to osmotic stress. Mol Microbiol 56: 12461261.

Gaillard, S., Y. Bailly, M. Benoist, T. Rakitina, J. P. Kessler, L. Fronzaroli-Molinieres, B. Dargent \& F. Castets, (2006) Targeting of proteins of the striatin family to dendritic spines: role of the coiled-coil domain. Traffic 7: 74-84.

Gaillard, S., M. Bartoli, F. Castets \& A. Monneron, (2001) Striatin, a calmodulin-dependent scaffolding protein, directly binds caveolin-1. FEBS Lett 508: 49-52.

Galagan, J. E., S. E. Calvo, C. Cuomo, L. J. Ma, J. R. Wortman, S. Batzoglou, S. I. Lee, M. Basturkmen, C. C. Spevak, J. Clutterbuck, V. Kapitonov, J. Jurka, C. Scazzocchio, M. Farman, J. Butler, S. Purcell, S. Harris, G. H. Braus, O. Draht, S. Busch, C. D'Enfert, C. Bouchier, G. H. Goldman, D. Bell-Pedersen, S. Griffiths-Jones, J. H. Doonan, J. Yu, K. Vienken, A. Pain, M. Freitag, E. U. Selker, D. B. Archer, M. A. Penalva, B. R. Oakley, M. Momany, T. Tanaka, T. Kumagai, K. Asai, M. Machida, W. C. Nierman, D. W. Denning, M. Caddick, M. Hynes, M. Paoletti, R. Fischer, B. Miller, P. Dyer, M. S. Sachs, S. A. Osmani \& B. W. Birren, (2005a) Sequencing of Aspergillus nidulans and comparative analysis with A. fumigatus and A. oryzae. Nature 438: 1105-1115.

Galagan, J. E., M. R. Henn, L. J. Ma, C. A. Cuomo \& B. Birren, (2005b) Genomics of the fungal kingdom: insights into eukaryotic biology. Genome Res 15: 1620-1631.

Geng, W., B. He, M. Wang \& P. N. Adler, (2000) The tricornered gene, which is required for the integrity of epidermal cell extensions, encodes the Drosophila nuclear DBF2-related kinase. Genetics 156: 18171828.

Good, M., G. Tang, J. Singleton, A. Remenyi \& W. A. Lim, (2009) The Ste5 scaffold directs mating signaling by catalytically unlocking the Fus3 MAP kinase for activation. Cell 136: 1085-1097.

Gorovits, R. \& O. Yarden, (2003) Environmental suppression of Neurospora crassa cot-1 hyperbranching: a link between COT1 kinase and stress sensing. Eukaryot Cell 2: 699-707.

Goshima, T., K. Kume, T. Koyano, Y. Ohya, T. Toda \& D. Hirata, (2010) Fission yeast germinal center (GC) kinase Ppk11 interacts with Pmo25 and plays an auxiliary role in concert with the morphogenesis Orb6 network (MOR) in cell morphogenesis. J Biol Chem 285: 35196-35205.

Goudreault, M., L. M. D'Ambrosio, M. J. Kean, M. J. Mullin, B. G. Larsen, A. Sanchez, S. Chaudhry, G. I. Chen, F. Sicheri, A. I. Nesvizhskii, R. Aebersold, B. Raught \& A. C. Gingras, (2009) A PP2A phosphatase high density interaction network identifies a novel striatin-interacting phosphatase and kinase complex linked to the cerebral cavernous malformation 3 (CCM3) protein. Mol Cell Proteomics 8: 157-171.

Gustin, M. C., J. Albertyn, M. Alexander \& K. Davenport, (1998) MAP kinase pathways in the yeast Saccharomyces cerevisiae. Microbiol Mol Biol Rev 62: 1264-1300.

Hanks, S. K. \& T. Hunter, (1995) Protein kinases 6. The eukaryotic protein kinase superfamily: kinase (catalytic) domain structure and classification. FASEB J 9: 576-596.

Harris, S. D., (2006) Cell polarity in filamentous fungi: shaping the mold. Int Rev Cytol 251: 41-77.

Harris, S. D. \& M. Momany, (2004) Polarity in filamentous fungi: moving beyond the yeast paradigm. Fungal Genet Biol 41: 391-400.

Hasunuma, K. \& N. Yabe, (1998) [Early events occurring during light signal transduction in plants]. Tanpakushitsu Kakusan Koso 43: 1443-1452.

He, Y., K. Emoto, X. Fang, N. Ren, X. Tian, Y. N. Jan \& P. N. Adler, (2005) Drosophila Mob family proteins interact with the related tricornered $(\mathrm{TrC})$ and warts (Wts) kinases. Mol Biol Cell 16: 4139-4152.

Hedbacker, K. \& M. Carlson, (2008) SNF1/AMPK pathways in yeast. Front Biosci 13: 2408-2420.

Hergovich, A., S. J. Bichsel \& B. A. Hemmings, (2005) Human NDR kinases are rapidly activated by MOB proteins through recruitment to the plasma membrane and phosphorylation. Mol Cell Biol 25: 8259-8272.

Hergovich, A., D. Schmitz \& B. A. Hemmings, (2006a) The human tumour suppressor LATS1 is activated by human MOB1 at the membrane. Biochem Biophys Res Commun 345: 50-58.

Hergovich, A., M. R. Stegert, D. Schmitz \& B. A. Hemmings, (2006b) NDR kinases regulate essential cell processes from yeast to humans. Nat Rev Mol Cell Biol 7: 253-264.

Hickey, P. C., D. Jacobson, N. D. Read \& N. L. Louise Glass, (2002) Live-cell imaging of vegetative hyphal fusion in Neurospora crassa. Fungal Genet Biol 37: 109-119.

Hirata, D., N. Kishimoto, M. Suda, Y. Sogabe, S. Nakagawa, Y. Yoshida, K. Sakai, M. Mizunuma, T. Miyakawa, J. Ishiguro \& T. Toda, (2002) Fission yeast Mor2/Cps12, a protein similar to Drosophila Furry, is essential for cell morphogenesis and its mutation induces Wee1-dependent G(2) delay. EMBO J 21: 4863-4874.

Ho, L. L., X. Wei, T. Shimizu \& Z. C. Lai, (2010) Mob as tumor suppressor is activated at the cell membrane to control tissue growth and organ size in Drosophila. Dev Biol 337: 274-283. 
Ho, Y., A. Gruhler, A. Heilbut, G. D. Bader, L. Moore, S. L. Adams, A. Millar, P. Taylor, K. Bennett, K. Boutilier, L. Yang, C. Wolting, I. Donaldson, S. Schandorff, J. Shewnarane, M. Vo, J. Taggart, M. Goudreault, B. Muskat, C. Alfarano, D. Dewar, Z. Lin, K. Michalickova, A. R. Willems, H. Sassi, P. A. Nielsen, K. J. Rasmussen, J. R. Andersen, L. E. Johansen, L. H. Hansen, H. Jespersen, A. Podtelejnikov, E. Nielsen, J. Crawford, V. Poulsen, B. D. Sorensen, J. Matthiesen, R. C. Hendrickson, F. Gleeson, T. Pawson, M. F. Moran, D. Durocher, M. Mann, C. W. Hogue, D. Figeys \& M. Tyers, (2002) Systematic identification of protein complexes in Saccharomyces cerevisiae by mass spectrometry. Nature 415: 180-183.

Honda, S. \& E. U. Selker, (2009) Tools for fungal proteomics: multifunctional neurospora vectors for gene replacement, protein expression and protein purification. Genetics 182: 11-23.

Hou, M. C., D. A. Guertin \& D. McCollum, (2004) Initiation of cytokinesis is controlled through multiple modes of regulation of the Sid2p-Mob1p kinase complex. Mol Cell Biol 24: 3262-3276.

Hou, M. C., D. J. Wiley, F. Verde \& D. McCollum, (2003) Mob2p interacts with the protein kinase Orb6p to promote coordination of cell polarity with cell cycle progression. J Cell Sci 116: 125-135.

Hou, Z., C. Xue, Y. Peng, T. Katan, H. C. Kistler \& J. R. Xu, (2002) A mitogen-activated protein kinase gene (MGV1) in Fusarium graminearum is required for female fertility, heterokaryon formation, and plant infection. Mol Plant Microbe Interact 15: 1119-1127.

Huang, L. S., H. K. Doherty \& I. Herskowitz, (2005) The Smk1p MAP kinase negatively regulates Gsc2p, a 1,3beta-glucan synthase, during spore wall morphogenesis in Saccharomyces cerevisiae. Proc Natl Acad Sci U S A 102: 12431-12436.

Ito, T., T. Chiba, R. Ozawa, M. Yoshida, M. Hattori \& Y. Sakaki, (2001) A comprehensive two-hybrid analysis to explore the yeast protein interactome. Proc Natl Acad Sci U S A 98: 4569-4574.

Izumiya, H. \& M. Yamamoto, (1995) Cloning and functional analysis of the ndk1 gene encoding nucleosidediphosphate kinase in Schizosaccharomyces pombe. J Biol Chem 270: 27859-27864.

James, P., J. Halladay \& E. A. Craig, (1996) Genomic libraries and a host strain designed for highly efficient twohybrid selection in yeast. Genetics 144: 1425-1436.

Jansen, J. M., M. F. Barry, C. K. Yoo \& E. L. Weiss, (2006) Phosphoregulation of Cbk1 is critical for RAM network control of transcription and morphogenesis. J Cell Biol 175: 755-766.

Johns, S. A., A. C. Leeder, M. Safaie \& G. Turner, (2006) Depletion of Aspergillus nidulans cotA causes a severe polarity defect which is not suppressed by the nuclear migration mutation nudA2. Mol Genet Genomics 275: 593-604.

Johnson, G. L. \& R. Lapadat, (2002) Mitogen-activated protein kinase pathways mediated by ERK, JNK, and p38 protein kinases. Science 298: 1911-1912.

Jones, C. A., S. E. Greer-Phillips \& K. A. Borkovich, (2007) The response regulator RRG-1 functions upstream of a mitogen-activated protein kinase pathway impacting asexual development, female fertility, osmotic stress, and fungicide resistance in Neurospora crassa. Mol Biol Cell 18: 2123-2136.

Jourdain, P., K. Fukunaga \& D. Muller, (2003) Calcium/calmodulin-dependent protein kinase II contributes to activity-dependent filopodia growth and spine formation. J Neurosci 23: 10645-10649.

Kanai, M., K. Kume, K. Miyahara, K. Sakai, K. Nakamura, K. Leonhard, D. J. Wiley, F. Verde, T. Toda \& D. Hirata, (2005) Fission yeast MO25 protein is localized at SPB and septum and is essential for cell morphogenesis. EMBO J 24: 3012-3025.

Kannan, N., N. Haste, S. S. Taylor \& A. F. Neuwald, (2007) The hallmark of AGC kinase functional divergence is its C-terminal tail, a cis-acting regulatory module. Proc Natl Acad Sci U S A 104: 1272-1277.

Kawabata, T. \& H. and Inoue, (2007) Detection of physical interactions by immunoprecipitation of FLAG- and HA-tagged proteins expressed at the his-3 locus in Neurospora crassa. Fungal Genetics Newsletter 54: 5-8.

Kawasaki, L., O. Sanchez, K. Shiozaki \& J. Aguirre, (2002) SakA MAP kinase is involved in stress signal transduction, sexual development and spore viability in Aspergillus nidulans. Mol Microbiol 45: 11531163.

Kean, M. J., D. Ceccarelli, M. Goudreault, M. Sanches, S. Tate, B. Larsen, L. C. Gibson, W. B. Derry, I. C. Scott, L. Pelletier, G. S. Baillie, F. Sicheri \& A. C. Gingras, (2011) Structure-function analysis of core STRIPAK: a signalling complex implicated in golgi polarization. J Biol Chem.

Keranen, L. M., E. M. Dutil \& A. C. Newton, (1995) Protein kinase C is regulated in vivo by three functionally distinct phosphorylations. Curr Biol 5: 1394-1403.

Kim, K. Y., A. W. Truman, S. Caesar, G. Schlenstedt \& D. E. Levin, (2010) Yeast Mpk1 cell wall integrity mitogenactivated protein kinase regulates nucleocytoplasmic shuttling of the Swi6 transcriptional regulator. Mol Biol Cell 21: 1609-1619. 
Kojima, K., T. Kikuchi, Y. Takano, E. Oshiro \& T. Okuno, (2002) The mitogen-activated protein kinase gene MAF1 is essential for the early differentiation phase of appressorium formation in Colletotrichum lagenarium. Mol Plant Microbe Interact 15: 1268-1276.

Kothe, G. O. \& S. J. Free, (1998) The isolation and characterization of nrc-1 and nrc-2, two genes encoding protein kinases that control growth and development in Neurospora crassa. Genetics 149: 117-130.

Kranz, J. E., B. Satterberg \& E. A. Elion, (1994) The MAP kinase Fus3 associates with and phosphorylates the upstream signaling component Ste5. Genes Dev 8: 313-327.

Krapp, A., M. P. Gulli \& V. Simanis, (2004) SIN and the art of splitting the fission yeast cell. Curr Biol 14: R722730.

Krapp, A. \& V. Simanis, (2005) Cell division: SIN, cytokinesis and ethanol dependency. Curr Biol 15: R605-607.

Krapp, A. \& V. Simanis, (2008) An overview of the fission yeast septation initiation network (SIN). Biochem Soc Trans 36: 411-415.

Krisak, L., R. Strich, R. S. Winters, J. P. Hall, M. J. Mallory, D. Kreitzer, R. S. Tuan \& E. Winter, (1994) SMK1, a developmentally regulated MAP kinase, is required for spore wall assembly in Saccharomyces cerevisiae. Genes Dev 8: 2151-2161.

Kume, K., T. Goshima, K. Miyahara, T. Toda \& D. Hirata, (2007) A method for Pmo25-associated kinase assay in fission yeast: the activity is dependent on two gC kinases Nak1 and Sid1. Biosci Biotechnol Biochem 71: 615-617.

Kurischko, C., G. Weiss, M. Ottey \& F. C. Luca, (2005) A role for the Saccharomyces cerevisiae regulation of Ace2 and polarized morphogenesis signaling network in cell integrity. Genetics 171: 443-455.

Laemmli, U. K., (1970) Cleavage of structural proteins during the assembly of the head of bacteriophage T4. Nature 227: 680-685.

Lambright, D. G., J. Sondek, A. Bohm, N. P. Skiba, H. E. Hamm \& P. B. Sigler, (1996) The 2.0 A crystal structure of a heterotrimeric G protein. Nature 379: 311-319.

Lee, B., Y. Yoshida \& K. Hasunuma, (2006) Photomorphogenetic characteristics are severely affected in nucleoside diphosphate kinase-1 (ndk-1)-disrupted mutants in Neurospora crassa. Mol Genet Genomics 275: 9-17.

Lee, H. Y. \& H. Lee, (1999) Inhibitory activity of nm23-H1 on invasion and colonization of human prostate carcinoma cells is not mediated by its NDP kinase activity. Cancer Lett 145: 93-99.

Lee, I. H., S. I. Chang, K. Okada, H. Baba \& H. Shiku, (1997) Transcription effect of nm23-M2/NDP kinase on cmyc oncogene. Mol Cells 7: 589-593.

Lengeler, K. B., R. C. Davidson, C. D'Souza, T. Harashima, W. C. Shen, P. Wang, X. Pan, M. Waugh \& J. Heitman, (2000) Signal transduction cascades regulating fungal development and virulence. Microbiol Mol Biol Rev 64: 746-785.

Lewis, T. S., P. S. Shapiro \& N. G. Ahn, (1998) Signal transduction through MAP kinase cascades. Adv Cancer Res 74: 49-139.

Li, D., P. Bobrowicz, H. H. Wilkinson \& D. J. Ebbole, (2005) A mitogen-activated protein kinase pathway essential for mating and contributing to vegetative growth in Neurospora crassa. Genetics 170: 1091-1104.

Lin, L. F. \& R. E. Levin, (1990) Relative effectiveness of yeast cell wall digesting enzymes on Yarrowia lipolytica. Microbios 63: 109-115.

Lu, Q., D. C. Pallas, H. K. Surks, W. E. Baur, M. E. Mendelsohn \& R. H. Karas, (2004) Striatin assembles a membrane signaling complex necessary for rapid, nongenomic activation of endothelial NO synthase by estrogen receptor alpha. Proc Natl Acad Sci U S A 101: 17126-17131.

Luca, F. C. \& M. Winey, (1998) MOB1, an essential yeast gene required for completion of mitosis and maintenance of ploidy. Mol Biol Cell 9: 29-46.

Ma, H. L., Y. L. Peng, L. Gong, W. B. Liu, S. Sun, J. Liu, C. B. Zheng, H. Fu, D. Yuan, J. Zhao, P. C. Chen, S. S. Xie, X. M. Zeng, Y. M. Xiao, Y. Liu \& D. W. Li, (2009) The goldfish SG2NA gene encodes two alpha-type regulatory subunits for PP-2A and displays distinct developmental expression pattern. Gene Regul Syst Bio 3: $115-129$.

Madhani, H. D., (2000) Interplay of intrinsic and extrinsic signals in yeast differentiation. Proc Natl Acad Sci U S A 97: 13461-13463.

Maeda, I., Y. Kohara, M. Yamamoto \& A. Sugimoto, (2001) Large-scale analysis of gene function in Caenorhabditis elegans by high-throughput RNAi. Curr Biol 11: 171-176.

Maeda, T., M. Takekawa \& H. Saito, (1995) Activation of yeast PBS2 MAPKK by MAPKKKs or by binding of an SH3-containing osmosensor. Science 269: 554-558.

Maeda, T., S. M. Wurgler-Murphy \& H. Saito, (1994) A two-component system that regulates an osmosensing MAP kinase cascade in yeast. Nature 369: 242-245. 
Maerz, S., A. Dettmann, C. Ziv, Y. Liu, O. Valerius, O. Yarden \& S. Seiler, (2009) Two NDR kinase-MOB complexes function as distinct modules during septum formation and tip extension in Neurospora crassa. Mol Microbiol 74: 707-723.

Maerz, S. \& S. Seiler, (2010) Tales of RAM and MOR: NDR kinase signaling in fungal morphogenesis. Curr Opin Microbiol 13: 663-671.

Maerz, S., C. Ziv, N. Vogt, K. Helmstaedt, N. Cohen, R. Gorovits, O. Yarden \& S. Seiler, (2008) The nuclear Dbf2related kinase COT1 and the mitogen-activated protein kinases MAK1 and MAK2 genetically interact to regulate filamentous growth, hyphal fusion and sexual development in Neurospora crassa. Genetics 179: 1313-1325.

Maleri, S., Q. Ge, E. A. Hackett, Y. Wang, H. G. Dohlman \& B. Errede, (2004) Persistent activation by constitutive Ste7 promotes Kss1-mediated invasive growth but fails to support Fus3-dependent mating in yeast. Mol Cell Biol 24: 9221-9238.

Malleshaiah, M. K., V. Shahrezaei, P. S. Swain \& S. W. Michnick, (2010) The scaffold protein Ste5 directly controls a switch-like mating decision in yeast. Nature 465: 101-105.

Manning, A. M. \& R. J. Davis, (2003) Targeting JNK for therapeutic benefit: from junk to gold? Nat Rev Drug Discov 2: 554-565.

Manning, G., G. D. Plowman, T. Hunter \& S. Sudarsanam, (2002) Evolution of protein kinase signaling from yeast to man. Trends Biochem Sci 27: 514-520.

Marcus, S., A. Polverino, M. Barr \& M. Wigler, (1994) Complexes between STE5 and components of the pheromone-responsive mitogen-activated protein kinase module. Proc Natl Acad Sci U S A 91: 77627766.

Margolin, B. S., M. Freitag \& E. U. and Selker, (1997) Improved plasmids for gene targeting at the his-3 locus of Neurospora crassa by electroporation. Fungal Genetics Newsletter 44.

Martin, P. \& W. Wood, (2002) Epithelial fusions in the embryo. Curr Opin Cell Biol 14: 569-574.

McNemar, M. D. \& W. A. Fonzi, (2002) Conserved serine/threonine kinase encoded by CBK1 regulates expression of several hypha-associated transcripts and genes encoding cell wall proteins in Candida albicans. J Bacteriol 184: 2058-2061.

Mendoza, M., S. Redemann \& D. Brunner, (2005) The fission yeast MO25 protein functions in polar growth and cell separation. Eur J Cell Biol 84: 915-926.

Merril, C. R., (1990) Gel-staining techniques. Methods Enzymol 182: 477-488.

Mey, G., K. Held, J. Scheffer, K. B. Tenberge \& P. Tudzynski, (2002) CPMK2, an SLT2-homologous mitogenactivated protein (MAP) kinase, is essential for pathogenesis of Claviceps purpurea on rye: evidence for a second conserved pathogenesis-related MAP kinase cascade in phytopathogenic fungi. Mol Microbiol 46: 305-318.

Milburn, C. C., J. Boudeau, M. Deak, D. R. Alessi \& D. M. van Aalten, (2004) Crystal structure of MO25 alpha in complex with the $C$ terminus of the pseudo kinase STE20-related adaptor. Nat Struct Mol Biol 11: 193200.

Millward, T., P. Cron \& B. A. Hemmings, (1995) Molecular cloning and characterization of a conserved nuclear serine(threonine) protein kinase. Proc Natl Acad Sci U S A 92: 5022-5026.

Millward, T. A., C. W. Heizmann, B. W. Schafer \& B. A. Hemmings, (1998) Calcium regulation of Ndr protein kinase mediated by S100 calcium-binding proteins. EMBO J 17: 5913-5922.

Mishra, N. C. \& E. L. Tatum, (1972) Effect of L-sorbose on polysaccharide synthetases of Neurospora crassa (glycogen- -1,3-glucan-morphology-cell wall-digitonin-particulate enzymes). Proc Natl Acad Sci U S A 69: 313-317.

Miyamoto, H., A. Matsushiro \& M. Nozaki, (1993) Molecular cloning of a novel mRNA sequence expressed in cleavage stage mouse embryos. Mol Reprod Dev 34: 1-7.

Momany, M., (2002) Polarity in filamentous fungi: establishment, maintenance and new axes. Curr Opin Microbiol 5: 580-585.

Monge, R. A., E. Roman, C. Nombela \& J. Pla, (2006) The MAP kinase signal transduction network in Candida albicans. Microbiology 152: 905-912.

Moreno, C. S., W. S. Lane \& D. C. Pallas, (2001) A mammalian homolog of yeast MOB1 is both a member and a putative substrate of striatin family-protein phosphatase 2 A complexes. J Biol Chem 276: 2425324260.

Moreno, C. S., S. Park, K. Nelson, D. Ashby, F. Hubalek, W. S. Lane \& D. C. Pallas, (2000) WD40 repeat proteins striatin and $S / G(2)$ nuclear autoantigen are members of a novel family of calmodulin-binding proteins that associate with protein phosphatase 2A. J Biol Chem 275: 5257-5263.

Morrison, D. K. \& R. J. Davis, (2003) Regulation of MAP kinase signaling modules by scaffold proteins in mammals. Annu Rev Cell Dev Biol 19: 91-118. 
Mrkobrada, S., L. Boucher, D. F. Ceccarelli, M. Tyers \& F. Sicheri, (2006) Structural and functional analysis of Saccharomyces cerevisiae Mob1. J Mol Biol 362: 430-440.

Mueller, P., G. Weinzierl, A. Brachmann, M. Feldbrugge \& R. Kahmann, (2003) Mating and pathogenic development of the Smut fungus Ustilago maydis are regulated by one mitogen-activated protein kinase cascade. Eukaryot Cell 2: 1187-1199.

Nelson, B., C. Kurischko, J. Horecka, M. Mody, P. Nair, L. Pratt, A. Zougman, L. D. McBroom, T. R. Hughes, C. Boone \& F. C. Luca, (2003) RAM: a conserved signaling network that regulates Ace $2 p$ transcriptional activity and polarized morphogenesis. Mol Biol Cell 14: 3782-3803.

Noguchi, R., S. Banno, R. Ichikawa, F. Fukumori, A. Ichiishi, M. Kimura, I. Yamaguchi \& M. Fujimura, (2007) Identification of OS-2 MAP kinase-dependent genes induced in response to osmotic stress, antifungal agent fludioxonil, and heat shock in Neurospora crassa. Fungal Genet Biol 44: 208-218.

Nozaki, M., Y. Onishi, S. Togashi \& H. Miyamoto, (1996) Molecular characterization of the Drosophila Mo25 gene, which is conserved among Drosophila, mouse, and yeast. DNA Cell Biol 15: 505-509.

O'Rourke, S. M. \& I. Herskowitz, (1998) The Hog1 MAPK prevents cross talk between the HOG and pheromone response MAPK pathways in Saccharomyces cerevisiae. Genes Dev 12: 2874-2886.

Ornstein, L., (1964) Disc Electrophoresis. I. Background and Theory. Ann N Y Acad Sci 121: 321-349.

Ouatas, T., B. Abdallah, L. Gasmi, J. Bourdais, E. Postel \& A. Mazabraud, (1997) Three different genes encode NM23/nucleoside diphosphate kinases in Xenopus laevis. Gene 194: 215-225.

Ouatas, T., M. Selo, Z. Sadji, J. Hourdry, H. Denis \& A. Mazabraud, (1998) Differential expression of nucleoside diphosphate kinases (NDPK/NM23) during Xenopus early development. Int J Dev Biol 42: 43-52.

Palanivelu, R. \& D. Preuss, (2000) Pollen tube targeting and axon guidance: parallels in tip growth mechanisms. Trends Cell Biol 10: 517-524.

Pan, X., T. Harashima \& J. Heitman, (2000) Signal transduction cascades regulating pseudohyphal differentiation of Saccharomyces cerevisiae. Curr Opin Microbiol 3: 567-572.

Pandey, A., M. G. Roca, N. D. Read \& N. L. Glass, (2004) Role of a mitogen-activated protein kinase pathway during conidial germination and hyphal fusion in Neurospora crassa. Eukaryot Cell 3: 348-358.

Petralia, R. S., Y. X. Wang \& R. J. Wenthold, (2003) Internalization at glutamatergic synapses during development. Eur J Neurosci 18: 3207-3217.

Poeggeler, S. \& U. Kueck, (2004) A WD40 repeat protein regulates fungal cell differentiation and can be replaced functionally by the mammalian homologue striatin. Eukaryot Cell 3: 232-240.

Ponchon, L., C. Dumas, A. V. Kajava, D. Fesquet \& A. Padilla, (2004) NMR solution structure of Mob1, a mitotic exit network protein and its interaction with an NDR kinase peptide. J Mol Biol 337: 167-182.

Posas, F., M. Takekawa \& H. Saito, (1998a) Signal transduction by MAP kinase cascades in budding yeast. Curr Opin Microbiol 1: 175-182.

Posas, F., E. A. Witten \& H. Saito, (1998b) Requirement of STE50 for osmostress-induced activation of the STE11 mitogen-activated protein kinase kinase kinase in the high-osmolarity glycerol response pathway. Mol Cell Biol 18: 5788-5796.

Postel, E. H., B. M. Abramczyk, M. N. Levit \& S. Kyin, (2000a) Catalysis of DNA cleavage and nucleoside triphosphate synthesis by NM23-H2/NDP kinase share an active site that implies a DNA repair function. Proc Natl Acad Sci U S A 97: 14194-14199.

Postel, E. H., S. J. Berberich, J. W. Rooney \& D. M. Kaetzel, (2000b) Human NM23/nucleoside diphosphate kinase regulates gene expression through DNA binding to nuclease-hypersensitive transcriptional elements. J Bioenerg Biomembr 32: 277-284.

Printen, J. A. \& G. F. Sprague, Jr., (1994) Protein-protein interactions in the yeast pheromone response pathway: Ste5p interacts with all members of the MAP kinase cascade. Genetics 138: 609-619.

Qi, M. \& E. A. Elion, (2005) MAP kinase pathways. J Cell Sci 118: 3569-3572.

Racki, W. J., A. M. Becam, F. Nasr \& C. J. Herbert, (2000) Cbk1p, a protein similar to the human myotonic dystrophy kinase, is essential for normal morphogenesis in Saccharomyces cerevisiae. EMBO J 19: 4524-4532.

Ribeiro, P. S., F. Josue, A. Wepf, M. C. Wehr, O. Rinner, G. Kelly, N. Tapon \& M. Gstaiger, (2010) Combined functional genomic and proteomic approaches identify a PP2A complex as a negative regulator of Hippo signaling. Mol Cell 39: 521-534.

Riquelme, M., O. Yarden, S. Bartnicki-Garcia, B. Bowman, E. Castro-Longoria, S. J. Free, A. Fleissner, M. Freitag, R. R. Lew, R. Mourino-Perez, M. Plamann, C. Rasmussen, C. Richthammer, R. W. Roberson, E. SanchezLeon, S. Seiler \& M. K. Watters, (2011) Architecture and development of the Neurospora crassa hypha - a model cell for polarized growth. Fungal Biol 115: 446-474.

Roberts-Galbraith, R. H. \& K. L. Gould, (2008) Stepping into the ring: the SIN takes on contractile ring assembly. Genes Dev 22: 3082-3088. 
Roux, P. P. \& J. Blenis, (2004) ERK and p38 MAPK-activated protein kinases: a family of protein kinases with diverse biological functions. Microbiol Mol Biol Rev 68: 320-344.

Roymans, D., K. Vissenberg, C. De Jonghe, R. Willems, G. Engler, N. Kimura, B. Grobben, P. Claes, J. P. Verbelen, C. Van Broeckhoven \& H. Slegers, (2001) Identification of the tumor metastasis suppressor Nm23$\mathrm{H} 1 / \mathrm{Nm} 23-\mathrm{R} 1$ as a constituent of the centrosome. Exp Cell Res 262: 145-153.

Roymans, D., R. Willems, K. Vissenberg, C. De Jonghe, B. Grobben, P. Claes, I. Lascu, D. Van Bockstaele, J. P. Verbelen, C. Van Broeckhoven \& H. Slegers, (2000) Nucleoside diphosphate kinase beta (Nm23R1/NDPKbeta) is associated with intermediate filaments and becomes upregulated upon cAMPinduced differentiation of rat C6 glioma. Exp Cell Res 261: 127-138.

Saito, H., (2010) Regulation of cross-talk in yeast MAPK signaling pathways. Curr Opin Microbiol 13: 677-683.

Salinovich, O. \& R. C. Montelaro, (1986) Reversible staining and peptide mapping of proteins transferred to nitrocellulose after separation by sodium dodecylsulfate-polyacrylamide gel electrophoresis. Anal Biochem 156: 341-347.

Sambrook, J. \& D. and Russell, (2001) Molecular Cloning: A Laboratory Manual 3rd ed. (New York: Cold Spring Harbor Laboratory Press).

Sanghamitra, M., I. Talukder, N. Singarapu, K. V. Sindhu, S. Kateriya \& S. K. Goswami, (2008) WD-40 repeat protein SG2NA has multiple splice variants with tissue restricted and growth responsive properties. Gene 420: 48-56.

Schaeffer, H. J. \& M. J. Weber, (1999) Mitogen-activated protein kinases: specific messages from ubiquitous messengers. Mol Cell Biol 19: 2435-2444.

Scheffer, J., C. Ziv, O. Yarden \& P. Tudzynski, (2005) The COT1 homologue CPCOT1 regulates polar growth and branching and is essential for pathogenicity in Claviceps purpurea. Fungal Genet Biol 42: 107-118.

Schiestl, R. H. \& R. D. Gietz, (1989) High efficiency transformation of intact yeast cells using single stranded nucleic acids as a carrier. Curr Genet 16: 339-346.

Seeliger, M. A. \& J. Kuriyan, (2009) A MAPK scaffold lends a helping hand. Cell 136: 994-996.

Seiler, S. \& M. Plamann, (2003) The genetic basis of cellular morphogenesis in the filamentous fungus Neurospora crassa. Mol Biol Cell 14: 4352-4364.

Seiler, S., N. Vogt, C. Ziv, R. Gorovits \& O. Yarden, (2006) The STE20/germinal center kinase POD6 interacts with the NDR kinase COT1 and is involved in polar tip extension in Neurospora crassa. Mol Biol Cell 17: 4080-4092.

Shi, J., W. Chen, Q. Liu, S. Chen, H. Hu, G. Turner \& L. Lu, (2008) Depletion of the MobB and CotA complex in Aspergillus nidulans causes defects in polarity maintenance that can be suppressed by the environment stress. Fungal Genet Biol 45: 1570-1581.

Shim, W. B., U. S. Sagaram, Y. E. Choi, J. So, H. H. Wilkinson \& Y. W. Lee, (2006) FSR1 is essential for virulence and female fertility in Fusarium verticillioides and F. graminearum. Mol Plant Microbe Interact 19: 725 733.

Simonin, A. R., C. G. Rasmussen, M. Yang \& N. L. Glass, (2010) Genes encoding a striatin-like protein (ham-3) and a forkhead associated protein (ham-4) are required for hyphal fusion in Neurospora crassa. Fungal Genet Biol 47: 855-868.

Smith, T. F., C. Gaitatzes, K. Saxena \& E. J. Neer, (1999) The WD repeat: a common architecture for diverse functions. Trends Biochem Sci 24: 181-185.

Sondek, J., A. Bohm, D. G. Lambright, H. E. Hamm \& P. B. Sigler, (1996) Crystal structure of a G-protein beta gamma dimer at 2.1A resolution. Nature 379: 369-374.

Song, Y., S. A. Cheon, K. E. Lee, S. Y. Lee, B. K. Lee, D. B. Oh, H. A. Kang \& J. Y. Kim, (2008) Role of the RAM network in cell polarity and hyphal morphogenesis in Candida albicans. Mol Biol Cell 19: 5456-5477.

Stegert, M. R., A. Hergovich, R. Tamaskovic, S. J. Bichsel \& B. A. Hemmings, (2005) Regulation of NDR protein kinase by hydrophobic motif phosphorylation mediated by the mammalian Ste20-like kinase MST3. Mol Cell Biol 25: 11019-11029.

Stegert, M. R., R. Tamaskovic, S. J. Bichsel, A. Hergovich \& B. A. Hemmings, (2004) Regulation of NDR2 protein kinase by multi-site phosphorylation and the S100B calcium-binding protein. J Biol Chem 279: 2380623812.

Stork, P. J. \& J. M. Schmitt, (2002) Crosstalk between cAMP and MAP kinase signaling in the regulation of cell proliferation. Trends Cell Biol 12: 258-266.

Sudbery, P. E. \& A. S. Gladfelter, (2008) Pathocycles. Fungal Genet Biol 45: 1-5.

Tamaskovic, R., S. J. Bichsel, H. Rogniaux, M. R. Stegert \& B. A. Hemmings, (2003) Mechanism of Ca2+-mediated regulation of NDR protein kinase through autophosphorylation and phosphorylation by an upstream kinase. J Biol Chem 278: 6710-6718. 
Towbin, H., T. Staehelin \& J. Gordon, (1979) Electrophoretic transfer of proteins from polyacrylamide gels to nitrocellulose sheets: procedure and some applications. Proc Natl Acad Sci U S A 76: 4350-4354.

Tucker, C. L., L. A. Peteya, A. M. Pittman \& J. Zhong, (2009) A genetic test for yeast two-hybrid bait competency using RanBPM. Genetics 182: 1377-1379.

Verde, F., D. J. Wiley \& P. Nurse, (1998) Fission yeast orb6, a ser/thr protein kinase related to mammalian rho kinase and myotonic dystrophy kinase, is required for maintenance of cell polarity and coordinates cell morphogenesis with the cell cycle. Proc Natl Acad Sci U S A 95: 7526-7531.

Vogel, H. J., (1956) A convenient growth medium for Neurospora (Medium N). . Microbial Genet. Bull. 13: 4243.

Vogel, H. J., (1964) Distribution of Lysine Pathways Among Fungi: Evolutionary Implications. The American Naturalist 98: 435-446.

Vogt, N. \& S. Seiler, (2008) The RHO1-specific GTPase-activating protein LRG1 regulates polar tip growth in parallel to Ndr kinase signaling in Neurospora. Mol Biol Cell 19: 4554-4569.

Walton, F. J., J. Heitman \& A. Idnurm, (2006) Conserved elements of the RAM signaling pathway establish cell polarity in the basidiomycete Cryptococcus neoformans in a divergent fashion from other fungi. Mol Biol Cell 17: 3768-3780.

Wang, C. L., W. B. Shim \& B. D. Shaw, (2010) Aspergillus nidulans striatin (StrA) mediates sexual development and localizes to the endoplasmic reticulum. Fungal Genet Biol 47: 789-799.

Wang, Y. \& H. G. Dohlman, (2004) Pheromone signaling mechanisms in yeast: a prototypical sex machine. Science 306: 1508-1509.

Wayman, G. A., Y. S. Lee, H. Tokumitsu, A. J. Silva \& T. R. Soderling, (2008) Calmodulin-kinases: modulators of neuronal development and plasticity. Neuron 59: 914-931.

Wei, H., N. Requena \& R. Fischer, (2003) The MAPKK kinase SteC regulates conidiophore morphology and is essential for heterokaryon formation and sexual development in the homothallic fungus Aspergillus nidulans. Mol Microbiol 47: 1577-1588.

Wei, X., T. Shimizu \& Z. C. Lai, (2007) Mob as tumor suppressor is activated by Hippo kinase for growth inhibition in Drosophila. EMBO J 26: 1772-1781.

Weiland, J. J., (1997) Rapid procedure for the extraction of DNA from fungal spores and mycelia. Fungal Genetics Newsletter 44: 60-63.

Weiss, E. L., C. Kurischko, C. Zhang, K. Shokat, D. G. Drubin \& F. C. Luca, (2002) The Saccharomyces cerevisiae Mob2p-Cbk1p kinase complex promotes polarized growth and acts with the mitotic exit network to facilitate daughter cell-specific localization of Ace2p transcription factor. J Cell Biol 158: 885-900.

Westfall, P. J., D. R. Ballon \& J. Thorner, (2004) When the stress of your environment makes you go HOG wild. Science 306: 1511-1512.

Whiteway, M. S., C. Wu, T. Leeuw, K. Clark, A. Fourest-Lieuvin, D. Y. Thomas \& E. Leberer, (1995) Association of the yeast pheromone response $\mathrm{G}$ protein beta gamma subunits with the MAP kinase scaffold Ste5p. Science 269: 1572-1575.

Whitmarsh, A. J. \& R. J. Davis, (1998) Structural organization of MAP-kinase signaling modules by scaffold proteins in yeast and mammals. Trends Biochem Sci 23: 481-485.

Williams, T. M. \& M. P. Lisanti, (2004a) The Caveolin genes: from cell biology to medicine. Ann Med 36: 584595.

Williams, T. M. \& M. P. Lisanti, (2004b) The caveolin proteins. Genome Biol 5: 214.

Winters, M. J., R. E. Lamson, H. Nakanishi, A. M. Neiman \& P. M. Pryciak, (2005) A membrane binding domain in the ste5 scaffold synergizes with gbetagamma binding to control localization and signaling in pheromone response. Mol Cell 20: 21-32.

Witczak, C. A., C. G. Sharoff \& L. J. Goodyear, (2008) AMP-activated protein kinase in skeletal muscle: from structure and localization to its role as a master regulator of cellular metabolism. Cell Mol Life Sci 65: 3737-3755.

Woodcock, D. M., P. J. Crowther, J. Doherty, S. Jefferson, E. DeCruz, M. Noyer-Weidner, S. S. Smith, M. Z. Michael \& M. W. Graham, , (1989) Quantitative evaluation of Escherichia coli host strains for tolerance to cytosine methylation in plasmid and phage recombinants. Nucleic Acids Res 17.

Xu, J. R., (2000) Map kinases in fungal pathogens. Fungal Genet Biol 31: 137-152.

Xu, J. R., C. J. Staiger \& J. E. Hamer, (1998) Inactivation of the mitogen-activated protein kinase Mps1 from the rice blast fungus prevents penetration of host cells but allows activation of plant defense responses. Proc Natl Acad Sci U S A 95: 12713-12718.

Xu, J. R., X. Zhao \& R. A. Dean, (2007) From genes to genomes: a new paradigm for studying fungal pathogenesis in Magnaporthe oryzae. Adv Genet 57: 175-218. 
Yablonski, D., I. Marbach \& A. Levitzki, (1996) Dimerization of Ste5, a mitogen-activated protein kinase cascade scaffold protein, is required for signal transduction. Proc Natl Acad Sci U S A 93: 13864-13869.

Yamamoto, T., Y. Moriwaki, S. Takahashi, Y. Nasako, J. Yamakita, K. Hiroishi \& K. Higashino, (1995) Determination of plasma purine nucleoside phosphorylase activity by high-performance liquid chromatography. Anal Biochem 227: 135-139.

Yamamura, Y. \& W. B. Shim, (2008) The coiled-coil protein-binding motif in Fusarium verticillioides Fsr1 is essential for maize stalk rot virulence. Microbiology 154: 1637-1645.

Yang, Y., P. Cheng \& Y. Liu, (2002) Regulation of the Neurospora circadian clock by casein kinase II. Genes Dev 16: 994-1006.

Yarden, O., M. Plamann, D. J. Ebbole \& C. Yanofsky, (1992) cot-1, a gene required for hyphal elongation in Neurospora crassa, encodes a protein kinase. EMBO J 11: 2159-2166.

Zallen, J. A., E. L. Peckol, D. M. Tobin \& C. I. Bargmann, (2000) Neuronal cell shape and neurite initiation are regulated by the Ndr kinase SAX-1, a member of the Orb6/COT-1/warts serine/threonine kinase family. Mol Biol Cell 11: 3177-3190.

Zeqiraj, E., B. M. Filippi, M. Deak, D. R. Alessi \& D. M. van Aalten, (2009a) Structure of the LKB1-STRAD-MO25 complex reveals an allosteric mechanism of kinase activation. Science 326: 1707-1711.

Zeqiraj, E., B. M. Filippi, S. Goldie, I. Navratilova, J. Boudeau, M. Deak, D. R. Alessi \& D. M. van Aalten, (2009b) ATP and MO25alpha regulate the conformational state of the STRADalpha pseudokinase and activation of the LKB1 tumour suppressor. PLoS Biol 7: e1000126.

Zhang, L., M. Bewick \& R. M. Lafrenie, (2002) Role of Raf-1 and FAK in cell density-dependent regulation of integrin-dependent activation of MAP kinase. Carcinogenesis 23: 1251-1258.

Zhao, Q., X. Wang, L. D. Nelin, Y. Yao, R. Matta, M. E. Manson, R. S. Baliga, X. Meng, C. V. Smith, J. A. Bauer, C. H. Chang \& Y. Liu, (2006) MAP kinase phosphatase 1 controls innate immune responses and suppresses endotoxic shock. J Exp Med 203: 131-140.

Ziv, C., G. Kra-Oz, R. Gorovits, S. Marz, S. Seiler \& O. Yarden, (2009) Cell elongation and branching are regulated by differential phosphorylation states of the nuclear Dbf2-related kinase COT1 in Neurospora crassa. Mol Microbiol 74: 974-989.

Zon, L. I., D. M. Dorfman \& S. H. Orkin, (1989) The polymerase chain reaction colony miniprep. Biotechniques 7: 696-698. 


\section{Acknowledgements}

Ich möchte mich zunächst bei den Mitgliedern meines Thesis Committees, PD Dr. Stephan Seiler, Prof. Dr. Stefanie Pöggeler und Prof. Dr. Reinhard Jahn, bedanken. Außerdem freue ich mich, dass Dr. Hans-Dieter Schmitt, Prof. Dr. Heike Krebber und Dr. Annette Borchers die Teilnahme als Mitglieder des Committees zur Dissertation übernommen haben. Vielen Dank dafür.

Darüberhinaus bedanke ich mich insbesondere bei Frau Prof. Dr. Stefanie Pöggeler für die Übernahme einer Referenz.

Ein ganz besonderer Dank gilt Stephan für die Möglichkeit in seinem Labor meine Doktorarbeit anzufertigen und für die sehr gute und kompetente fachliche Betreuung.

Ein sehr großer Dank gilt meinen lieben Laborkollegen Danni, Bine, Sarah, Matthias, Kerstin, Rösi, Immo und Corinna für das tolle Arbeitsklima während meiner Doktorarbeit. Wir sind durch viele Höhen und Tiefen gegangen, haben sehr viel erlebt, gelacht aber auch diskutiert und auch wegen euch bin ich jeden Tag mit sehr viel Freude ins Labor gegangen.

Vielen Dank auch an Flo und Yasmine, sowie allen Mitgliedern der Abteilung Braus und Pöggeler für das produktive Arbeitsklima.

Bei meinen Freunden Anika und Diana möchte ich mich ganz herzlich für die jahrelange Freundschaft auch über große Entfernungen bedanken.

Ein ganz ganz großer Dank geht an meine Eltern für die ideelle Unterstützung und Motivation während der letzten Jahre. Ohne euch wäre mein Studium und auch der Neubeginn in Göttingen nicht möglich gewesen. Vielen lieben Dank!

Ganz besonders liegt mir am Herzen mich bei Yvonne zu bedanken. Du hast mich immer unterstützt in beruflichen sowie privaten Dingen und mir auch mal den Kopf gewaschen. Du hast mich motiviert und vorangetrieben. Das Arbeiten im Labor wäre ohne dich nur halb so schön gewesen und das Schreiben der Doktorarbeit viel viel schwieriger. Danke für alles!!! 


\section{Curriculum vitae}

\section{Persönliche Daten}

Name:

Anne Dettmann

Anschrift:

Valentinsbreite 5, 37077 Göttingen

Geburtsdatum und -ort: $\quad$ 04.07.1981, Neubrandenburg

Staatsangehörigkeit: deutsch

\section{Schulbildung}

$1988-1994$

Grundschule, Brandenburg an der Havel

$1994-2001$

Märkisches Gymnasium „Friedrich Grasow“, Brandenburg an der Havel

06.2001

Allgemeine Hochschulreife

\section{Studium}

2001-2007

Ernst-Moritz-Arndt Universität Greifswald Studium der Biologie (Hauptfach: Genetik Nebenfächer: Biochemie, Mikrobiologie)

2007

Abschluss: Diplom-Biologin

Diplomarbeit mit dem Titel: „Histonmodifizierung und Regulation von Strukturgenen der Phospholipid-Biosynthese in der Hefe Saccharomyces cerevisiae"

Promotion an der Georg-August Universität Göttingen im Rahmen des Promotionsprogramms "Molecular Biology of Microbial, Animal and Plant Cells" der Göttinger Graduate School for Neurosciences and Molecular Biosciences (GGNB)

2011

Doktorarbeit mit dem Titel: „Regulation and communication between the NDR kinase COT1, the MAK2 MAP kinase and the Striatin complex in Neurospora crassa" 